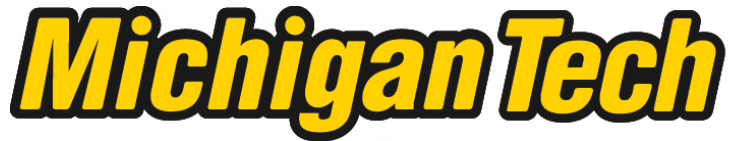 \\ Michigan Technological University Create the Future Digital Commons @ Michigan Tech
}

Dissertations, Master's Theses and Master's Reports - Open

Dissertations, Master's Theses and Master's

Reports

2009

Controlling electronic and magnetic properties of ultra narrow multilayered nanowires

Puspamitra Panigrahi

Michigan Technological University

Follow this and additional works at: https://digitalcommons.mtu.edu/etds

Part of the Physics Commons

Copyright 2009 Puspamitra Panigrahi

\section{Recommended Citation}

Panigrahi, Puspamitra, "Controlling electronic and magnetic properties of ultra narrow multilayered nanowires", Dissertation, Michigan Technological University, 2009.

https://doi.org/10.37099/mtu.dc.etds/116

Follow this and additional works at: https://digitalcommons.mtu.edu/etds

Part of the Physics Commons 
CONTROLLING ELECTRONIC AND MAGNETIC PROPERTIES OF ULTRA NARROW MULTILAYERED NANOWIRES

By

\title{
PUSPAMITRA PANIGRAHI
}

\author{
A DISSERTATION \\ Submitted in partial fulfillment of the requirements \\ for the degree of \\ DOCTOR OF PHILOSOPHY \\ (Physics) \\ MICHIGAN TECHNOLOGICAL UNIVERSITY \\ 2009
}

(C) 2009 Puspamitra Panigrahi 

This dissertation, "Controlling electronic and magnetic properties of ultra narrow multilayered nanowires", is hereby approved in partial fulfillment of the requirements for the degree of DOCTOR OF PHILOSOPHY in the field of Physics.

DEPARTMENT:

Physics

Signatures:

Dissertation Advisor

Dr. Ranjit Pati

Committee

Dr. Max Seel

Dr. Ravi Pandey

Dr. G.M.Odegard

Department Chair

Dr. Ravi Pandey

Date 



\section{Dedication}

Dedicated to my bettu Aaryan 


\section{Contents}

List of Figures

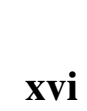

List of Tables

xviii

Acknowledgments

$\underline{\text { xix }}$

Abstract

$\underline{\mathbf{x x i}}$

1 Introduction

1

1.1 Review of Current Understanding of Magnetic/non-magnetic Heterostruc-

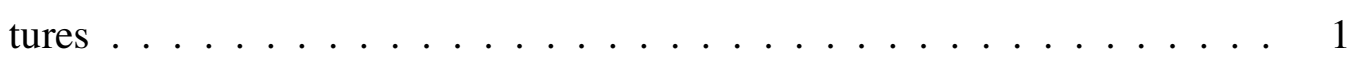

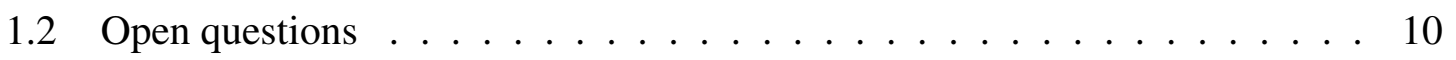


2.1 Introduction ......................... 13

2.2 Schrödinger Equation ............................. 14

2.3 Born-Oppenheimer Approximation .................... 15

2.4 Electron-electron interaction $\ldots \ldots \ldots \ldots \ldots$

2.4.1 Hartree-Fock Theory .................. 16

2.4.2 Correlation problem in Hartree-Fock approach .......

2.4 .3 Density Functional Theory . . . . . . . . . . . . . 19

2.4.3.1 Thomas-Fermi Model . . . . . . . . . . . . . 19

2.4.3.2 Hohenberg-Kohn theorems ..........

2.4.3.3 The Levy Constrained search .......... . .

2.4.3.4 The Kohn-Sham Formulations ......... . . 
2.5 Supercell approach

2.5.1 Bloch's theorem .................... 40

2.5.2 Brillouin zone sampling ................... 41

2.5.3 Plane-wave basis sets ......................... 44

2.5.4 Plane-wave picture of Kohn-Sham equations . . . . . . . . . 44

2.6 Pseudopotential Approximation $\ldots \ldots \ldots \ldots$

2.7 Computational Procedure for calculation of ground state charge density and

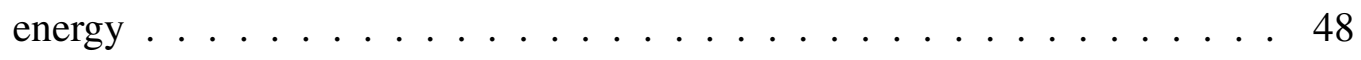

3 Tuning ferromagnetism in multilayered one dimensional barcode nanowires 51

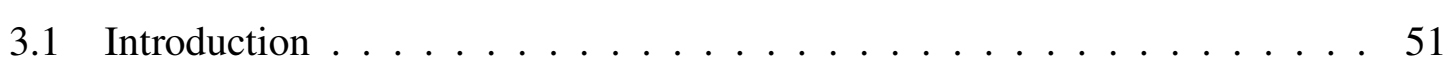

3.2 Computational approach $\ldots \ldots \ldots \ldots \ldots \ldots \ldots \ldots$

3.3 Results and discussions .......................... 57

3.3.1 Structure and stability ...................... 58 
3.3.2 Magnetic Properties ...................... 60

3.3.3 Spin polarized density of states .............. 68

3.3.4 Spin polarized energy bands .................. 71

3.3.5 Comparison with $\mathrm{Ni} / \mathrm{Cu}$ and $\mathrm{Ni} / \mathrm{Al}$ System ............. 75

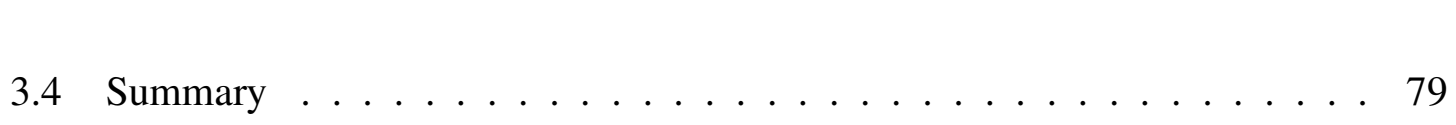

4 Controlling Interlayer Exchange Coupling in one dimensional Fe/Pt Multilayered nanowire

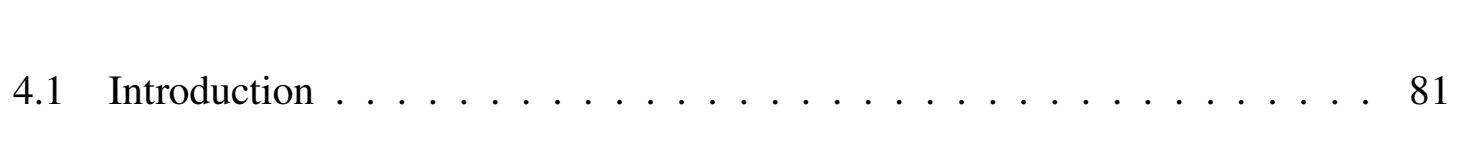

4.2 Theoretical Approach ........................... 83

4.3 Results and discussion $\ldots \ldots \ldots \ldots$

4.3.1 Stability and magnetic properties ............. 86

4.3.2 Switching of IEC with Platinum spacer . . . . . . . . . . 89

4.3.3 Spin polarized energy bands .....................

4.3 .4 Conductance ................................. 


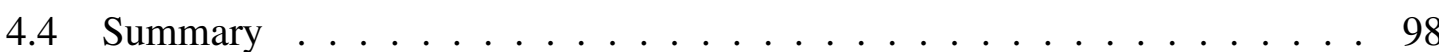

5 Role of spacer on Interlayer Exchange coupling in multilayered nanowire 99

5.1 Introduction . . . . . . . . . . . . . . . . . 99

5.2 Theoretical Approach . . . . . . . . . . . . . . . . 101

5.3 Results and Discussion . . . . . . . . . . . . . . . 102

5.3.1 Structure stability and magnetic properties . . . . . . . . . 102

5.3.2 The role of spacers on IEC . . . . . . . . . . . . . . 104

5.3.2.1 Fe/Pd Nanowire . . . . . . . . . . . . . 106

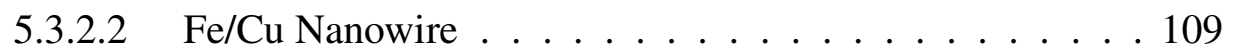

5.3.2.3 Fe/Ag and Fe/Au Multilayered Nanowires . . . . . . . 112

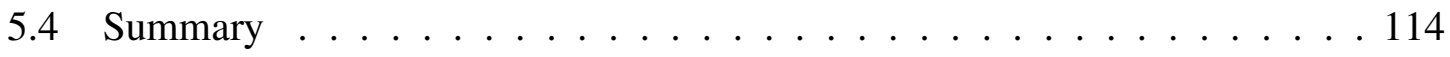

6 General Conclusions, Perspectives and Future Directions 117

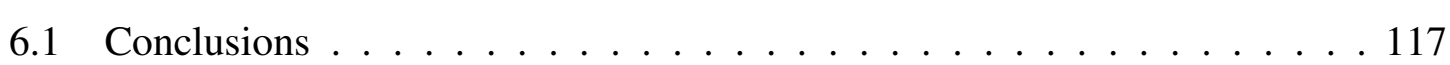


6.2 Future directions: . . . . . . . . . . . . . . . . . . 120

A Derivation of Thomas Fermi kinetic energy

B N representability and V representability

C Time Line

D Copyrights

Bibliography

135 


\section{List of Figures}

1.1 RKKY interaction: the interaction of magnetic spins mediated by conduction electrons. . . . . . . . . . . . . . . . . . .

1.2 GMR effect in $\mathrm{Fe} / \mathrm{Cr} / \mathrm{Fe}$ multilayered structure. (accessed on March 20, 2009;

Copyright -Guillom, Wikimedia commons, 2007, distributed under GFDL, refer Appendix $\mathrm{C}$ for permission) $\ldots \ldots \ldots \ldots$

1.3 Schematic representation of spin valve GMR effect(accessed on March 21, 2009; Copyright -Guillom, Wikimedia commons, 2006, distributed under GFDL, refer Appendix $\mathrm{C}$ for permission) $\quad \ldots \ldots \ldots \ldots$

2.1 A schematic view of true ionic potential and valance wave function with the corresponding pseudopotential and pseudo-wave function(accessed on January 10, 2009; Copyright - Wquester, Wikipedia, 2006, distributed under public domain, refer Appendix C for permission.) . . . . . . . . . . 47 
2.2 Flow chart for the computational approach . . . . . . . . . . . 49

3.1 ABCABCABC stacking of the fcc Fe; (a) side view, (b) top view used to construct the Fe-nanowire . . . . . . . . . . . . . . . . 54

3.2 Calculated cohesive energy, $E_{c}$,(black line), and magnetic moment(blue line) per Iron atom as a function of lattice parameter in fcc solid, obtained using LSDA approximation . . . . . . . . . . . . . . . . 55

3.3 Calculated cohesive energy, $E_{c},($ black line), and magnetic moment(blue line) per Iron atom as a function of lattice parameter in fcc solid, obtained using GGA approximation . . . . . . . . . . . . . . . . . . 56

3.4 Calculated cohesive energy, $E_{c}$, per Platinum atom as a function of lattice parameter in fcc solid, obtained using LSDA approximation . . . . . . . . . 57

3.5 Optimized(a) Fe39; (b) Fe36Pt3; (c) Fe23Pt16; and (d) Fe10Pt29-nanowire structures. Notation: golden yellow (Fe); silver white (Pt). Two unit cells for each structure are presented for better visualization. Reprinted figure with permission from, Puspamitra.Panigrahi, Ranjit.Pati, Phys. Rev. B. 76, 024431(2007), (c)(2007) The American Physical society. . . . . . . . . . 59 
3.6 Calculated cohesive energy, Ec, as a function of the number of Pt spacer layers, $N(P t)$, in the nanowire structures. Reprinted figure with permission from Puspamitra.Panigrahi, Ranjit.Pati, Phys. Rev. B. 76, 024431(2007), (c)(2007) The American Physical society . . . . . . . . . . . . . . . 60

3.7 Calculated magnetic moment per Fe atom $\left(\mu_{a v}\right)$ as a function of the number of Pt spacer layers, $N(P t)$. Reprinted figure with permission from Puspamitra.Panigrahi, Ranjit.Pati, Phys. Rev. B. 76, 024431(2007), (C)(2007) The American Physical society . . . . . . . . . . . . . . 61

3.8 Schematic representation of spin flipping and multistep electron transfer process in $\mathrm{Fe}_{23} \mathrm{Pt}_{16}$ barcode wire . . . . . . . . . . . . 65

3.9 Inverse of number of Fe layers $N(F e)$, in the multilayer as a function of the number of Pt spacer layers, $N(P t) \ldots \ldots \ldots 7$

3.10 Equilibrium $\mathrm{Fe}_{43}$-nanowire structure of $\approx 1.1 \mathrm{~nm}$ diameter.6unit cells are presented for clarity. ....................... 68

3.11 Projected spin-polarized d-band density of states (PDOS) of Fe in $\mathrm{Fe}_{39}$ barcode nanowires. Reprinted figure with permission from, Puspamitra.Panigrahi, Ranjit.Pati, Phys. Rev. B. 76, 024431(2007), (C(2007) The American Physical society. . . . . . . . . . . . . . . . . . . . . 69 
3.12 Projected spin-polarized d-band density of states (PDOS) of Fe in Fe ${ }_{23} \mathrm{Pt}_{16}$ barcode nanowires. Reprinted figure with permission from, Puspamitra.Panigrahi, Ranjit.Pati, Phys. Rev. B. 76, 024431(2007), (c)(2007) The American Physical society. . . . . . . . . . . . . . . . . . . . 70

3.13 Projected spin-polarized d-band density of states (PDOS) of Pt in $\mathrm{Fe}_{23} \mathrm{Pt}_{16}$ barcode nanowires. Reprinted figure with permission from, Puspamitra.Panigrahi, Ranjit.Pati, Phys. Rev. B. 76, 024431(2007), (C)(2007) The American Physical society. . . . . . . . . . . . . . . . .

3.14 Spin polarized electronic band structures for (i)Fe 39 (spin up); (ii) $\mathrm{Fe}_{39}$ (spin down). Reprinted figure with permission from, Puspamitra.Panigrahi, Ranjit.Pati, Phys. Rev. B. 76, 024431(2007), (c)(2007) The American Phys-

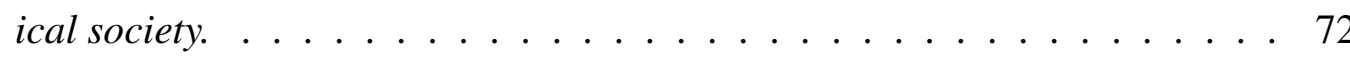

3.15 Spin polarized electronic band structures for (i) $\mathrm{Fe}_{36} \mathrm{Pt}_{3}$ (spin up); (ii)Fe $\mathrm{F}_{39} \mathrm{Pt}_{3}$ (spin down).Reprinted figure with permission from, Puspamitra.Panigrahi, Ranjit.Pati, Phys. Rev. B. 76, 024431(2007), (C)(2007) The American Physical society. . . . . . . . . . . . . . . . . 73 
3.16 Spin polarized electronic band structures for (i) $\mathrm{Fe}_{23} \mathrm{Pt}_{16}$ ( (spin up); (ii)Fe $\mathrm{e}_{23} P t_{16}$ (spin down). Reprinted figure with permission from, Puspamitra.Panigrahi, Ranjit.Pati, Phys. Rev. B. 76, 024431(2007), (C(2007) The American Physical society. . . . . . . . . . . . . . . . . . . 74

3.17 Spin polarized electronic band structures for (i) $\mathrm{Fe}_{10} P t_{29}$ ( spin up); ( $i$ i) $\mathrm{Fe}_{10} \mathrm{Pt}_{29}$ (spin down). Reprinted figure with permission from, Puspamitra.Panigrahi, Ranjit.Pati, Phys. Rev. B. 76, 024431(2007), (C)(2007) The American Physical society. .............................. 75

3.18 Calculated cohesive energy $E_{c}$ as a function of Number of $C u$ layers $\left(N_{C u}\right)$ in $\mathrm{Ni} / \mathrm{Cu}$ multilayered nanowire(Reprinted figure with permission from, Partha.Pratim.Pal, Ranjit.Pati, Phys. Rev. B. 77, 144430(2008), (C)(2008) The American Physical society: refer Appendix C for permission) . . . . 77

3.19 Calculated cohesive energy $E_{c}$ as a function of Number of Al layers $\left(N_{A l}\right)$ in Ni/Al multilayered nanowire(Reprinted figure with permission from, Partha.Pratim.Pal, Ranjit.Pati, Phys. Rev. B. 77, 144430(2008), (C)(2008) The American Physical society: refer Appendix C for permission) . . . . 77 
3.20 Calculated magnetic moment per Ni atom $\left(\mu_{a v}\right)$ as a function of number of Al layers $\left(N_{A l}\right)$ in $\mathrm{Ni} / \mathrm{Al}$ multilayered nanowire.(Reprinted figure with permission from, Partha.Pratim.Pal, Ranjit.Pati, Phys. Rev. B. 77, 144430(2008), (C)(2008) The American Physical society: refer Appendix C for permission $) \ldots \ldots \ldots \ldots \ldots \ldots \ldots \ldots$

3.21 Calculated magnetic moment per Ni atom $\left(\mu_{a v}\right)$ as a function of Number of $\mathrm{Cu}$ layers $\left(\mathrm{N}_{\mathrm{Cu}}\right)$ in $\mathrm{Ni} / \mathrm{Cu}$ multilayered nanowire(Reprinted figure with permission from, Partha.Pratim.Pal, Ranjit.Pati, Phys. Rev. B. 77, 144430(2008), (c)(2008) The American Physical society: refer Appendix $\mathrm{C}$ for permission $) \ldots \ldots \ldots \ldots \ldots \ldots \ldots$

4.1 Optimized structures for the Fe/Pt nanowire with two Pt spacer, five Pt spacer, and eight Pt spacer layers respectively. Notations: dark gray (red), Fe; light gray (yellow), Pt. Reprinted figure with permission from, Puspamitra.Panigrahi, Ranjit.Pati, Phys. Rev. B. 79, 014411(2009), (C)(2009) The American Physical society. . . . . . . . . . . . . . . . . . . . 87 
4.2 Calculated inter layer exchange coupling ( $J$ ), as a function of number of non-magnetic Pt spacer layers $N(P t)$ in the nanowire structures. $1 \times 1 \times$ 1 and $1 \times 1 \times 5$ represent the k-point sampling of the BZ used to obtain the respective results. Reprinted figure with permission from, Puspamitra.Panigrahi, Ranjit.Pati, Phys. Rev. B. 79, 014411(2009), (C)(2009) The American Physical society. . . . . . . . . . . . . . . . . 89

4.3 Histogram plot for the average magnetic moment per atom $\left(\mu_{a v}\right)$ in ferromagnetic $(F M)$ and anti-ferromagnetic $(A F M)$ configurations for two Pt spacer. Reprinted figure with permission from, Puspamitra.Panigrahi, Ranjit.Pati, Phys. Rev. B. 79, 014411(2009), (C)(2009) The American Physical

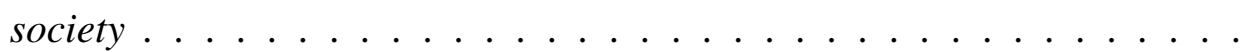

4.4 Histogram plot for the average magnetic moment per atom $\left(\mu_{a v}\right)$ in ferromagnetic (FM) and anti-ferromagnetic (AFM) configurations for five Pt spacer. Reprinted figure with permission from, Puspamitra.Panigrahi, Ranjit.Pati, Phys. Rev. B. 79, 014411(2009), (C)(2009) The American Physical

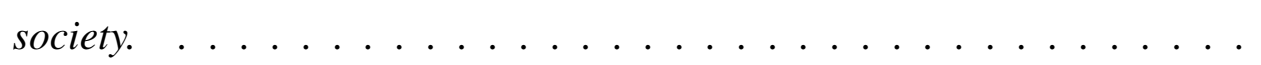


4.5 Histogram plot for the average magnetic moment per atom $\left(\mu_{a v}\right)$ in ferromagnetic (FM) and anti-ferromagnetic (AFM) configurations for eight Pt spacer. Reprinted figure with permission from, Puspamitra.Panigrahi, Ranjit.Pati, Phys. Rev. B. 79, 014411(2009), (c)(2009) The American Physical

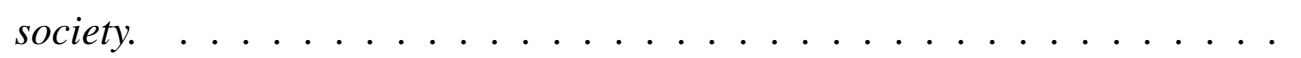

4.6 Spin polarized energy band structures for 2-spacer system.The Fermi energy is set at $E=0$. Notations: gray (red) circle, conduction band; gray (blue) diamond, valence band.Reprinted figure with permission from, Puspamitra.Panigrahi, Ranjit.Pati, Phys. Rev. B. 79, 014411(2009), (C)(2009) The American Physical society. . . . . . . . . . . . . . . . . . . . .

4.7 Spin polarized energy band structures for 5-spacer system.The Fermi energy is set at $E=0$. Notations: gray (red) circle, conduction band; gray (blue) diamond, valence band. Reprinted figure with permission from, Puspamitra.Panigrahi, Ranjit.Pati, Phys. Rev. B. 79, 014411(2009), (C)(2009) The American Physical society. . . . . . . . . . . . . . . . . . . . .

4.8 Spin polarized energy band structures for 8-spacer system.The Fermi energy is set at $E=0$. Notations: gray (red) circle, conduction band; gray (blue) diamond, valence band. Reprinted figure with permission from, Puspamitra.Panigrahi, Ranjit.Pati, Phys. Rev. B. 79, 014411(2009), (C)(2009) The American Physical society. . . . . . . . . . . . . . . . . . . . . 96 
5.1 Optimized structures for the Fe/spacer nanowire with two spacer, and five spacer layers respectively in the FM and AFM configuration. The top two wires correspond to the structure in FM configuration; the bottom two figures correspond to the structure in AFM configuration. Notations: dark gray (red), Fe; light gray (green and blue), spacers. Spacer atoms: $P d$, $C u, A u, A g$

5.2 Spin polarized energy band structures for 2-spacer Fe/Pd system.The Fermi energy is set at $E=0$. Notations: gray (blue) diamond, conduction band; gray $($ red $)$ star, valence band. . . . . . . . . . . . . . . . . . . 107

5.3 Spin polarized energy band structures for 5-spacer Fe/Pd system.The Fermi energy is set at $E=0$. Notations: gray (blue) diamond, conduction band; gray $($ red) star, valence band. . . . . . . . . . . . . . . . . . 108

5.4 Spin polarized energy band structures for 2-spacer Fe/Cu system.The Fermi energy is set at $E=0$. Notations: gray (blue) diamond, conduction band; gray $($ red $)$ star, valence band. . . . . . . . . . . . . . . . . . 110

5.5 Spin polarized energy band structures for 5-spacer Fe/Cu system. The Fermi energy is set at $E=0$. Notations: gray (blue) diamond, conduction band; gray $($ red $)$ star, valence band. . . . . . . . . . . . . . . . . . 111 
5.6 Spin polarized energy band structures for 2-spacer Fe/Ag system.The Fermi energy is set at $E=0$. Notations: gray (blue) diamond, conduction band; gray $($ red $)$ star, valence band. . . . . . . . . . . . . . . . . . 113

5.7 Spin polarized energy band structures for 2-spacer Fe/Au system.The Fermi energy is set at $E=0$. Notations: gray (blue) diamond, conduction band; gray $($ red $)$ star, valence band. . . . . . . . . . . . . . . . . . 114

2.1 A mapping between potential and ground state density. Though the local potential can have multiple ground states, the two different local potential will never give the same ground state density. . . . . . . . . . . . 128

4.1 The photo copy of the permission letter showing permission is granted for the figures.(Reprinted from, Partha.Pratim.Pal, Ranjit.Pati, Phys. Rev. B. 77, 144430(2008), (c)(2008) The American Physical society.) . . . . . . . . 133 


\section{List of Tables}

3.1 Comparison of individual magnetic moment (in the unit of $\mu_{B}$ ) of $F e$ atoms in $\mathrm{Fe}_{39}$ (pristine)nanowire with that of the corresponding $\mathrm{Fe}$ atoms in $\mathrm{Fe}_{36} \mathrm{Pt}_{3}$ (1-Layer Pt), $\mathrm{Fe}_{23} P t_{16}$ (4-Layer Pt), $F e_{10} P t_{29}$ (7-Layer Pt) multilayered

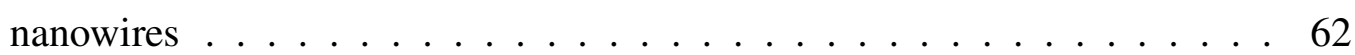

4.1 Comparison of individual magnetic moment (unit $\mu_{B}$ of $F e$ atom in 2spacer,5-spacer,8-spacer layer multilayered nanowire systems in both FM and AFM configuration with that of corresponding $F e$ atoms in the pristine

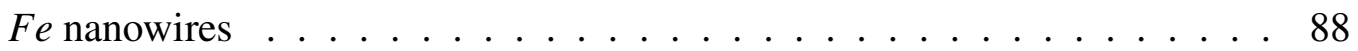

4.2 Calculated Polarization, Conductance, and GMR in the nanowire for different spacer lengths. . . . . . . . . . . . . . . . 97 
5.1 Results for exchange coupling energy $J$ in 2-spacer, and 5-spacer multilayered nanowire systems obtained with $(1 \times 1 \times 1)$ k-point sampling of the Brillouin zone. . . . . . . . . . . . . . . . . . . . 104

5.2 Results for exchange coupling energy $J$ in 2-spacer,and 5-spacer multilayered nanowire systems obtained with $(1 \times 1 \times 5)$ k-point sampling of the Brillouin zone. . . . . . . . . . . . . . . . . . 105

5.3 Comparison of individual average magnetic moment (unit $\mu_{B}$ associated with soacer layers in 2-spacer multilayered nanowire systems. . . . . . . 105

3.1 comparison between different k-point sampling. . . . . . . . . . . . 129 


\section{Acknowledgments}

This part of my project arose out of years of research initiated at MTU,US. I have worked with great number of people whose contribution in assorted way for my research and life deserved special mention and it is my pleasure to convey this.

In the first place I would like to express my deep and sincere gratitude to my advisor Dr.Ranjit Pati for his wholehearted help, his enthusiasm and inspiration. I wish to express warm and sincere thanks to Dr. Ravindra Pandey for his unflinching encouragement and support in many ways.

My sincere thanks are due to Dr. Max Seel and Dr.G.M.Odegard for their detailed reviews, constructive criticism and excellent advice.

During my work I have discussed with colleagues Partha, Pavan, Subhasis, Brandon, Mike who made Physics fun for me and for whom I have deep regards.

I would like to thank CEC and Physics department, all the teachers and staff for providing me this space and opportunity for carrying out my research. Special thanks to Gowtham and Lin Pan for helping my writing my thesis in Letax.

I am deeply indebted to my parents and parent-in-laws for their patient love and faith on me. Their ideals and values have remarkable influence on me. I wish to thank my entire family 
including my sister Sanghamitra and niece Shweta for providing me a loving environment.

I owe my loving thanks to my son Aaryan and my husband Akshaya for their encouragement, understanding and sacrifice. I would appreciate them for their dedication and persistent confidence on me and let their enthusiasm, intelligence and ambition unselfishly collide with me. 


\section{Abstract}

Interest in the study of magnetic/non-magnetic multilayered structures took a giant leap since Grüberg and his group established that the interlayer exchange coupling (IEC) is a function of the non-magnetic spacer width. This interest was further fuelled by the discovery of the phenomenal Giant Magnetoresistance (GMR) effect. In fact, in 2007 Albert Fert and Peter Grünberg were awarded the Nobel Prize in Physics for their contribution to the discovery of GMR. GMR is the key property that is being used in the read-head of the present day computer hard drive as it requires a high sensitivity in the detection of magnetic field. The recent increase in demand for device miniaturization encouraged researchers to look for GMR in nanoscale multilayered structures. In this context, one dimensional (1-D) multilayerd nanowire structure has shown tremendous promise as a viable candidate for ultra sensitive read head sensors. In fact, the phenomenal giant magnetoresistance (GMR) effect, which is the novel feature of the currently used multilayered thin film, has already been observed in multilayered nanowire systems at ambient temperature. Geometrical confinement of the supper lattice along the 2-dimensions (2-D) to construct the 1-D multilayered nanowire prohibits the minimization of magnetic interaction- offering a rich variety of magnetic properties in nanowire that can be exploited for novel functionality. In addition, introduction of non-magnetic spacer between the magnetic layers presents additional advantage in controlling magnetic properties via tuning the interlayer magnetic interaction. Despite of a large volume of theoretical works devoted towards the under- 
standing of GMR and IEC in super lattice structures, limited theoretical calculations are reported in 1-D multilayered systems. Thus to gauge their potential application in new generation magneto-electronic devices, in this thesis, I have discussed the usage of first principles density functional theory (DFT) in predicting the equilibrium structure, stability as well as electronic and magnetic properties of one dimensional multilayered nanowires. Particularly, I have focused on the electronic and magnetic properties of Fe/Pt multilayered nanowire structures and the role of non-magnetic Pt spacer in modulating the magnetic properties of the wire. It is found that the average magnetic moment per atom in the nanowire increases monotonically with an $\sim \frac{1}{N(F e)}$ dependance, where $N(F e)$ is the number of iron layers in the nanowire. A simple model based upon the interfacial structure is given to explain the $\frac{1}{N(F e)}$ trend in magnetic moment obtained from the first principle calculations. A new mechanism, based upon spin flip with in the layer and multistep electron transfer between the layers, is proposed to elucidate the enhancement of magnetic moment of Iron atom at the Platinum interface. The calculated IEC in the $F e / P t$ multilayered nanowire is found to switch sign as the width of the non-magnetic spacer varies. The competition among short and long range direct exchange and the super exchange has been found to play a key role for the non-monotonous sign in IEC depending upon the width of the Platinum spacer layer. The calculated magnetoresistance from Julliere's model also exhibit similar switching behavior as that of IEC. The universality of the behavior of exchange coupling has also been looked into by introducing different non-magnetic spacers like Palladium, Copper, Silver, and Gold in between magnetic Iron layers. The nature of 
hybridization between $\mathrm{Fe}$ and other non-magnetic spacer is found to dictate the inter layer magnetic interaction. For example, in $F e / P d$ nanowire the d-p hybridization in two spacer layer case favors anti-ferromagnetic (AFM) configuration over ferromagnetic (FM) configuration. However, the hybridization between half-filled $\mathrm{Fe}(d)$ and filled $\mathrm{Cu}(\mathrm{p})$ state in $\mathrm{Fe} / \mathrm{Cu}$ nanowire favors FM coupling in the 2-spacer system. 


\section{Chapter 1}

\section{Introduction}

\subsection{Review of Current Understanding of Magnetic/non- magnetic Heterostructures}

Over the past years, the intense research focus on the magnetic and electronic properties of magnetic multilayered structures for their multifunctional properties has lead to successful applications of these heterostructures in a wide range of magneto-electronic devices such as magnetic sensors and ultrahigh density memory devices. Research on the magnetic and nonmagnetic heterostructure goes back to as early as 1986, when Grünberg et al.[1] established for the first time the importance of interlayer exchange coupling (IEC) in $\mathrm{Fe} / \mathrm{Cr} / \mathrm{Fe}$ layered structures. At zero magnetic field, it was shown that when a non-magnetic $\mathrm{Cr}$ 
layer of suitable thickness was sandwiched between a pair of magnetic $\mathrm{Fe}$ layers, the antiferromagnetic(AF) coupling between the Fe layers favored over the ferromagnetic (FM) coupling. The exchange interaction between the magnetic $F e$ layers mediated by the conduction electrons of the non-magnetic spacer was found to stabilize the AF configuration by lowering the total energy of the system, which can be understood from RudermanKittel-Kasuya-Yosida(RKKY) interaction mechanism. The RKKY mechanism describes the coupling of nuclear magnetic moments or localized inner $d$ or $f$ shell electron spins in a metal via conduction electrons. Depending upon the separation between a pair of ions their magnetic coupling can be ferromagnetic or antiferromagnetic. A magnetic ion induces a spin polarization in the conduction electrons in its neighborhood. This spin polarization in the itinerant electrons is felt by the moments of other magnetic ions within range, leading to an indirect coupling.

The interaction between two magnetic impurities arise when they are close enough to have appreciable overlap. If a second impurity is within the spin-down region of the first impurity's conduction electron polarization, it is favored to point oppositely to the polarization, and thus ferromagnetically with the first impurity. If the second impurity is further away, in the ring of spin-up (away from 1st ring of spin down), the second impurity prefers to point down, thus antiferromagnetic coupling with the first impurity. Thus, there is an interaction between two impurities induced by the spin-polarized conduction electrons with which they interact. The interaction takes the same distance dependence as the spin polarization, but is of opposite sign as shown in Figure. 1.1 


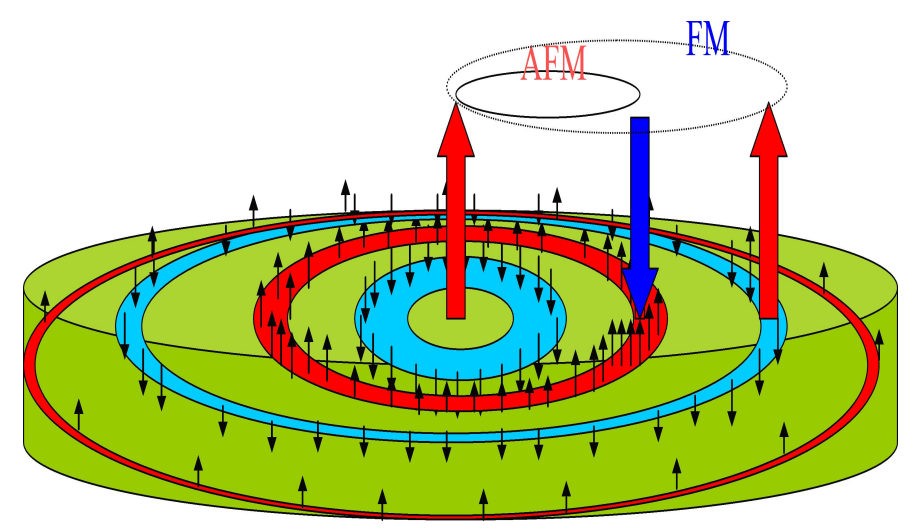

Figure 1.1: RKKY interaction: the interaction of magnetic spins mediated by conduction electrons.

The RKKY exchange coefficient, $J$, oscillates from positive to negative as the separation of the ions changes. It has the damped oscillatory nature. Subsequently, the discovery of giant magneto resistive (GMR)effect[2, 3] in 1988 in a magnetic multilayered structure revolutionized the interest in multilayered structures. In multilayer GMR, two or more ferromagnetic layers are separated by a very thin (about $1 \mathrm{~nm}$ ) non-ferromagnetic spacer (e.g. $\mathrm{Fe} / \mathrm{Cr} / \mathrm{Fe}$ ). The electrical resistance of a device is normally higher is the anti-parallel case. When the current is allowed to pass through a $\mathrm{Fe} / \mathrm{Cr} / \mathrm{Fe}$ layered structure, which favors $\mathrm{AF}$ configuration at zero field, maximum resistance to the flow of electrons in encountered. But when the applied magnetic field is increased, the spin of two magnetic layers reorient with respect to each other giving a low resistance path to the flow of electron until it reaches the saturation point. At a saturated applied magnetic field, $H_{s}$, the spin orientation of the two magnetic layers reorient themselves to FM configurations giving a least resistance path to the flow of current. A significant $80 \%$ change in resistance (10\% at room temperature) was found between FM and AFM configurations as shown in Figure. 1.2 


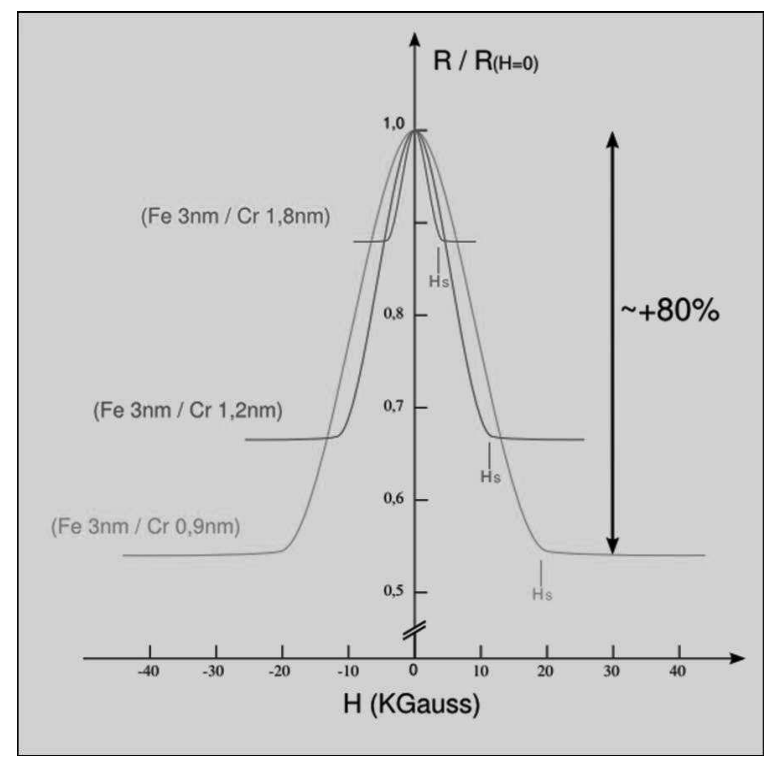

Figure 1.2: GMR effect in $\mathrm{Fe} / \mathrm{Cr} / \mathrm{Fe}$ multilayered structure. (accessed on March 20, 2009; Copyright -Guillom, Wikimedia commons, 2007, distributed under GFDL, refer Appendix $\mathrm{C}$ for permission)

This huge change in resistance is commonly refereed as GMR effect or spin valve effect, which can be understood as follows. In a circuit, electrical resistance arises due to scattering of electrons within the wire material. Depending upon the magnetic moment orientation, a single domain magnetic material will scatter "up" and "down" spin electrons differently. When the ferromagnetic layers in GMR structures are aligned anti-parallel, the resistance is high because the "spin-up" electrons that are not scattered in one layer (low resistance) can be scattered in the other layer (higher resistance). When the layers are aligned in parallel, all the "spin-up" electrons will not scatter much (low resistance), regardless of which ferromagnetic layer they pass through. This spin-dependent scattering phenomena which given rise to GMR is illustrated in Fig. 1.3 


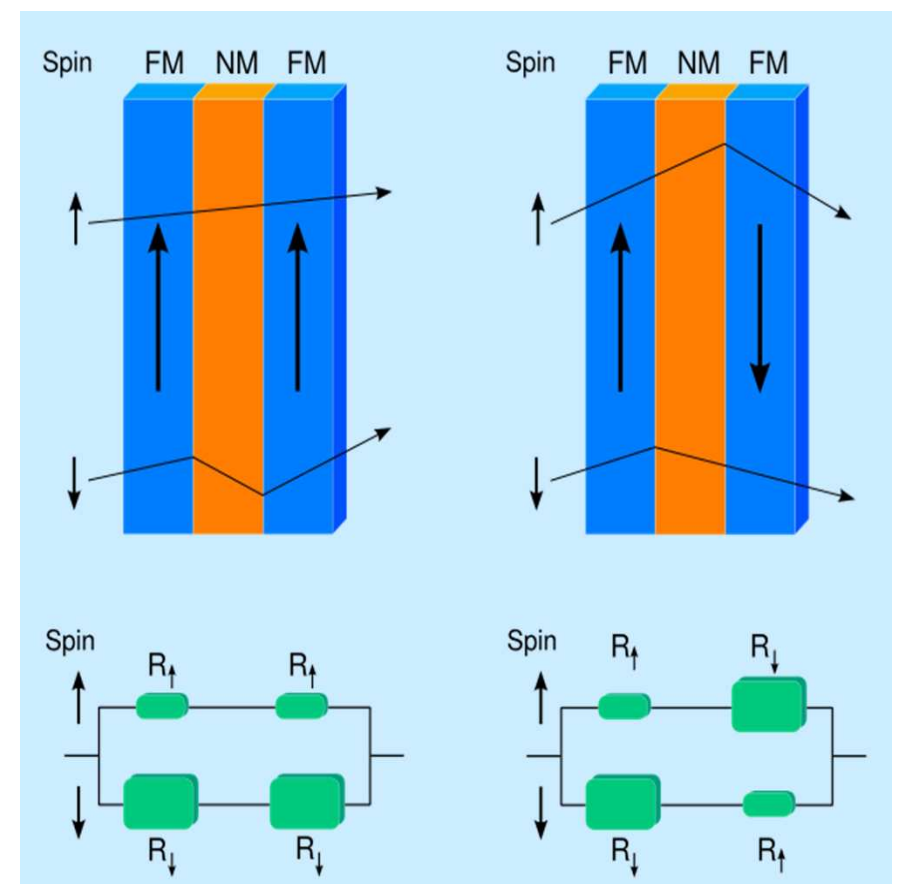

Figure 1.3: Schematic representation of spin valve GMR effect (accessed on March 21, 2009; Copyright -Guillom , Wikimedia commons, 2006, distributed under GFDL, refer Appendix C for permission)

The GMR effect and plays the key role in the read head sensor of modern hard drive disk and other high sensitive magneto-electronic devices. Peter Grünberg and Albert Fert shared recently (year 2007) the Nobel Prize in Physics for the discovery of this phenomenal GMR effect. Following the discovery of the GMR effect, interesting phenomena such as long

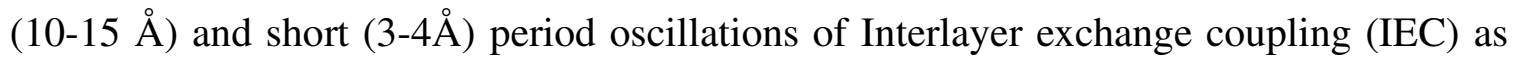
well as the oscillation of GMR were reported [4, 5, 6] in $\mathrm{Fe} / \mathrm{Cr}$ layered structure. This result was in contrast to the previously observed monotonically decreasing trend [2, 3] of GMR with spacer layer thickness. The oscillation was not observed earlier as complete control over the spacer thickness was not achieved. The magnitude of saturation field was also found to oscillate with the same period as that of magneto resistance and thus 
explained the origin of IEC. The oscillation in GMR is caused by the alteration in the sign of the interlayer exchange coupling between ferromagnetic layers. Moreover, the exchange coupling is found to oscillate between ferromagnetic and anti-ferromagnetic depending upon the magnetic moment of successive ferromagnetic layers. Taking $\mathrm{Cu}-\mathrm{Co}$ as an example, the largest value of GMR of $110 \%$ requires magnetic field of 20 KOe, whereas $50-60 \%$ of the GMR can be achieved with few hundred Oe field range which further drops down to field of few ten Oe to obtain $20 \%$ of the GMR. This controlled variation of GMR is achievable by varying the IEC between the $C o$ layers with different $C u$ layer thickness.

Similar oscillations in GMR and IEC were also found when transition metal including $P t$ and Nobel metals like $C u, A u$ were used as spacers in magnetic/non-magnetic multilayered structures. This observed oscillatory behavior of magnetoresistance and IEC with the increase in non-magnetic spacer layer thickness in different superlattice structures suggests that this is a universal feature in magnetic and non-magnetic heterostructures. Such varying magnetoresistance values at room temperature makes the bulk multilayered structures promising candidate for various alluring technological applications. This include the detection of magnetic bits in a hard drive disc (read head sensor of computer) and detection of magnetic field of a rotating object (anti-lock brake system in automobiles) and even applications in biomedical sensors. Different theoretical models were put forward to understand the origin of exchange coupling and their oscillatory behavior with spacer thickness. A clear explanation was given by quantum well model $(\mathrm{QW})$ and the RKKY model. The 
RKKY model suggests that the exchange coupling between two impurity spins is mediated by the spin polarized conduction electron of the non-magnetic spacer and the period of oscillation depends upon the thickness of spacer material and its crystallographic orientations. The amplitude ratio and phase of oscillation are also very well explained by RKKY perturbation theory taking the topology of Fermi surface and the degree of confinement within spacer layer in to account. Based upon RKKY theory and taking other important factors like surface roughness, thickness, geometry as well as the orientation of spacer layer into account, a transparent, but a qualitative explanation [7, 8] was given for the long periodic and multi-periodic oscillations of IEC observed in experiments. In addition, the thickness of magnetic layer is also found to have a bearing on the oscillations in IEC. The other QW model based on the quantum confinement within the ferromagnetic layers [72, 73, 74, 75] has also been successful in elucidating the observed oscillation of magnetic coupling. However, all these qualitative explanations could not satisfactorily elucidate the quantitative predictions like strength of the exchange coupling J, which significantly depends upon the degree of matching of energy bands at the interface. The ab-initio local spin density calculations based upon density functional theory [9, 10, 11, 12] was able to estimate the coupling strength with precision from total energy calculations. The calculated energy difference between FM and AFM configurations, which represents the inter layer coupling energy $J$ showed a slowly decaying oscillation with the spacer layer thickness. Furthermore, this was proved to have greater accuracy with experimental results [13, 14, 15] and was in good agreement with the RKKY model. All these seminal find- 
ings contributed to the understanding of IEC as well as GMR in bulk multilayer structures. However, for the next generation ultra high density memory devices and magnetic sensors in sub-micron dimensions, it is necessary to extrapolate the presently used thin film to a new class of spintronic devices in submicron dimension.

One dimensional multilayered nanowire structures have shown tremendous promise in this regards. They are constructed from bulk multilayered structure. The first question needs to be answered is: Do these miniaturized multilayered structures exhibit GMR effect? The answer is "Yes". Recently, one dimensional (1-D) magnetic/non-magnetic multilayered nanowires exhibiting GMR effect at ambient temperature[16, 17, 18] is demonstrated creating a breakthrough for the future magneto electronic device application. For example, using electro-deposition technique, Blondel et al. [16] have synthesized the $\mathrm{Co} / \mathrm{Cu}$ multilayered nanowire, and have observed the GMR effect up to $14 \%$ in the nanowire at room temperature. Other experimental groups like Katine et al. [19] have recently demonstrated the dependence of GMR effect on spacer width in $C o / C u$ system. Liu et al. [20] have reported the fabrication of $\mathrm{Co} / \mathrm{Cu}$ nanowires of different diameter. Chien et al [21] have shown the fabrication of Ni nanowires with the diameters in the range of $5 \mathrm{~nm}$ to $10 \mathrm{~nm}$ using highly uniform diamond shaped nanopore template for their potential application in biotechnology. Park has reported [22] the stress induced martenisitic phase transformation in $\mathrm{Ni} / \mathrm{Al}$ hybrid nanowire, and has shown this nanowire can outperform the bulk $\mathrm{Ni} / \mathrm{Al}$ as a shape memory alloy due to recoverable inelastic tensile stress property. The fabrication of $\mathrm{Co} / \mathrm{Cu}$ multilayered nanowire with $\sim 350$ repetitions of $\mathrm{Co} / \mathrm{Cu}$ sequences 
have been reported [23]. Using a different approach based on lithography, Urazhdin et al. [24] have reported the fabrication of $\mathrm{Ni}_{84} \mathrm{Fe}_{16} / \mathrm{Cu}(\mathrm{Pt}) / \mathrm{Ni}_{84} \mathrm{Fe}_{14}$ nanopillars and have demonstrated current driven switching effect in the nanopillars. The fabrication of single crystalline $\mathrm{Ni}-\mathrm{Cu}$ alloy nanowire by electro-depositing through the hexagonally ordered nanochanels has also been reported [25]. This $\mathrm{Ni} / \mathrm{Cu}$ alloy nanowire has potential applications in magnetic recording device. Various experimental groups have also reported the electronic and magnetic properties of $F e / P t$ nanoparticles and nanoalloy [26, 27, 28, 29]. The fabrication of $\mathrm{Fe} / \mathrm{Pt}$ multilayered nanowired structures [30, 31, 32] have also been reported. But the precise fabrication of multilayered nanowires with the desired magnetic and non-magnetic layer sequence is a serious challenge, which has been hindering their practical applications. The magnetic and magneto transport properties of the multilayered nanowires are highly dependent on their size, shape, crystallinity and spacer thickness. Thus, the controlled fabrication of these $1 D$ magnetic nano-materials are absolutely necessary. In a pioneering effort, Choi et al. [33] have recently reported the mass fabrication of free standing 1D Co/Pt multilayered or barcode nanowires with well defined layer thicknesses using programmable template-assisted deposition technique. In addition, using electric pulse deposition technique for varying the magnetic and non-magnetic layer thickness and applying magnetic field both along the axis and perpendicular to the axis of the wire, different values of GMR (4\% and 10\%) are reported [23]. Other experimental groups have also reported controlled fabrication of $\mathrm{Co} / \mathrm{Pt}$ multilayered nanowire [34], opening up a new vista for their potential application in magneto-electronics or spintronics. 


\subsection{Open questions}

Despite this experimental progress in fabrication [15, 16, 17, 18] and characterization of multilayered magnetic nanowire, only limited attention has been given to understand the most crucial atomic scale structural heterogeneity of the magnetic multilayered nanowires and its role in modulating the magnetic properties of the nanowire. Particularly, first principle theoretical calculations, which have been proved to have predictive capability, have not been reported to understand the role of the non-magnetic spacer in modulating the magnetic properties of $1 \mathrm{D}$ multilayered nanowire structures. The challenge associated with the first-principle calculations of isolated $1 D$ multilayered nanowire lies in the fact that one needs to consider a large supercell with sufficient vacuum to ensure negligible interaction between the nanowire and its image, and to allow several spacer layers to be embedded in the nanowire structures. This increases the computational time significantly considering the spin-polarized nature of the problem. Furthermore, no a priori information is available on the atomic structure or stability of these nanowires compounding a theoretical task of obtaining the equilibrium structures, and hence the magnetic properties. 


\subsection{My Contributions}

My aim has been to use ab initio theory to develop a basic understanding of the role of the nonmagnetic spacer layer in tuning the magnetic properties of these multilayered barcode nanowires. The research is focused on addressing many fundamental questions: Are these $1 D$ nanowires stable? Is is possible to tune the magnetic properties of the nanowires by varying the thickness of the nonmagnetic spacers? Is it possible to tune the IEC in this $1 D$ nanowire structure by controlling the spacer layer thickness? Does the IEC depend upon the nature of the spacer? Is the proposed model for the bulk multilayered systems (RKKY model)valid for nanoscale multilayered structures? To address these important questions, a variety of multilayered nanowire systems $(\mathrm{Fe} / \mathrm{Pt}, \mathrm{Fe} / \mathrm{Pd}, \mathrm{Fe} / \mathrm{Cu}, \mathrm{Fe} / \mathrm{Au}, \mathrm{FeAg})$ are considered in this project. I have used first-principles approach to look into subtle change in the magnetic and electronic properties of the wire with the change in the width of the nonmagnetic spacer. Particularly, I am addressing how the atomic level structural heterogeneity at the interface affects their magnetic property. I have performed periodic density functional calculation within the spin polarized local density functional approximation(LSDA) [35] to probe the compositional dependent magnetic and electronic properties of the nanowires. The Vienna ab initio simulation code (VASP) [38, 39] that uses plane wave basis function and ultra-soft pseudo potential to describe the valance core interaction, is utilized for our calculations. This thesis is organized as follows. The theoretical procedure used in this study is described in Chapter II. Chapter III discusses about the ferromagnetism in multi- 
layered nanowire system. Chapter IV will be focused on "Controlling IEC with thickness of the spacer width". Chapter V will compare IEC in different multilayered systems. In Chapter VI, I will conclude my project with a brief summary and suggestions for future work. 


\section{Chapter 2}

\section{Quantum many-body theory for solid}

\section{state systems}

\subsection{Introduction}

Solid state systems are quantum many-body systems comprised of large number of atoms and electrons. Structural packing of atoms in a solid and nature of the electronic structure dictate their electronic, magnetic, and optical properties. However, the exact determination of the electronic structure of a many-electron interacting system is practically impossible. Except for a system with one electron, the analytical solution does not exist. Computational cost increases significantly with the increase of the number of electrons. Thus, a new model 
with approximations is absolutely necessary to address this complex many-body problem. In the following Section, an overview of various approximations that are being used to solve this computationally intractable many-body problem is presented.

\subsection{Schrödinger Equation}

The Schrödinger equation [40] named after Erwin Schrödinger is the fundamental equation in physics. It describes the properties of any non-relativistic time independent quantum many-body system using wave function representation of the electron and is represented by:

$$
\hat{H} \psi\left(r_{1}, r_{2}, \ldots \ldots \ldots . . r_{N}\right)=E \psi\left(r_{1}, r_{2}, \ldots \ldots . r_{N}\right)
$$

$\hat{H}, \psi$ and $E$ in eqn. 2.1 are the Hamiltonian, many body wave function, and the energy eigen value of the system respectively. A many-body system consists of electrons as well as nuclei, which interacts with each other. Thus, the Hamiltonian of such a system with M number of nuclei and $\mathrm{N}$ number of electrons can be represented as:

$$
\hat{H}=-\frac{\hbar^{2}}{2 m_{e}} \sum_{i=1}^{N} \nabla_{i}^{2}-\sum_{i, I} \frac{Z_{I} e^{2}}{\left|r_{i}-R_{I}\right|}+\frac{1}{2} \sum_{i \neq j} \frac{e^{2}}{\left|r_{i}-r_{j}\right|}-\sum_{I=1}^{M} \frac{\hbar^{2}}{2 M_{I}} \nabla_{I}^{2}+\frac{1}{2} \sum_{I \neq J} \frac{Z_{I} Z_{J} e^{2}}{\left|R_{I}-R_{J}\right|}
$$

$\mathrm{M}, \mathrm{Z}$, and $\mathrm{R}$ are the mass, charge, and position vector of the nuclei; $m_{e}, e$ and $r$ represents mass, charge and position vector of electrons respectively. The first and fourth 
terms in eqn. 2.2 represent the kinetic energy contribution from electrons and nuclei. The second, third, and fifth terms are the potential energy terms resulting from ion-electron attraction, electron-electron repulsion, and ion-ion repulsion respectively. To predict the accurate electronic and geometric structure of a many-body system, one first needs to solve the Schrdinger equations and calculate the total energy of the system.Afterwords, the true ground state energy is obtained by minimizing energy with respect to the optimized nuclear and electronic co-ordinates. It is difficult to solve eqn. 2.2 without further approximations. The first simplification of eqn.2.2]is by Born and Oppenheimer [41] in 1927.

\subsection{Born-Oppenheimer Approximation}

This approximation stems from the fact that the mass of the nuclei are about $10^{3}$ times heavier than the mass of the electrons. Thus, classically, the electrons move much faster as compared to nuclei and the nuclei appear stationary to electrons. Hence, the electrons experience a fixed frozen-in potential due to the nuclei. This is sometimes referred to as

the adiabatic approximation. After adopting this adiabatic approximation, the eqn. 2.2 is reduced to an electronic Hamiltonian:

$$
\hat{H}=\sum_{i=1}^{N} \frac{\nabla_{i}^{2}}{2}+\sum_{i, I} \frac{Z_{i}}{\left|r_{i}-R_{I}\right|}+\frac{1}{2} \sum_{i \neq j} \frac{1}{\left|r_{i}-r_{j}\right|}
$$


Atomic units are used in eqn. 2.3. Even after this simplification, solving the eqn. 2.3. is still a complex problem because of the electron-electron interaction term. To decouple the many-body problem to a solvable one electron problem, the many-electron Hamiltonian in eqn. 2.3 needs to be reduced to an effective one electron Hamiltonian. This can be achieved either by using Hartree-Fock theory [43] or Density Functional Theory (DFT). Beyond these two theories, further approximations are necessary to deal with system having large number of electrons. For example, a periodic system, which is the main focus of this thesis, have infinite number of electrons. To deal with such a system I have used pseudo-potential approximation for electron-nuclei interaction, and the supercell approach to deal with the periodicity of the system. In the following Sections, I review various methods including the Hartree-Fock approach for completeness.

\subsection{Electron-electron interaction}

\subsubsection{Hartree-Fock Theory}

This approximation proceeds with the assumption that each electron appears to move in the average potential produced by other electrons in the presence of fixed nuclear charges. The total electronic wave function is approximated by product of single particle orthonormal orbitals taking anti-symmetry property of the wave function into account that leads to the 
exchange interaction. Slater [42] is able to represent the Hartree-Fock wave function in a more efficient $N \times N$ determinant form, known as Slater determinant, where orbitals are subjected to orthonormal constraints,

$$
\int \psi_{i}^{*}(r) \psi_{j}(r) d r=\left\langle\psi_{i} \mid \psi_{j}\right\rangle=\delta_{i j}
$$

The, Slater determinant form of many electron wave-function $\psi$ is expressed as:

$$
\psi=\frac{1}{\sqrt{N} !}\left|\begin{array}{ccccc}
\psi_{1}\left(r_{1}\right) & \psi_{2}\left(r_{1}\right) & \ldots & \ldots & \psi_{N}\left(r_{1}\right) \\
\psi_{1}\left(r_{2}\right) & \psi_{2}\left(r_{2}\right) & \ldots & \ldots & \psi_{N}\left(r_{2}\right) \\
\ldots & \ldots & \ldots & \ldots & \ldots \\
\ldots & \ldots & \ldots & \ldots & \ldots \\
\psi_{1}\left(r_{N}\right) & \psi_{2}\left(r_{N}\right) & \ldots & \ldots & \psi_{N}\left(r_{N}\right)
\end{array}\right|
$$

$\psi_{1}\left(r_{1}\right) \ldots \ldots \psi_{N}\left(r_{N}\right)$ represent the spin molecular orbitals; $\frac{1}{\sqrt{N !}}$ is the normalization constant; $\mathrm{N}$ represents the total number of electrons in the system. Now the many-electron Hamiltonian in eqn. 2.3 can be expressed as sum of one-electron Hamiltonian:

$$
H_{e}=\sum_{i} H_{H F}(i)
$$

$$
H_{H F}(i)=H_{0}(i)+V_{e}(i)
$$


Here $H_{0}(i)$ is the sum of electron kinetic energy and electron-ion interaction energies; $V_{i}(i)$ represents the average potential that the $i^{t h}$ electron experiences due to the interaction with the all other electrons in the system. The variational procedure is then adopted to determine the effective one electron Hamiltonian $H_{H F}(i)$ and hence the eigen value and eigen function.

\subsubsection{Correlation problem in Hartree-Fock approach}

Hartree-Fock theory $[43,44]$ has its own draw back since the single determinant wave function contains no correlation effect beyond the requirement by Pauli's exclusion principle. Only for a very weakly correlated system, the single determinant can represent the true wave function. But in a real system, the electrons are strongly correlated than that described by Hartree-Fock theory; the interaction energy missed by Hartree-Fock method is the correlation energy $E_{c}$, given by:

$$
E_{c}(i)=E_{0}(i)-E_{H F}
$$

$E_{0}$ is the exact ground state energy and is always higher than the non-relativistic energy i.e. $E_{H F}>E_{0}$. Thus, the correlation energy $E_{c}$ is a negative quantity. In order to take the correct correlation between the electrons into account, one needs to go beyond the HartreeFock theory. The alternate approaches are Configuration interaction theory(CI), many-body 
perturbation theory(MBPT), and the Density functional theory(DFT). CI and MBPT are only useful for atomic and simple molecular system with only few electrons. However, the DFT approach, which includes correlation among electrons, can be adopted efficiently for a larger system. Since DFT is used through out this thesis, a detailed discussion about this theoretical approach is given in the next Section.

\subsubsection{Density Functional Theory}

The DFT, in principle, is an exact theory of electronic structure. It is based on the electron density distribution $n(r)$, instead of the complicated many electron wave function

$\psi\left(r_{1}, r_{2}, \ldots \ldots \ldots r_{N}\right)$. Over the past few years, DFT [45, 46, 47] has become the physicist's method of choice for understanding the electronic structure of molecules, clusters and solids - any system consisting of nuclei and electrons. Here, I outlined the basic ideas of DFT. The history of DFT begins with the work of Thomas and Fermi [49] in 1920.

\subsubsection{Thomas-Fermi Model}

This model makes a significant conceptual presumption by taking the much simpler electron density $n(r)$ as the unknown variable rather than the $\mathrm{N}$-electron wave function. It reduces the $3 \mathrm{~N}$ degrees of freedom problem to a simple 3 degrees of freedom system. In this approximation, electrons are treated as independent particles and the electron-electron 
interaction energy is represented by the electrostatic (Columbic) energy $E_{e s}$ :

$$
E_{e s}[n(r)]=\frac{1}{2} \int d r \int d r^{\prime} \frac{n(r) n\left(r^{\prime}\right)}{\left|r-r^{\prime}\right|}
$$

The electronic kinetic energy associated with the system of non-interacting electro, $\mathrm{n} T[n(r)]$ :

$$
\begin{array}{r}
T[n(r)]=\int d r t[n(r)] \\
=C_{f} \int n(r)^{\frac{5}{3}} d r
\end{array}
$$

$t[n]$ is the kinetic energy functional for the system of non-interacting electrons within a homogeneous medium with density $n(r) ; C_{f}=\frac{3}{10}\left(3 \pi^{2}\right)^{\frac{2}{3}}$. The derivation is given in the App. 6.2. The classical electron-nucleus attraction energy is:

$$
\begin{aligned}
E_{\text {ext }} & =\int d r n(r) V_{\text {ext }} \\
& =-Z \int \frac{n(r)}{r} d r
\end{aligned}
$$

Thus the energy functional of the Thomas-Fermi N-electron atom in terms of electron density is given by:

$$
E_{T}[n(r)]=T[n(r)]+E_{e s}[n(r)]+E_{e x t}[n(r)]
$$


The energy functional $E_{T}[n(r)]$ is minimized under the constraint

$$
\int d r n(r)=N
$$

From the Lagrangian multiplier method:

$$
E_{T}[n(r)]+\mu N=T[n(r)]+E_{e s}[n(r)]+\int d r n(r)\left[V_{e x t}[n(r)]+\mu\right]
$$

The ground state electron density $t[n(r)]$ must satisfy the variational principle:

$$
\delta\left\{E_{T}[n(r)]-\mu\left(\int n(r) d r-N\right)\right\}=0
$$

This yields Euler- Lagrange equation:

$$
\mu=\frac{\delta E_{T}[n(r)]}{\delta[n(r)]}=\frac{\delta T[n(r)]}{\delta r}-\phi(r)
$$

$\phi(r)$, the electrostatic potential at a point due to the nucleus and the electrons, is given by:

$$
\phi(r)=\frac{z}{r}-\int \frac{n\left(r^{\prime}\right)}{\left|r-r^{\prime}\right|}
$$

This is the simple Thomas-Fermi model. Due to severe deficiencies associated with the rough description of the charge density and the electrostatic potential, Thomas-Fermi model 
is considered as a over simplified model; this does not have much importance for the quantitative prediction of solid state physics. The situation is improved by the landmark publication of Hohenberg and Kohn(1964). Two simple but powerful theorems [50] establish that the electron density $n(r)$ is the central quantity for describing electron interaction.

\subsubsection{Hohenberg-Kohn theorems}

The Hohenberg-Kohn theorems open new prospectives to the calculation of electronic properties of condensed system. For a system of $\mathrm{N}$ electrons, the ground state properties are entirely determined by the number of electrons and the position of the nuclei that gives the potential of the system [48]. The time independent Schrodinger equation for such a system can be expressed as:

$$
\hat{H} \psi\left(r_{i}\right)=E \psi\left(r_{i}\right)
$$

with

$$
\hat{H}=\hat{T}+\hat{V_{e e}}+\hat{V_{n e}}
$$




$$
\begin{array}{r}
\hat{T}=-\frac{1}{2} \sum_{i=1}^{N} \nabla_{i}^{2} \\
\hat{V_{e e}}=\sum_{i<j}^{N} \frac{1}{\left|r_{i}^{\prime}-r_{j}\right|} \\
\hat{V_{e x t}}=\hat{V_{n e}}=-\sum_{\alpha} \frac{Z}{\left|r-R_{\alpha}\right|}
\end{array}
$$

$V_{e e}$ is the electron-electron interaction potential and $V_{e x t}$ is the external potential. Hohenberg theorem uses the electron density $n(r)$ as the basic variable having one-to-one correspondence with the external potential $V_{\text {ext }}(r)$. Thus, the Hohenberg-Kohn theorem relates to any quantum mechanical system of $\mathrm{N}$ electrons moving under the influence of external potential $V_{\text {ext }}$, and can be stated as follows:

Theorem 1: The external potential $V_{\text {ext }}$ is uniquely determined by the electronic charge density $n(r)$. So the total energy is an unique functional of the density $n(r)$.

Thus the electron density $n(r)$ determines the many-electron, ground state wave function $\psi$ and hence the ground state electronic properties of the system. The total energy of the system is:

$$
E[n(r)]=T_{e}[n(r)]+V_{e e}[n(r)]+V_{e x t}[n(r)]
$$

$T_{e}$ and $V_{e e}$ are the kinetic energy of the electron and electron-electron interaction potential 
respectively. The external potential $V_{\text {ext }}$ takes the form:

$$
\begin{aligned}
V_{\text {ext }}[n(r)] & =\int V_{\text {ext }}(r) n(r) d r \\
V_{\text {ext }}(r) & =-\sum_{\alpha} \frac{Z_{\alpha}}{\left|r-R_{\alpha}\right|}
\end{aligned}
$$

Thus the energy functional takes the form as:

$$
E[n(r)]=\int V_{e x t}(r) n(r) d r+F_{H K}[n(r)]
$$

The functional $F_{H K}$ is:

$$
F_{H K}[n(r)]=T[n(r)]+V_{e e}[n(r)]
$$

with

$$
V_{e e}[n(r)]=V_{H}[n(r)]+E_{x c}(\text { non }- \text { classical term })
$$

Here $V_{H}[n(r)]$ is the classical electron-electron repulsion term also called Hartree potential. The non-classical part in eqn.2.24 is a very important quantity and is the exchange and correlation energy. The second Hohenberg and Kohn theorem provides the energy variational principle and is stated as:

Theorem 2: The density which minimizes the energy is the ground state density and the minimum energy is the ground state energy. 
For a trial density $\bar{n}(r)$ satisfying the conditions:

$$
\bar{n}(r) \geq 0
$$

and

$$
\begin{gathered}
\int \bar{n}(r) d r=N \\
E_{0} \leq E[\bar{n}(r)
\end{gathered}
$$

$\min E[n(r)]=E_{0}$

The variational principle requires that the ground state density must satisfy the stationary principle in the same way as in the Thomas-Fermi theory. Thus from eqn. 2.15

$$
\delta\left\{E[n(r)]-\mu\left[\int n(r) d r-N\right]\right\}=0
$$

giving the Euler-Lagrange equations as:

$$
\mu=\frac{\delta E[n(r)]}{\delta n(r)}=V_{e x t}[n(r)]+\frac{\delta F_{H K}[n(r)]}{\delta n(r)}
$$

$\mu$ is the chemical potential. The eqn. 2.26 can represents the exact equation for the ground state electron density provided we know the exact functional $F_{H K}$. Thus the knowledge of $F_{H K}$ is necessary to solve any quantum mechanical system. The eqn. 2.27 looks very at- 
tractive because the calculation of electron density $n(r)$ is much simpler than the N-electron wave function. However, the accurate implementation of this method is not easy. The main problem is to get an explicit form of the functional $F_{H K}$. The calculations with drastic approximations to these functionals results in the loss of accuracy to a great extent.

\subsubsection{The Levy Constrained search}

The Hohenberg-Kohm theorem suffers from two major drawbacks. First, it assumes no degeneracy for the ground state of the system. Second, it assumes the density to be $v$-representable. Thus, if $n(r)$ is the density corresponding to the antisymmetric wave function of the ground state Hamiltonian, the correct formulation of variational principle is:

$$
E_{0}=\min _{n(r)} E_{v}[n(r)]
$$

$v$ stands for the $v$-representability of the density $n(r)$. But, the specific conditions to make a density $v$-representable are not known as many reasonable densities have been shown to be non-v-representable. A generalization of Hohenberg-Kohn theorem was found by Levy

[51, 52] using an alternative weaker constraint i.e $N$-representable density which is known as Levy constrained search formulation. The $v$ and Nrepresentability of $n(r)$ is discussed in Appendix B. The starting point is to distinguish the ground state wave function $\psi_{0}$ from different $\psi_{n 0}$, which also integrates to give the ground state electron density $n_{0}(r)$. From 
the variational principle:

$$
\left\langle\psi_{n 0}|\hat{H}| \psi_{n 0}\right\rangle \gg\left\langle\psi_{0}|\hat{H}| \psi_{0}\right\rangle=E_{0}
$$

Taking into account that $V_{\text {ext }}[n(r)]$ is the function of $n(r)$ only,

$$
\begin{gathered}
\left\langle\psi_{n 0}\left|\hat{T}+\hat{V}_{e e}\right| \psi_{n 0}\right\rangle+\int V_{e x t}(r) n(r) d r \gg \\
\left\langle\psi_{0}\left|\hat{T}+\hat{V_{e e}}\right| \psi_{0}\right\rangle+\int V_{e x t}(r) n(r) d r \\
\Rightarrow\left\langle\psi_{n 0}\left|\hat{T}+\hat{V}_{e e}\right| \psi_{n 0}\right\rangle \gg\left\langle\psi_{0}\left|\hat{T}+\hat{V}_{e e}\right| \psi_{0}\right\rangle
\end{gathered}
$$

Thus $\psi_{0}$, is the wave function that minimizes the kinetic energy as well as the electronelectron interaction energy. It also should integrate to give the ground state electron density $n_{0}(r)$. Then the functional becomes:

$$
F_{H K}[n(r)]=\min _{\psi \rightarrow n(r)}\left\langle\psi\left|\hat{T}+\hat{V}_{e e}\right| \psi\right\rangle
$$

$F_{H K}[n(r)]$ looks for all $\psi$ that gives the density $n_{0}(r)$. This gives the expression for energy 
as:

$$
\begin{array}{r}
E_{0}=\min _{n(r)}\left[F_{H K}[n(r)]+\int V_{\text {ext }}(r) n(r) d r\right] \\
=\min _{n(r)} E[n(r)]
\end{array}
$$

$E[n(r)]$ searches over all $N$-representable densities. Thus the $v$-representable problem can be avoided and the explicit form of the energy is:

$$
\begin{array}{r}
E_{0}=\min _{n(r)}\left[\min _{\psi_{n 0} \rightarrow n(r)}\left\langle\psi_{n 0}\left|\hat{T}+\hat{V}_{e e}\right| \psi_{n 0}\right\rangle\right. \\
\left.+\int V_{e x t}(r) n(r) d r\right]
\end{array}
$$

The inner minimization restricts all the wave functions $\psi_{n 0}$ leading to the ground state density $n_{0}(r)$. The outer minimization searches over all densities that integrate to give total number of electrons $\mathrm{N}$.

\subsubsection{The Kohn-Sham Formulations}

Though, Hohenberg-Kohn theorem gives the possibility to calculate the electronic properties of a quantum mechanical system using the ground state density $n(r)$, it doesn't describe the way to find it. The Kohn-Sham formulation [53]provides the solution for it. The ground state energy of many-electron system is the minimum of the energy functional. So 
the equation takes the form as

$$
\left.E[n(r)]=F[n(r)]+\int V_{\text {ext }}(r) n(r) d r\right]
$$

The Kohn-Sham approach provides an explicit approximation for the functional $F[n(r)]=$ $T[n(r)]+V_{e e}[n(r)]$ by introducing orbitals concept into the problem in such a way that the kinetic energy can be computed with a greater accuracy.

Non-interacting $v$-representability: In this method, they introduce an auxiliary non-interacting reference system, which intends to imitate the actual many-electron system. The Hamiltonian of such an auxiliary system can be written as [46]:

$$
\hat{H_{k s}}=\sum_{i}^{N}\left(-\frac{1}{2} \nabla_{i}^{2}\right)+\sum_{i}^{N} V_{k s}(r)
$$

The electron-electron repulsion term is switched off, and the electrons move in presence of an auxiliary external potential $V_{k s}(r)$ known as Kohn-Sham potential. The potentials are defined in such a way that the ground state electron density $n(r)$ is exactly equals to the density $n_{0}(r)$ of the electronic system for which the functional $F[n(r)]$ needs to be evaluated. Since there is no interaction between the particles, the ground state wave function is less complicated compared to the wave function of an actual interacting system. Thus the ground state wave function can take an explicit form of a single particle wave function satisfying the anti-symmetry condition. The single particle wave function is represented by 
a Slater determinant as:

$$
\psi_{k s}=\frac{1}{\sqrt{N !}} \operatorname{det}\left[\begin{array}{lll}
\psi_{1} & \psi_{2} \ldots \ldots \ldots \psi_{N}
\end{array}\right]
$$

$\psi s$ are the $\mathrm{N}$ lowest eigenstates of the one-electron Hamiltonian $h_{s}$

$$
h_{s} \psi_{i}==\left[-\frac{1}{2} \nabla^{2}+V_{k s}(r)\right] \psi_{i}=\varepsilon_{i} \psi_{i}
$$

These single particle wave functions $\psi_{i}(r)$ are called Kohn-Sham orbitals. For each spinup orbital, there will be a spin-down orbital with same eigen function and eigen value. Because of the degeneracy, we deal with Kohn-Sham special orbitals $\psi_{k s}$. For the Nparticle quantum mechanical system, we have either $\frac{N}{2}$ Kohn-Sham orbitals for even $\mathrm{N}$ or $\frac{N}{2}+1$ for odd N. For odd $\mathrm{N}$, the highest orbital is the unpaired orbital. The ground state kinetic energy of this auxiliary system for the occupied orbitals is expressed as [46, 55]:

$$
T_{k s}[n(r)]=\sum_{i=1}^{N}\left\langle\psi_{k s}\left|-\frac{1}{2} \nabla_{i}^{2}\right| \psi_{k s}\right\rangle
$$

The kinetic energy $T_{k s}[n(r)]$ is still not the exact ground state kinetic energy functional $T[n(r)]$. The normalized N-representable density is decomposed as:

$$
n(r)=\sum_{i}^{N} \sum_{s}\left|\psi_{k s i}(r, s)\right|^{2}
$$


For this auxiliary system, the functional $F_{k s}$ takes the expectation value of the non-interacting kinetic energy $T_{k s}[n(r)]$ and the non-interacting internal potential energy $V_{k s}(r)$.

$$
F_{k s}=T_{k s}[n(r)]+V_{k s}(r)
$$

The potential energy $V_{k s}(r)$ is sum of the of Hatred energy $V_{H}$ and the exchange energy $V_{X}$,

$$
V_{k s}=V_{H}+V_{X}(r)
$$

The $V_{H}$, electron-electron Coulombic energy takes the form

$$
V_{H}[n(r)]=\frac{1}{2} \int d r \int d r^{\prime} \frac{n(r) n\left(r^{\prime}\right)}{\left|r-r^{\prime}\right|}
$$

$V_{X}$, the exchange energy is due to the quantization of charge and the anti-symmetry property of the electronic wave function with respect to the exchange of co-ordinates between any two particles. This is expressed as:

$$
V_{X}[n(r)]=-\frac{1}{2} \sum_{i, j} \int d r \int d r^{\prime} \frac{\psi_{k s i}^{*}(r) \psi_{k s i}\left(r^{\prime}\right) \psi_{k s j}^{*}\left(r^{\prime}\right) \psi_{k s j}(r)}{\left|r-r^{\prime}\right|}
$$

The difference between the actual energy functional $F[n(r)]$ and the auxiliary functional $F_{k s}[n(r)]$ is called the correlation energy $E_{c}$.

$$
E_{c}=F[n(r)]-F_{k s}[n(r)]
$$




$$
E_{c}=T[n(r)]-T_{k s}[n(r)]+V_{e e}[n(r)]-V_{H}-V_{X}(r)
$$

To understand the correlation energy [46, 55], let us imagine a the non-interacting auxiliary system and then switch on the interaction energy, which is mostly the electron-electron repulsion energy. The system considered is in the ground state with fixed electron density. Now due to mutual repulsion, the electrons will avoid each other more at their close proximity than that they would in the non-interacting system. As a result, the internal potential energy is reduced since electron pairs are less likely to be closer to each other. For the greater mutual repulsion, the wave function gets changed resulting in an increase in the kinetic energy. Thus, the correlation energy is explained by both decrease in potential and increase in kinetic energy component. and the eqn. 2.46 is written as:

$$
E_{c}=T_{c}+V_{c}
$$

$T_{c}$ is the difference between the exact kinetic energy and Kohn-Sham Kinetic energy, and assumed to be fairly small. $V_{c}$ is the potential part of the correlation energy. The calculation of exchange energy, $V_{X}$ eqn.2.44 is computationally expensive compared to the Hatred energy, and the non-interacting kinetic energy. Thus the exchange and the correlation energies are always approximated by combining them together as the exchange-correlation energy, $E_{x c}$.

$$
E_{x c}=V_{X}+E_{c}
$$


Now rearranging them, we get:

$$
\begin{array}{r}
E_{x c}=V_{X}+F-F_{k s} \\
=V_{X}+F-\left[T_{k s}+V_{H}+V_{X}\right] \\
=F-T_{k s}+V_{H}
\end{array}
$$

Thus in the Kohn-Sham frame of reference, the exact form of the functional $F[n(r)]$ is:

$$
F[n(r)]=T_{k s}[n(r)]+V_{H}[n(r)]+E_{x c}[n(r)]
$$

with the exact expression for exchange-correlation energy as:

$$
E_{x c}[n(r)]=T[n(r)]-T_{k s}[n(r)]+V_{e e}[n(r)]-V_{H}[n(r)]
$$

Now the energy functional for the $\mathrm{N}$-particle system is:

$$
E[n(r)]=T_{k s}[n(r)]+V_{H}[n(r)]+E_{x c}[n(r)]+\int n(r) V_{e x t}(r) d r
$$

From Euler's eqn. 2.27

$$
\mu=\frac{\delta T_{k s}[n(r)]}{\delta n(r)}+V_{e f f}[n(r)]
$$


$V_{\text {eff }}$ is the Kohn-sham effective potential

$$
\begin{aligned}
V_{e f f}[n(r)] & =V_{e x t}(r)+\frac{\delta V_{H}[n(r)]}{\delta n(r)}+\frac{\delta E_{x c}[n(r)]}{\delta n(r)} \\
& =V_{e x t}(r)+\int \frac{n(\dot{r})}{\left|r-r^{\prime}\right|} d r^{\prime}+V_{x c}[n(r)]
\end{aligned}
$$

with the exchange-correlation potential,

$$
V_{x c}[n(r)]=\frac{\delta E_{x c}[n(r)]}{\delta n(r)}
$$

Thus, eqn. 2.52 represents the energy in terms of $\mathrm{N}$ orbitals which are allowed to vary over the entire space so that the density of eqn. 2.40 covers all $\mathrm{N}$-representable densities. To have a finite kinetic energy, the orbitals are constrained to be orthonormal

$$
\int \psi_{i}^{*}(x) \psi_{j}(x) d x=\delta_{i j}
$$

A functional $\Omega$ is defined as:

$$
\Omega\left[\psi_{i}\right]=E[n(r)]-\sum_{i}^{N} \sum_{j}^{N} \varepsilon_{i j} \int \psi_{i}^{*}(x) \psi_{j}(x) d x
$$


$\varepsilon_{i j}^{\prime} s$ are the Lagrange multipliers and $E[n]$ is the energy functional of $\psi_{i}$ and can be expressed as:

$$
\begin{array}{r}
E[n]=\sum_{i}^{N} \sum_{s} \int \psi_{i}^{*}(r)\left(-\frac{1}{2} \nabla^{2}\right) \psi_{i}(r) d r \\
+V_{H}[n]+E_{x c}[n]+\int n(r) V_{\text {ext }}(r) d r
\end{array}
$$

For $E[n]$ to be minimum, the required condition is:

$$
\Omega\left[\psi_{i}\right]=0
$$

This gives us the Kohn-Sham equation:

$$
h_{e f f} \psi_{i}==\left[-\frac{1}{2} \nabla^{2}+V_{e f f}(r)\right] \psi_{i}=\sum_{j}^{N} \varepsilon_{i j} \psi_{j}
$$

Here the effective one-electron Hamiltonian $h_{\text {eff }}$ is a Hermitian operator and $\varepsilon_{i j}$ is a Hermitian matrix. $\varepsilon_{i j}$ can be diagonalized by unitary transformation of orbitals. This keeps the ground state single determinant wave function (eqn. 2.37), the ground state density eqn. 2.40 and the Hamiltonian invariant under such transformations. Thus the canonical from of the Kohn-Sham [46] orbital equation is reduced to its simplest form as:

$$
\left[-\frac{1}{2} \nabla^{2}+V_{e f f}(r)\right] \psi_{i}=\varepsilon_{i} \psi_{i}
$$


$V_{\text {eff }}$ given by eqn. 2.54, depends on $n(r)$, given by eqn. 2.40, Thus the Kohn-Sham Schrödinger eqn.2.61 the effective potential $V_{\text {eff }}$ and $n(r)$ must be solved self-consistently. In self-consistent approach, we start with a suitable $\psi$ to obtain $n(r)$; construct $V_{e f f}$; solve eqn. 2.61to obtain a new set of $\psi_{s}$ to get new $n(r)$; mix the old and the new $n(r)$; continue the procedure till a convergence in $n(r)$ is achieved. The total energy is then determined from the final density $n(r)$,

$$
E=\sum_{i}^{N} \varepsilon_{i}-V_{H}[n]-E_{x c}[n]-\int n(r) V_{e x t}(r) d r
$$

Thus, by introducing N-orbitals it is easier to handle the kinetic energy part $T_{k s}[n(r)]$, which is the dominant part of the exact kinetic energy $T[n(r)]$. Though all Hartree, HartreeFock and Kohn-Sham theories provide effective one-electron equations to describe a manyelectron system, the Kohn-Sham method is exact in principle and distinguished from the other two theories as it incorporates fully the exchange-correlation effects of electrons. Kohn-Sham equation would give the exact solution for $n(r)$ and $E[n(r)]$ provided the exchange-correlation energy $E_{x c}$ is precisely known.

\subsubsection{Approximation for the exchange-correlational functional}

One of the major problems in DFT is that the exact form of the exchange and correlation functionals are not known except for the free electron gas. To get a better picture of the approximation to these functionals, we can write the total energy of the electronic system 
[54, 55] in terms of different energy contributions.

$$
E[n]=T_{k s}+E_{\text {ext }}+E_{H}+E_{X}+E_{c}
$$

The interaction of electrons with the external field $E_{\text {ext }}$, the classical electron-electron interaction energy $E_{H}$, are explicit function of the ground state density $n(r)$. The non-interacting kinetic energy $T_{k s}$, and the exchange energy $E_{X}$ are the functional of the non-interacting orbitals; thus unknown functionals of $n(r)$. The correlation energy, $E_{c}$, is unknown. No exact approximation is known to achieve a accuracy level for $E_{c}$ comparable to the other terms. Even if the exchange energy is treated exactly as functional of orbitals, as done in Hartree-Fock theory, the errors introduced while approximating correlation energy can't be compensated. Thus it is a good idea to treat exchange and correlation energy at a similar level of approximation, and combine them as exchange-correlation energy $E_{X C}$. For the homogeneous electron gas, which is the simplest system representing correlated electrons, the exchange energy is given by the Dirac expression

$$
\begin{gathered}
\varepsilon_{x}[n]=-\frac{3}{4}\left(\frac{3}{\pi}\right)^{\frac{1}{3}} n^{\frac{1}{3}}=-\frac{0.458}{r_{s}} \\
r_{s}=\left(\frac{3}{4 \pi n}\right)^{\frac{1}{3}}
\end{gathered}
$$

represents the mean inter-electronic distance. The best approximation for correlation energy is obtained from the Monte-Carlo simulations of both spin-polarized and spin-unpolarized 
homogeneous electron gas, and is expressed as:

$$
\begin{aligned}
\varepsilon_{x}[n]=A \ln r_{s}+B+C r_{s} \ln r_{s}+D r_{s}, & r_{s} \leq 1, \\
=\frac{\gamma}{\left(1+\beta_{1} \sqrt{r_{s}}+\beta_{2} r_{s}\right)}, & r_{s}>1
\end{aligned}
$$

For a molecular system, where the electron density is far different from ideal homogeneous electron gas, the most widely used approximation for $E_{X C}$ are Local density approximation(LDA) and the Generalized gradient approximation(GGA).

Local-density approximation: This is the simplest approximation method, implemented in Kohn-Sham formulation of DFT for describing the exchange-correlation energy of a many-electron system. The LDA assumes that $E_{X C}$ energy is purely a local functional, and applicable to a system where the charge density varies slowly. It assumes that the exchange-correlation energy per electron at point $r$ in the electron gas, $\varepsilon_{X C}$, is equal to exchange-correlation energy per electron in a homogeneous electron gas that has the same density as the true system of electron gas at point $r$. Thus,

$$
E_{X C}[n]=\int \varepsilon_{X C}(n) n(r) d^{3} r
$$

The Local spin density approximation(LSDA) is just the generalization of LDA, taking electron spin into account, and can be expressed as:

$$
E_{X C}[n \uparrow, n \downarrow]=\int \varepsilon_{X C}(n \uparrow, n \downarrow) n(r) d^{3} r
$$


Generalized gradient approximation: The GGA approximation is still a local functional but it takes the gradient of density at the same coordinate and expressed as:

$$
E_{X C}[n \uparrow, n \downarrow]=\int \varepsilon_{X C}(n \uparrow, n \downarrow, \nabla n \uparrow, \nabla n \downarrow) n(r) d^{3} r
$$

Usually, GGA approach gives very good result for optimized geometry and ground state energy of a many-body system. But for solid state calculation with Kohn-Sham theory, the local density approximations are commonly used along with the plane wave basis set.

\subsection{Supercell approach}

Up to now, we have been demonstrating that the Hartree-Fock and Density-Functional methods can be implemented to map a many-body problem into an effective one-particle problem. However for a bulk system, within these formalism we need to solve the time independent Schrödinger equations for infinite number of non-interacting electrons, that experience static potential due to infinite number of nuclei. This requires the calculation of wave function for each of the infinite number of electrons. Since each electronic wave function extends over the entire solid, we need an infinite basis set to represent the wave function. But this problem can be simplified if the infinite system is periodic. The periodic system allows us to simplify the problem by considering a simpler finite system called a supercell. The chosen supercell is large enough to contain all the information about the 
system with in it and gets replicated periodically throughout the entire space. The approach can be dealt using the Bloch's theorem [37, 56].

\subsubsection{Bloch's theorem}

For a perfect crystal at $0^{0} \mathrm{~K}$ the nuclei are arranged in a regular periodic manner and the system is invariant under translation by the Bravais lattice vector $\mathbf{R}$. The Bloch's theorem states that for a system of noninteracting particles moving in a static potential $\mathbf{V}$, which may be the Kohn-Sham potential $V_{k s}$, the potential $\mathbf{V}$ is invariant under the translation vector $\mathbf{R}$.

$$
V(\mathbf{r}+\mathbf{R})=V(\mathbf{r})
$$

Bloch's theorem uses this periodicity to reduce the infinite number of one-electron wave functions to a finite number that depends upon the number of electrons in the supercell. The electronic wave function is represented by the product of lattice periodic part $f_{j}(r)$ and a wave like part $e^{(i \mathbf{k} . \mathbf{r})}$. The function $f(\mathbf{r})$ has the same periodicity as that of lattice i.e. $f(\mathbf{r}+\mathbf{R})=f(\mathbf{r})$.

$$
\psi_{j, k}(\mathbf{r})=f_{j}(r) e^{i k . \mathbf{r}}
$$

Here $j$ is the band index and $\mathbf{k}$ is the wave vector in the first Brillouin zone of the reciprocal lattice. The lattice periodic part $f_{j}(\mathbf{r})$ is represented by a discrete plane waves basis set with 
the reciprocal lattice vector $\mathbf{G}$ of the crystal as the wave vector.

$$
f_{j}(\mathbf{r})=\sum_{\mathbf{G}} C_{j, \mathbf{G}} e^{(i \mathbf{G} . \mathbf{r})}
$$

with $\mathbf{G . R}=2 \pi m$ where $\mathbf{R}$ represents the crystal lattice vector; $\mathrm{m}$ represents an integer. Thus the electronic wave function is represented as:

$$
\psi_{j, k}(\mathbf{r})=\sum_{\mathbf{G}} C_{j, \mathbf{k}+\mathbf{G}} e^{[i(\mathbf{k}+\mathbf{G}) \cdot \mathbf{r}]}
$$

This electronic wave function is independent of the type of crystal. Due to periodicity of the crystal, instead of solving the wave function over the infinite space, one requires solution of the wavefunction only within the supercell. In the reciprocal space within the

first Brillouin zone of the supercell, there are infinite number of possible $k$ points at which the wave functions need to be calculated, as the occupied states at each $k$ point contributes to the electronic potential of the system. Thus, infinite number of calculations are needed to compute the potential. This problem can be simplified by sampling the entire Brillouin zone to a special set of k-points by imposing boundary conditions on the wave-functions.

\subsubsection{Brillouin zone sampling}

The infinite periodic system can be modeled by a large number of primitive cells $\left(N_{c}\right)$ stacked together. $N_{c}=N_{1} N_{2} N_{3}$, where $N_{i}$ cells are along $a_{i}$ direction; $i=1,2,3$. The 
periodic boundary condition to the wave functions can be understood as the particle leaves one surface of the crystal at the same instant it enters the crystal at the opposite surface. Thus

$$
\psi\left(\mathbf{r}+N_{i} \mathbf{a}_{i}\right)=\psi(\mathbf{r})
$$

with $i=1,2,3$. and using Bloch's theorem

$$
\psi\left(\mathbf{r}+N_{i} \mathbf{a}_{i}\right)=\psi(\mathbf{r}) e^{\left(i N_{i} \mathbf{k} \cdot \mathbf{a}_{i}\right)}
$$

From eqn.2.73 and eqn. 2.74

$$
\begin{array}{r}
\psi(r) e^{\left(i N_{i} k \cdot a_{i}\right)}=1 \\
e^{\left(2 \pi i N_{i} x_{i}\right)}=1, \quad i=1,2,3 .
\end{array}
$$

Then the values $x i=\frac{l i}{N i}$ with $l i$ are integers; the wave vector $\mathbf{k}=x 1 g 1+x 2 g 2+x 3 g 3=$ $\sum_{i} x_{i} g_{i}$ Thus the allowed $k$ values are:

$$
\mathbf{k}=\sum_{i=1}^{3} \frac{l_{i}}{N_{i}} g_{i}
$$

Though for a infinite prefect crystal the number of allowed $k$-vectors are still infinite, the k-vectors related by the reciprocal lattice vectors $\mathbf{G}$ are all equivalent and is expressed as: 
$\mathbf{k}^{\prime}=\mathbf{k}+\mathbf{G}$. Thus the wave-functions are restricted to those $k$-points lying within the first Brillouin zone. Since the occupied state at each $k$-point within the first Brillouin zone contributes to the potential of the solid, the eigen states of the Hamiltonian must be calculated for the infinite number of $k$-points in the first Brilloiun zone. However it is possible to take the advantage of the fact that the electronic wave function and the eigen values vary smoothly over a particular region of the Brillouin zone. Hence the wave function over a region of k-space can be represented by a wave function at single $k$-point. Thus finite number of $k$-points in the first Brillouin zone are needed to calculate the potential as well as total energy of the system. Different methods are devised by Chadi and Cohen [57], Monkhorst and Pack [58] to generate a sufficiently dense set of special $k$-points in the irreducible part of the Brillouin zone to reduce the error in calculating total energy of the system. The volume of the Brillouin zone $\Omega_{B Z}$ can be related to the volume of the supercell $\Omega_{S C}$ by the relation:

$$
\Omega_{B Z}=\frac{(2 \pi)^{3}}{\Omega_{S C}}
$$

For a large supercell, the volume of Brillouin zone is smaller and only few $k$-points are required to describe the variation of the wavefunction in the Brillouin zone. So the center of the Brillouin zone, at $k=0$, represented by $\Gamma-$ point is enough to calculate the wave function and the eigen values. Now the problem of infinite set of $k$-points is solved but the electronic wave function at each $k$-point needs to be expressed in terms of discrete basis set(plane waves) which is infinite. In the following section this problem is addressed. 


\subsubsection{Plane-wave basis sets}

In principle, for an exact energy calculation, the dimension of the plane wave basis set needs to be infinite. This infinite basis set can be truncated to a finite basis set by introducing particular energy cut-off. The kinetic energy of the plane waves, given by eqn. 2.72 is:

$$
\varepsilon=\frac{\hbar^{2}}{2 m}|\mathbf{k}+\mathbf{G}|^{2}
$$

The plane waves having lower $\varepsilon$ are more important than those with higher kinetic energy. Thus, for practical purposes the basis set includes only those plane waves that have kinetic energy less than a particular cutoff energy, $\varepsilon_{c u t}$. Thus, the application of Bloch's theorem makes the continuum plane wave basis set to be expanded in terms of discrete set of plane waves, which can be further reduced to a finite basis set by introducing the $\varepsilon_{c u t}$. The error in calculation of total energy with the truncated finite basis set can always be reduced by increasing the value of the cutoff energy.

\subsubsection{Plane-wave picture of Kohn-Sham equations}

The Kohn-sham equation in DFT takes a much simpler form in a periodic system where the electronic wave function is expanded in terms of plane wave basis set. The Fourier-space 
representation of the Kohn-Sham equation is [46]:

$$
H_{\mathbf{k}+\mathbf{G}, \mathbf{k}+\mathbf{G}}, C_{i, \mathbf{k}+\mathbf{G}},=\varepsilon_{i} C_{i, \mathbf{k}+\mathbf{G}^{\prime}}
$$

where

$$
\begin{array}{r}
H_{\mathbf{k}+\mathbf{G}, \mathbf{k}+\mathbf{G}},=\frac{1}{2}|\mathbf{k}+\mathbf{G}|^{2} \delta_{\mathbf{G}, \mathbf{G}^{\prime}} \\
+V_{\text {ext }}\left(\mathbf{G}-\mathbf{G}^{\prime}\right)+V_{H}\left(\mathbf{G}-\mathbf{G}^{\prime}\right)+v_{X c} V_{H}\left(\mathbf{G}-\mathbf{G}^{\prime}\right)
\end{array}
$$

Here the kinetic energy is diagonal; $V_{e x t}, V_{H}, V_{x c}$ are the Fourier component of external, Hartree, and exchange-correlation potentials respectively. The solution to the above KohnSham equation can be done by diagonalizing the Hamiltonian matrix $H_{\mathbf{k}+\mathbf{G}, \mathbf{k}+\mathbf{G}^{\prime}}$. The size of the matrix is determined by the choice of cutoff energy $\varepsilon_{c u t}$. However, for a large system with both valence and core electron the size of the required matrix is intractably huge irrespective of our choice of cutoff energy. Thus, a very large number of plane wave basis set is required to expand the tightly bound core orbitals as well as the rapid oscillation of valence electron wave functions in the core region. This problem can be handled by the pseudopotential approximation of the actual potentials. 


\subsection{Pseudopotential Approximation}

This approximation $[59,60,61]$ is based upon the observation that most physical properties of solids have a greater dependence on valence electron than the core electrons. Though the core electron has a larger contribution to the binding energy, it doesn't change when isolated atoms are brought together to form a crystal or molecule. The change in energy is mostly due to change in valence electron energies. Thus, if binding energy of core electron is excluded, the change in valence electron energies has a larger fraction of contribution to the total binding energy. The eigen states of an atomic Hamiltonian should be mutually orthogonal to each other. The core states are localized in the vicinity of the nucleus. The valence electron wave function oscillates rapidly in the core region to maintain orthogonality with the core states. Due to this rapid oscillation, the valence electrons have large kinetic energy in the core region that cancels the large potential energy due to strong nuclear potential. Computationally it is difficult to handle the strong nuclear Coulomb potential and the localized core electron wave functions. The strong oscillation of valence electron wave function in the core region also poses a serious challenge from the computational point of view. Thus, the idea of pseudopotential approach is to replace the strong Coulomb potential of the nucleus, and the effect of the tightly bound core electrons on valence electron by a much weaker effective pseudopotential shown in fig. 2.1 In addition, the valence electron wave functions that oscillate rapidly in the core region is represented by pseudo wave functions, that varies smoothly in the core region. The nuclear Coulomb potential, effective 
Pseudo potential $\left(V_{\text {pseudo }}\right)$, valence wave function and pseudo-wave function $\left(\psi_{\text {pseudo }}\right)$ are presented schematically in fig. 2.1

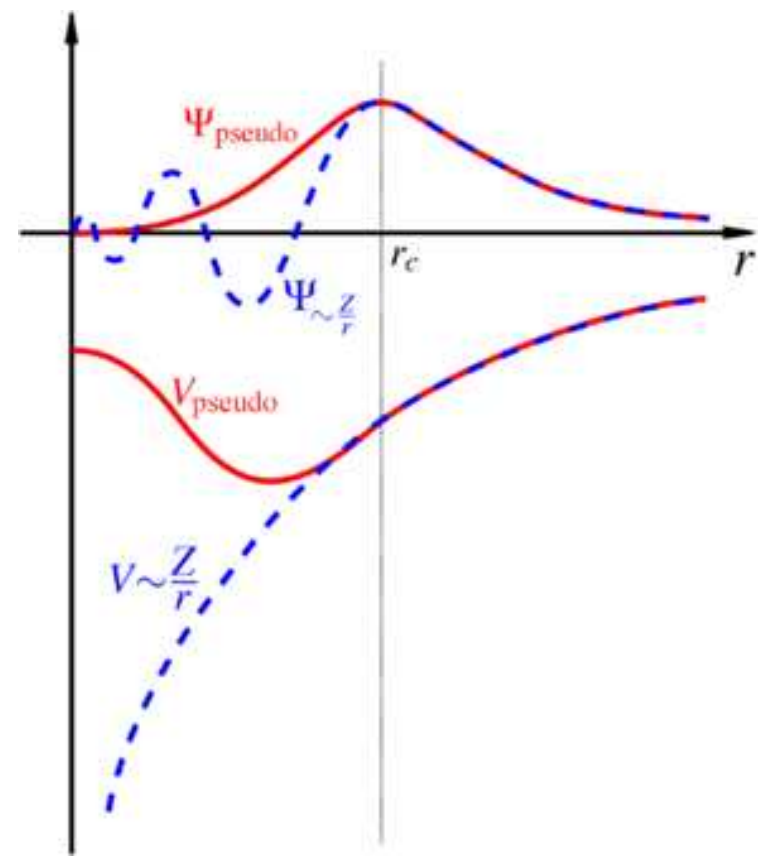

Figure 2.1: A schematic view of true ionic potential and valance wave function with the corresponding pseudopotential and pseudo-wave function(accessed on January 10, 2009; Copyright - Wquester, Wikipedia, 2006, distributed under public domain, refer Appendix $\mathrm{C}$ for permission.)

The pseudopotential is generated with certain criteria as follows. The scattering properties for the pseudo wave function, $\psi_{\text {pseudo }}$, are identical to that of the atomic wave function, $\psi$, from ionic core. $\psi_{\text {pseudo }}$ should be non-oscillatory in the core region defined by a cutoff radius $r_{c}$. $\psi_{\text {pseudo }}$ as well as it first and second derivative should be continuous at $r_{c}$, and outside the core region the pseudopotential, $V_{\text {pseudo }}$, should be identical to the actual ionic potential V; the scattering from the two potentials are indistinguishable. One of the major advantages of this method stems from the fact that $\psi_{\text {pseudo }}$ is free from radial nodes. Thus 
only a finite numbers of plane waves are sufficient to represent $\psi_{\text {pseudo }}$. Besides even if the total energy of the pseudo system is much more than the total energy of the true all electron system, the energy difference between the ionic configuration( meaningful for convergence test) in pseudo valence-electron system is as large that in all-electron system. This is true because the difference between the energies of different ionic configurations appears mostly in differences in energies of valence electrons. So, the accuracy required in calculating the energy difference between the ionic configuration in a pseudopotential approach is much smaller than that in all-electron approach.

\subsection{Computational Procedure for calculation of ground state charge density and energy}

First, the pseudopotential for the ions and the cutoff for the plane wave basis set needs to be chosen. Subsequently, $V_{H}$ and $V_{x c}$ are calculated by taking a guess charge density $n_{t r}(r)$, and at each required k-point the Hamiltonian matrix, $H$, is constructed and diagonalized to obtain the Kohn-Sham eigen states. From the Kohn-Sham eigen states, a new charge density $n_{n e w}$ is generated. The $n_{n e w}$ is mixed with $n_{t r}$ to obtain $n_{t r}$ for the next step. This procedure is repeated untill the self-consistency in the charge density is achieved. Once the final solution for $n$ is obtained, the total ground state energy is obtained with ground state density. So also the new Hamiltonian can be elucidated with freshly calculated potentials. 


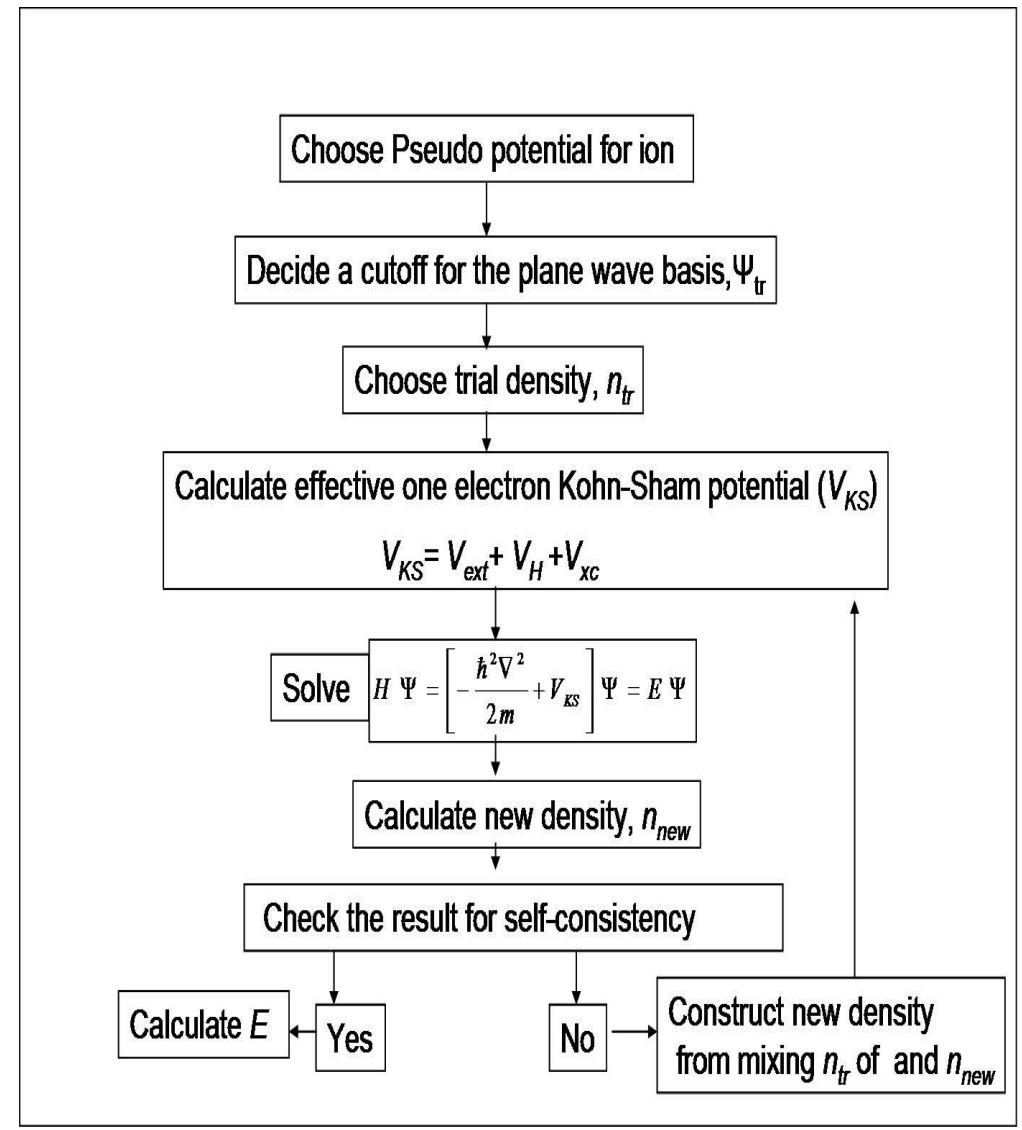

Figure 2.2: Flow chart for the computational approach 


\section{Chapter 3}

\section{Tuning ferromagnetism in multilayered}

\section{one dimensional barcode nanowires}

\subsection{Introduction}

The research on electronic and magnetic properties of hetero-structures has reached at a level from which the unifying theme of all seminal works, discussed in Chapter I, have contributed to the vast applications of magnetic and non-magnetic heterostructures in memory devices. The successful endeavor of several experimental groups in recent years demonstrating the phenomenal GMR effect in multilayered nanowire structures [16, 17, 18, 19,

20] and the precise fabrication of multilayered nanowire with a desired magnetic and non- 
magnetic layer sequence [33] have prompted intense research efforts towards using these one dimensional nano materials in nanoelectronics. Among all the multilayered nanowire systems, the $\mathrm{Fe} / \mathrm{Pt}$ system offers an exceptional promise for the future magnetoelectronic devices. This system has generated lot of research interest because of its multifaceted physical properties. Even in the absence of external magnetic field, the $\mathrm{Fe} / \mathrm{Pt}$ system can act as a permanent magnet. The other important physical properties like invar effect as well as the magnetostriction effect for this system are worth a mention. In addition, the successful fabrication of $\mathrm{Fe} / \mathrm{Pt}$ nanowire [30, 31, 32] gives more impetus towards studying the $\mathrm{Fe} / \mathrm{Pt}$ system. Various experimental groups have reported the electronic and magnetic properties of $\mathrm{Fe} / \mathrm{Pt}$ system in nanoparticles and nanoalloy [26, 27, 28, 29] forms. Though $a b$ initio calculations are performed to study electronic and magnetic properties in ferromagnetic (FM) and antiferromagnetic (AFM) configuration in the case of $F e / P t$ bulk alloy [62, 63], no first principle theoretical calculation has been used to understand the role of non-magnetic spacer in modulating ferromagnetic properties of one dimensional $\mathrm{Fe} / \mathrm{Pt}$ multilayer nanowire system. In the present study, I look into the role of non-magnetic Platinum spacer in tuning the ferromagnetism of $\mathrm{Fe} / \mathrm{Pt}$ nanowire at the ab initio level. I have also compared my results with other multilayered nanowire systems [64] like $\mathrm{Ni} / \mathrm{Al}$ and $\mathrm{Ni} / \mathrm{Cu}$. The rest of the Chapter is organized as follows: Sec. 3.2 summarizes the computational procedures in details; Sec 3.3 presents the results and discussions, and Sec. 3.3 contains a brief summary. 


\subsection{Computational approach}

The first principles calculation of isolated one dimensional multilayered nanowire structure is a difficult task because a large super cell with enough vacuum needs to be considered to guarantee negligible interaction between the nanowire and its image. Second, the spin polarized nature of the problem increases the computational cost significantly. Furthermore, it has always been a theoretical challenge to obtain the equilibrium structure of these multilayered nanowires since the atomic level structural details of these systems are not available beforehand. In this project, I have used bulk $f c c$ iron as the guiding point to construct the nanowire. Iron in $f c c$ phase is paramagnetic, and shows transitions to ferromagnetic phase with lattice expansion. I have chosen the $f c c$ phase because nanowires constructed from fcc phase have more number of nearest neighbor atoms compared to that of nanowires constructed from $b c c$ phase. Besides, successful controlled fabrication of Fe/Pt multilayer nanowire [30] in $f c c$ phase has also been reported. In addition, some other experimental groups [65] have shown that thin film of iron grows in fcc phase at $\mathrm{Fe} / \mathrm{Pt}$ interface. Again, electron diffraction studies have reported the $f c c$ structure for the $F e_{50} / P t_{50}$ nanoparticle

[66]. Taking all these factors into account, pristine iron nanowire structure was guessed from the $f c c F e$ in the (111) direction by repeating three conjugate planes (ABCABCABC) as shown in Figure. 3.1 a). Figure. 3.1 b) shows the [top view] arrangements of atoms of the first $\mathrm{ABC}$ series.

To construct our unit cell, I have considered 39 atoms (733 733733$)$ of the $(A B C A B C)$ 


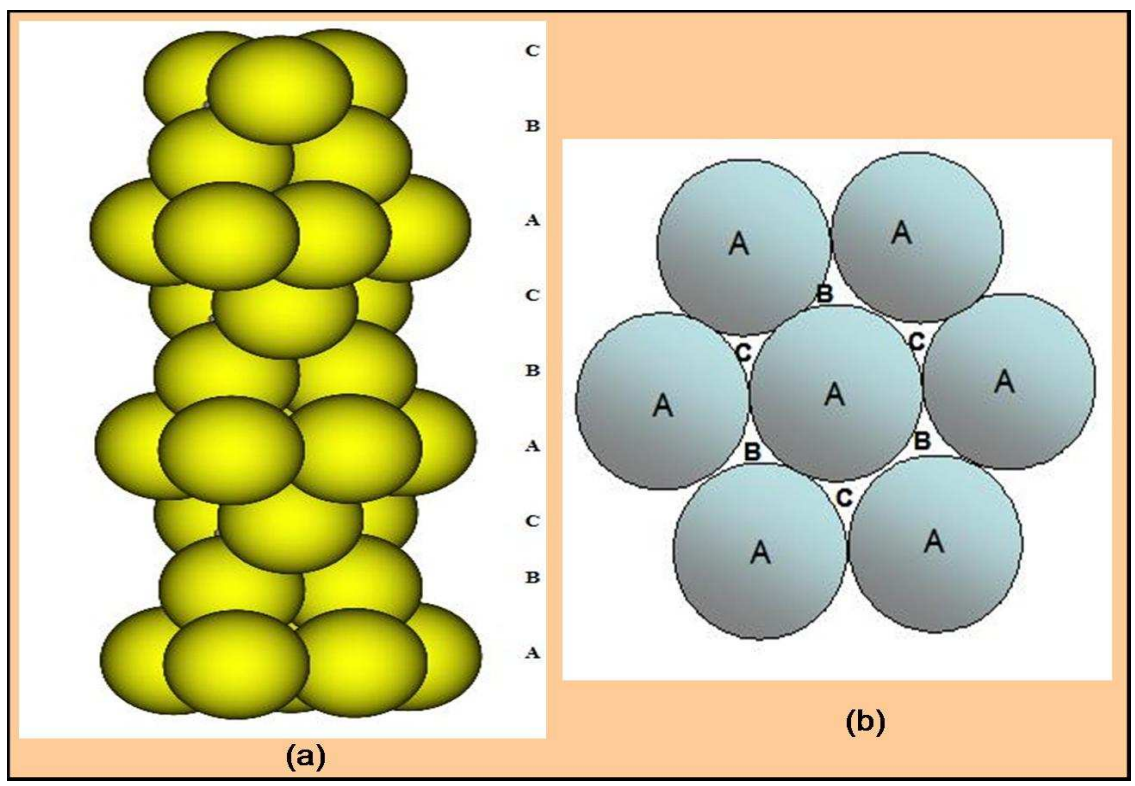

Figure 3.1: $A B C A B C A B C$ stacking of the $f c c F e$; (a) side view, (b) top view used to construct the Fe-nanowire

periodic series. The Lattice parameter along the wire axis was $18.18 \AA$ A The other two sides of the unit cell were taken to be $15 \AA$ to ensure negligible interaction between the nanowire with its image along the $\mathrm{x}$ and $\mathrm{y}$ direction. Then $\mathrm{Fe} / \mathrm{Pt} / \mathrm{Fe}$ multilayered nanowire is constructed by replacing Iron atoms layer wise with Platinum atoms. It should be noted that the Platinum is also $f c c$ in bulk phase with lattice parameter of $3.92 \AA$ [37].

VASP code [39], which is based upon the plane wave basis set and uses ultra-soft pseudo potential, is used to describe the valance core interaction. The density functional theory within the local spins density functional approximation (LSDA) [35], discussed in details in Chapter II is used for my calculations. To test the robustness of LSDA, I have tested this approach for bulk $f c c \mathrm{Fe}$. The calculated cohesive energy per atom and magnetic moment per atom in fcc iron is summarized in Figure. 3.2 


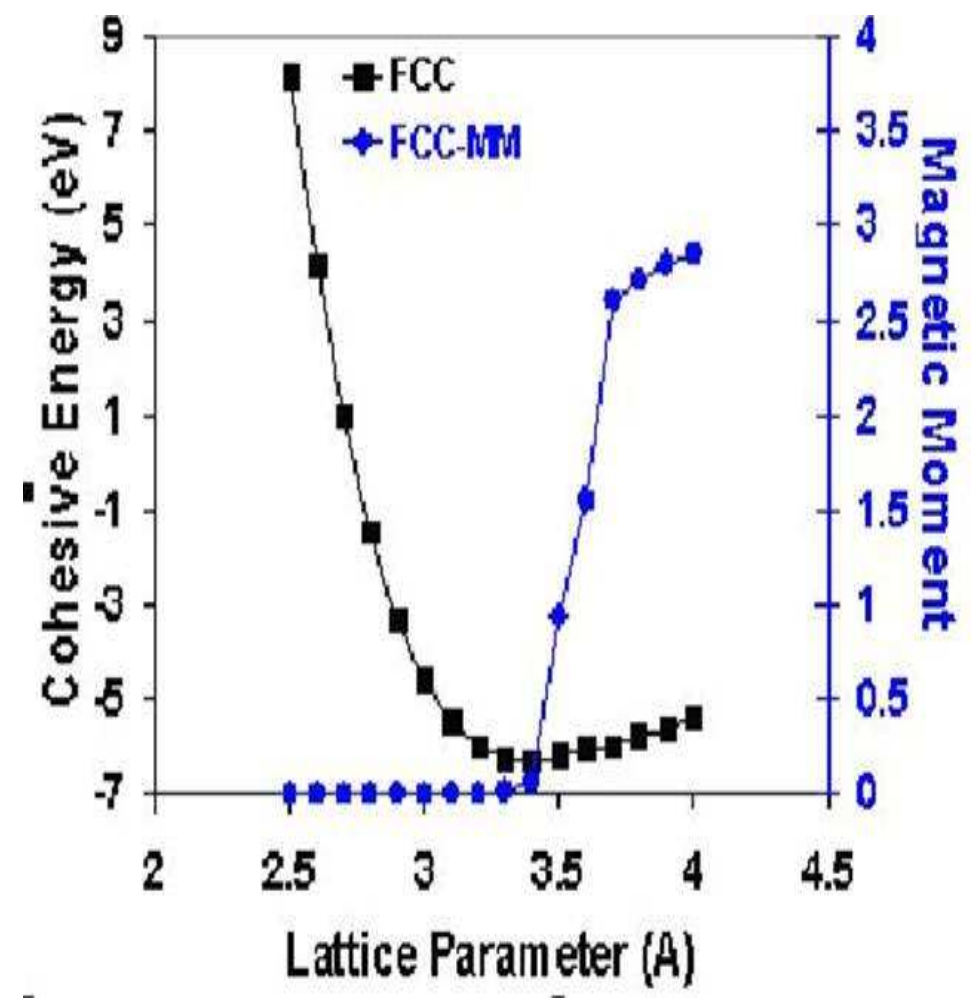

Figure 3.2: Calculated cohesive energy, $E_{c}$,(black line), and magnetic moment(blue line) per Iron atom as a function of lattice parameter in fcc solid, obtained using LSDA approximation

The equilibrium lattice constant of pure iron in $f c c$ phase is found to be $3.4 \AA$, which is in good agreement with the reported value of $3.57 \AA[36]$. The ground state $f c c$ structure is found to be paramagnetic with $0 \mu_{B}$ magnetic moment. This agrees with the previously reported results [36]. It should be noted that the gradient corrected approach (GGA) found to overestimate the magnetism for the fcc Fe in the ground state as shown in Figure. 3.3

Thus, we have not used GGA approach for our calculations. The robustness of the LSDA approach for the bulk $P t$ has also been tested. The calculated cohesive energy as a function of lattice parameter is summarized in Figure. 3.4. The equilibrium lattice parameter is 


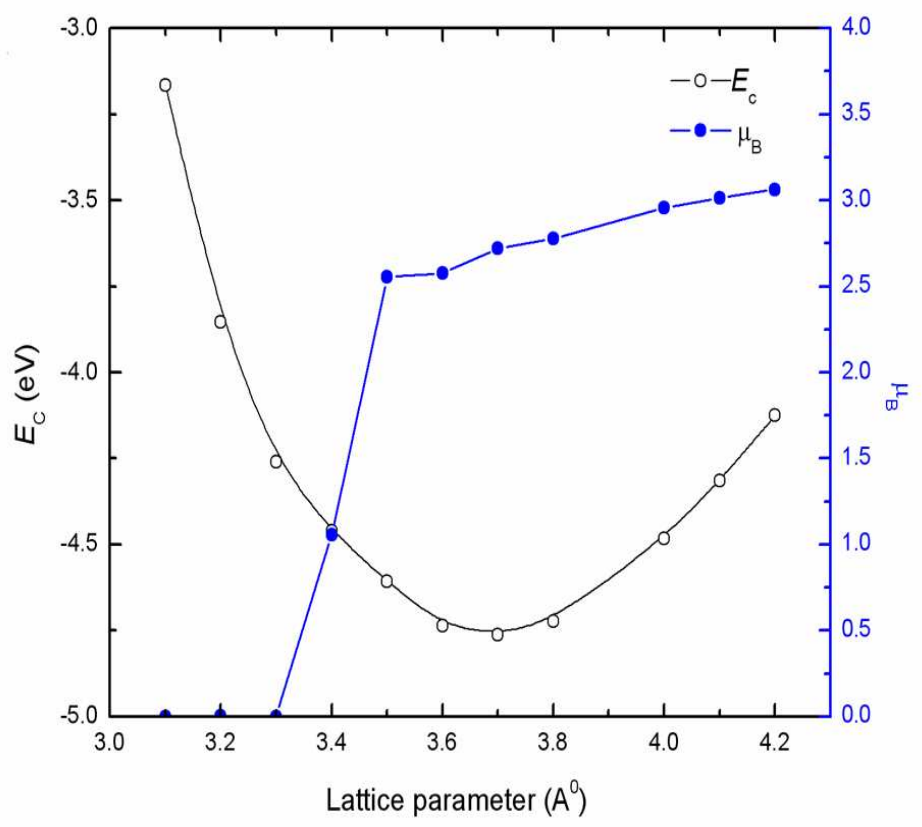

Figure 3.3: Calculated cohesive energy, $E_{c}$,(black line), and magnetic moment(blue line) per Iron atom as a function of lattice parameter in fcc solid, obtained using GGA approximation

found to be $3.92 \AA$, which is in very good agreement with the reported equilibrium lattice parameter of $3.9 \AA$ for $P t$ [37].

The $1 \times 1 \times 1 k$-point mesh within the Monkhorst-Pack scheme is used for geometrical structure optimization of the nanowire. During structural relaxation, the minimum force on each atom is chosen to be $0.01 \mathrm{eV} / \mathrm{A}$. The convergence threshold for energy was taken to be $10^{6} \mathrm{eV}$. Larger $k$-point mesh ( $\left.1 \times 1 \times 11\right)$ for sampling the Brillouin zone is used for calculating the electronic and magnetic properties of the optimized nanowires. The plane wave cutoff was taken to be $237.6 \mathrm{eV}$ and kept fixed for all the multilayered nanowires. I have used Wigner-Seitz radii of 2.46 a.u. and 2.75 a.u. for iron and platinum respectively 


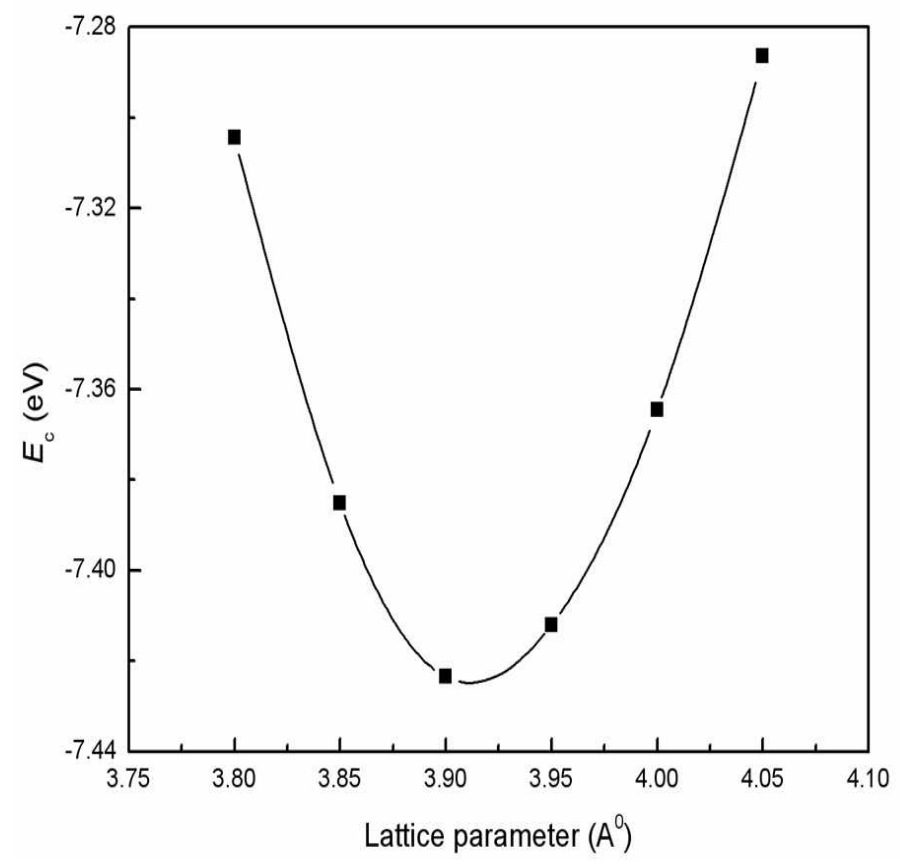

Figure 3.4: Calculated cohesive energy, $E_{c}$, per Platinum atom as a function of lattice parameter in fcc solid, obtained using LSDA approximation

to calculate the local magnetic moment of individual atom in multilayered nanowire. This calculation is based upon the collinear magnetism. Since the dimension of the considered system is quite small, the nanowire is assumed to consist of single magnetic domains.

\subsection{Results and discussions}

The results are organized as follows. First, the structure and stability are discussed in Sec. 3.3.1 and the magnetic properties in Sec. 3.3.2. This is followed by Sec. 3.3.3, which deals with the spin polarized density of states, and Sec. 3.3.4 describes the spin polarized band structure. In Sec. 3.3.5, the results of $\mathrm{Fe} / \mathrm{Pt}$ system are compared with $\mathrm{Ni} / \mathrm{Al}$ and $\mathrm{Ni} / \mathrm{Cu}$ 
nanowire systems.

\subsubsection{Structure and stability}

The geometry optimizations for pristine as well as multilayer nanowires were performed using the procedure discussed in Chapter II. In the pristine $\mathrm{Fe}$-nanowire, a significant atomic structure relaxation in the $A$ plane of the $\mathrm{ABCABCABC}$ unit cell was noticed as can be observed in Figure. 3.5 In the $\mathrm{Fe} / \mathrm{Pt}$ multilayer nanowire, the structural relaxation in the radial outward direction is noticeable in the $P t$ part of the wire as presented in the Figure. 3.5. This is expected because the lattice parameter for bulk $P t$ is $\sim 0.4 \AA$ larger than that of $f c c F e$. Next, we discuss the stability of these nanowires.

Stability: To infer the stability, the cohesive energy, $E_{c}$, per atom in each wire is calculated using the formula:

$$
E_{c}=\frac{\left(E_{N W}-\sum_{i} E_{i}\right)}{N}
$$

$E_{N W}$ is the energy of the nanowire, $\mathrm{N}$ is the number of atoms in the unit cell, and $E_{i}$ corresponds to the energy of the isolated atom. The results for calculated cohesive energy are summarized in Figure. 3.6

It can be inferred from this figure that the cohesive energy increases with the increase in the number of platinum spacer layers. Thus the platinum rich nanowires are more stable. Here, 


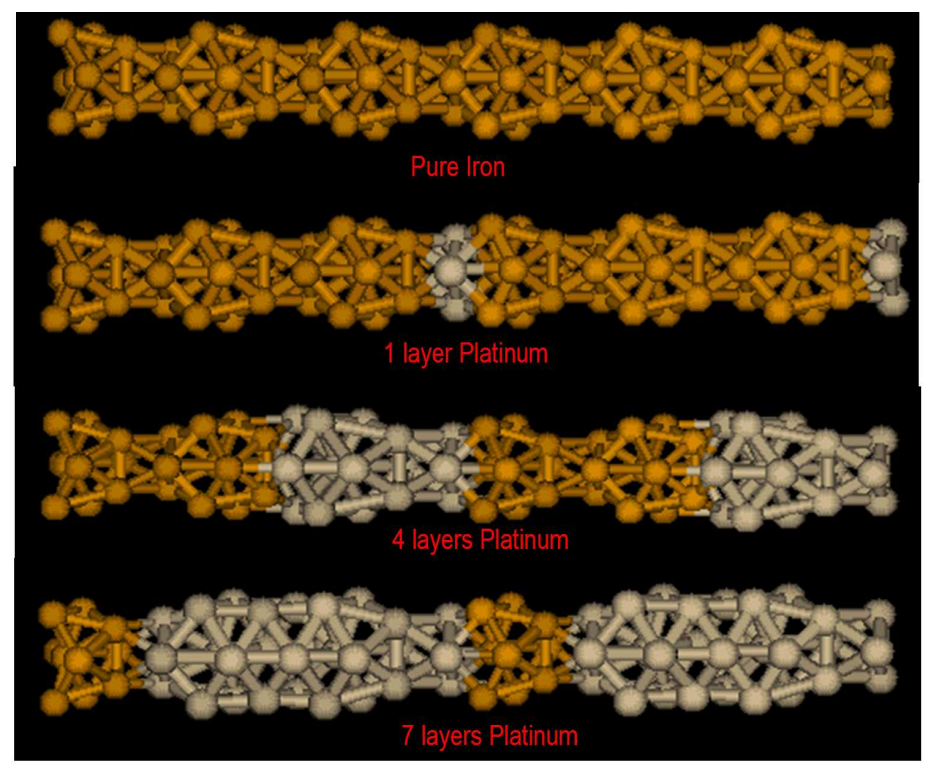

Figure 3.5: Optimized (a) Fe39; (b) Fe36Pt3; (c) Fe23Pt16; and (d) Fe10Pt29nanowire structures. Notation: golden yellow (Fe); silver white (Pt). Two unit cells for each structure are presented for better visualization. Reprinted figure with permission from, Puspamitra.Panigrahi, Ranjit.Pati, Phys. Rev. B. 76, 024431(2007), (C)(2007) The American Physical society.

the $N(P t)=0$ point corresponds to the pristine $F e$ nanowire of $\sim 0.4 \mathrm{~nm}$ diameter. The calculated cohesive energy of the pristine $F e$ wire is found to be $4.7 \mathrm{eV}$, i.e $\sim 1.7 \mathrm{eV}$ less stable than the bulk Iron in $f c c$ phase. It suggests that the stability of the system decreases with reduced coordination. The magnetic properties of this system are elucidated in the following section. 


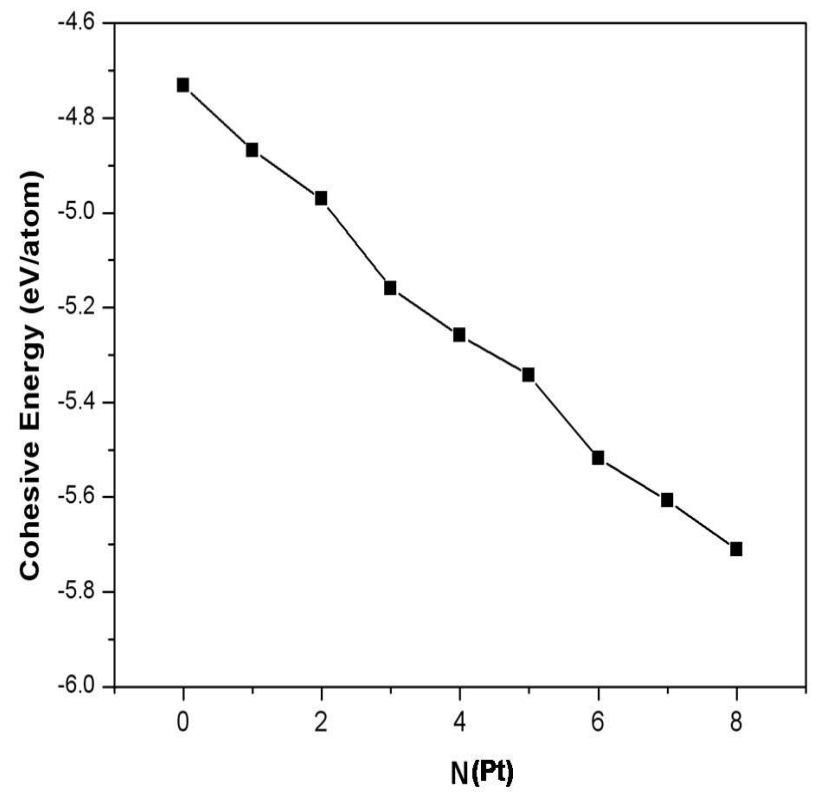

Figure 3.6: Calculated cohesive energy, Ec, as a function of the number of Pt spacer layers, $N(P t)$, in the nanowire structures. Reprinted figure with permission from Puspamitra.Panigrahi, Ranjit.Pati, Phys. Rev. B. 76, 024431(2007), (C)(2007)

The American Physical society

\subsubsection{Magnetic Properties}

Magnetic moment: The average magnetic moment per iron atom was calculated as

$$
\mu_{a v}=\frac{\sum \mu(F e)}{N_{a t o m s}(F e)}
$$

$\sum \mu(\mathrm{Fe})$ represents the sum of individual magnetic moments of $\mathrm{Fe}$ atoms in the wire; $N_{\text {atoms }}$ corresponds to the total number of $\mathrm{Fe}$ atoms in the Iron part of the barcode nanowire. 
The results for the calculated magnetic moment as a function platinum spacer thickness, $N(P t)$, are presented in Figure. 3.7 It can be noted that the magnetic moment per atom monotonically increases with the increase in the number of non-magnetic platinum spacer layers. The non-uniform feature observed in Figure. 3.7 is due to the non-uniform increase in number of platinum atoms with the increase in the number of platinum layers.

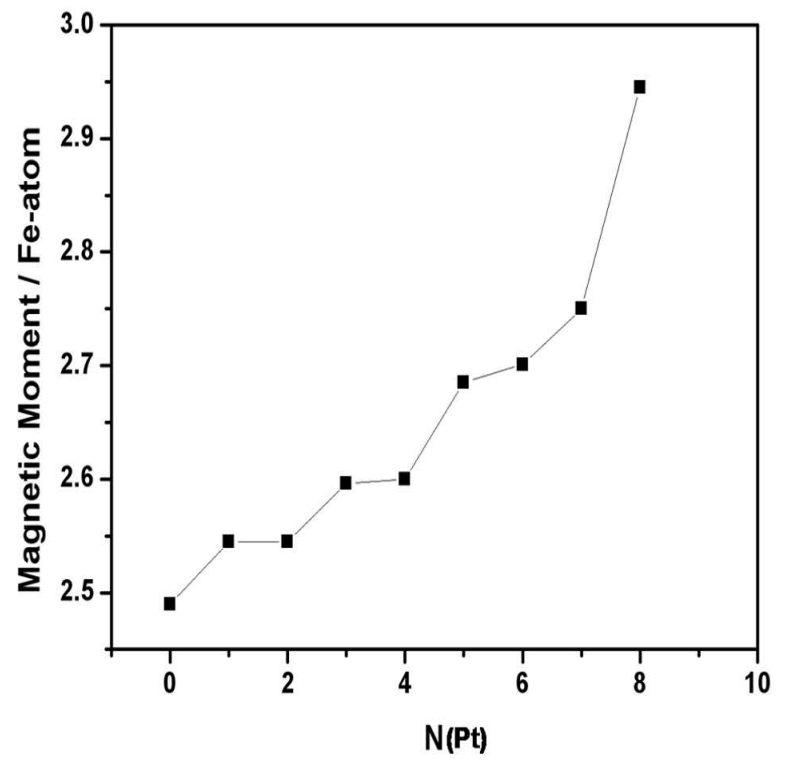

Figure 3.7: Calculated magnetic moment per Fe atom $\left(\mu_{a v}\right)$ as a function of the number of Pt spacer layers, $N(P t)$. Reprinted figure with permission from Puspamitra.Panigrahi, Ranjit.Pati, Phys. Rev. B. 76, 024431(2007), (C)(2007) The American Physical society

For example, $N(P t)=1$ represents increase in one spacer layer with three $P t$ atoms in the system and the trend continued for $N(P t)=2,4,5,7$ and 8 each time by replacing three $P t$ atoms with three $F e$ atoms in the 39 atom unit cell, whereas at $N(P t)=3$ and 6 , there is an increase of seven $P t$ atoms in the nanowire. This explains the observed non 
uniform increase in magnetic moment. The average magnetic moment per atom in the pristine iron nanowire is found to be $2.49 \mu_{B}$ compared to the paramagnetic $f c c$ bulk Iron in the ground state. A very similar trend of increase in magnetic moment $\mu_{a v}$ of Fe with the increase of Platinum concentration was reported by Podgorny [62] in FePt bulk alloy system. It was found that the $\mu_{a v}$ of $\mathrm{Fe}$ increases from $2.54 \mu_{B}$ in $\mathrm{PtFe}_{3}$ alloy through 2.84 $\mu_{B}$ in $\mathrm{Pt}_{2} \mathrm{Fe}_{2}$ to $3.28 \mu_{B}$ in $\mathrm{Pt}_{3} \mathrm{Fe}$ in the FM phase. To understand the origin of enhanced magnetic moment for $\mathrm{Fe}$ atom, the individual magnetic moment associated with $\mathrm{Fe}$ and $\mathrm{Pt}$ atoms were analyzed in the respective nanowire. For a clear understanding the individual magnetic moment for the first seven atoms which are the most affected inter-facial $\mathrm{Fe}$ atoms, referred as the A layer atoms from the $\mathrm{ABCABCABC}$ series, are presented for the four representative nanowires in Table 3.1.

Table 3.1: Comparison of individual magnetic moment (in the unit of $\mu_{B}$ ) of $\mathrm{Fe}$ atoms in $\mathrm{Fe}_{39}$ (pristine)nanowire with that of the corresponding $\mathrm{Fe}$ atoms in $\mathrm{Fe}_{36} \mathrm{Pt}_{3}$ (1-Layer Pt), $\mathrm{Fe}_{23} \mathrm{Pt}_{16}$ (4-Layer Pt), $\mathrm{Fe}_{10} \mathrm{Pt}_{29}$ (7-Layer Pt) multilayered nanowires

\begin{tabular}{lcccl}
\hline Atom & $\begin{array}{c}\text { Pristine } \\
\mathrm{N}(\mathrm{Pt})=0\end{array}$ & $\begin{array}{c}\text { 1-Layer Pt } \\
\mathrm{N}(\mathrm{Pt})=1\end{array}$ & $\begin{array}{c}\text { 4-Layer Pt } \\
\mathrm{N}(\mathrm{Pt})=4\end{array}$ & $\begin{array}{l}\text { 7-Layer Pt } \\
\mathrm{N}(\mathrm{Pt})=7\end{array}$ \\
\hline $\mathrm{F} 1$ & 1.01 & 1.29 & 1.52 & 1.68 \\
$\mathrm{Fe} 2$ & 2.74 & 2.87 & 2.94 & 3.03 \\
$\mathrm{Fe} 3$ & 2.74 & 2.80 & 2.79 & 2.85 \\
$\mathrm{Fe} 4$ & 2.74 & 2.80 & 2.80 & 2.84 \\
$\mathrm{Fe} 5$ & 2.74 & 2.87 & 2.94 & 3.03 \\
$\mathrm{Fe} 6$ & 2.74 & 2.80 & 2.78 & 2.83 \\
$\mathrm{Fe} 7$ & 2.74 & 2.87 & 2.94 & 3.03 \\
& & & & \\
\hline
\end{tabular}

From Table 3.1, one could see a steady monotonic increase in the magnetic moment with 
the decrease in $N(F e)$ or increase in $\mathrm{N}(\mathrm{Pt})$. It is found that the change is more substantial for the core Iron atom $(\mathrm{Fe} 1)$ as compared to the peripheral $\mathrm{Fe}$ atoms. It is evident from the inter-facial structure of $\mathrm{Fe} / \mathrm{Pt}$ nanowire that the core iron atom has larger number of $P t$ nearest neighbors than the peripheral $\mathrm{Fe}$ atoms resulting in maximum increase in its magnetic moment compared to the peripheral $\mathrm{Fe}$ atoms. Now, the question arises: What is the cause for this increase in magnetic moment with increase of nonmagnetic Platinum spacer layer thickness? Magnetic moment arises due to the difference between spin up and spin down population in the system. Thus, to get a clear understanding of this trend, the up spin and down spin populations of $\mathrm{Fe}$ atoms both in $\mathrm{Fe} / \mathrm{Pt}$ and in pristine nanowire were analyzed. Based on the analysis, a new mechanism is proposed [67].

Mechanism for magnetic moment enhancement: The increase in magnetic moment of the iron atoms at the $\mathrm{Fe} / \mathrm{Pt}$ interface is found to be due to spin flip as well as multi-step electron transfer process. To give a schematic view of this proposed mechanism, the nanowire with four layers of platinum spacer is illustrated exclusively as an example. A substantially more increase in spin up population of the $\mathrm{Fe}$ atom at the $\mathrm{Fe} / \mathrm{Pt}$ interface as compared to the increase in spin down population was found from the spin population analysis. This illustration is depicted in Figure. 3.8. The increase in up and down spin population of iron layer $(F e \mathrm{I})$ is due to both spin up and spin down electron transfer from the nearest interface platinum layers. This would, in fact, suggest negative magnetic moments at the interface Platinum atoms $(P t \mathrm{I})$. However, it is found that the interface Platinum atoms have positive magnetic moment. Analyzing the spin population of the Platinum layers, it is evident that 
those layers away from the interface have substantially very small magnetic moment. This suggests that the electron transfer occurs from the second Platinum interface layer (PtII) to the first Platinum (PtI) interface layer. This could again lead to negative magnetic moments in the Platinum atoms at the second Platinum interface (Pt II) which is also not happening. Rather there is an increase in Platinum magnetic moment at the $\mathrm{Fe} / \mathrm{Pt}$ interface $(\mathrm{PtI})$ as compared to that of (PtII). This is due to flipping of few spin down electrons due to the strong magnetic interaction with the interface iron layer that has a large positive magnetic moment. And the atoms at the second interface (PtII) have a smaller positive magnetic moment compared to that of $(P t \mathrm{I})$ is only due to multi-step electron transfer process. Further depending upon the thickness of the Platinum spacer layer, there is always a competition between spin flip and multi-step electron transfer process. For example, in the case of eight platinum spacer layers, it is found that the spin up population increases and the spin down population decreases for the $\mathrm{Fe}$ atoms at the $\mathrm{Fe} / \mathrm{Pt}$ interface as compared to that for the corresponding $\mathrm{Fe}$ atoms in the pristine nanowire. These larger difference in the spin up and spin down populations leads to an increased magnetic moment for the inter-facial $\mathrm{Fe}$ atoms. In this case there is a substantial polarization of Platinum atoms in the inter-facial layer resulting in a positive magnetic moment for them, whereas the Platinum layers away from the interface shows a negative magnetic moment. Here we proposed the multi-step electron transfer process playing a more dominant role in modulating the magnetic property at the interface. Further examination of the individual magnetic moments suggests the magnetic moments of the Fe, which are far away from the $\mathrm{Fe} / \mathrm{Pt}$ interface virtually remains same 
as that in pristine iron nanowire. However, there is a strong electron exchange interaction between $\mathrm{Fe}$ and $P t$ at the interface which induces positive magnetic moments on $P t$ layers. The effect diminishes for the $P t$ layers that are away from the interface. Similar effect of charge transfer is also found in $\mathrm{Fe} / \mathrm{Pt}$ bulk system [62]. From a careful inspection of the $\mu_{a v}$ from Figure. 3.7, the average magnetic moment per iron atom found to increase monotonically with a $\sim \frac{1}{N(F e)}$ dependence, where $N(F e)=9-N(P t) ; N(P t)$ is the number of $P t$ layers in the multilayered nanowires.

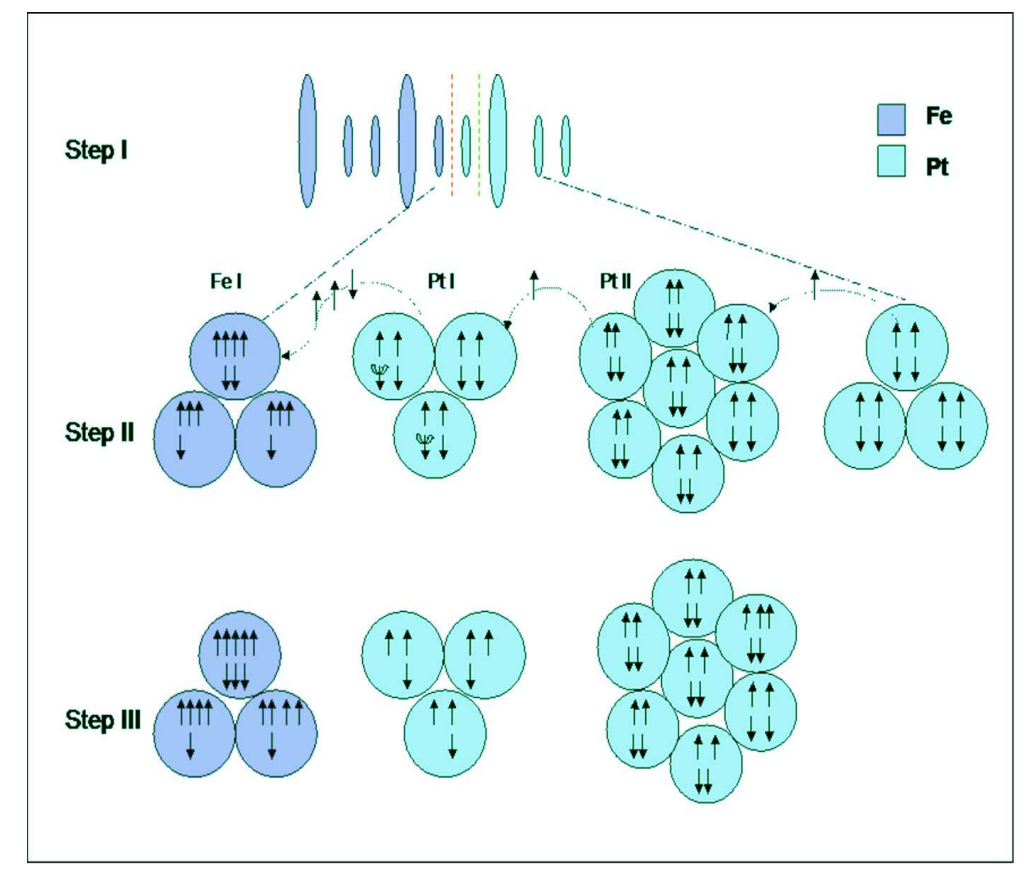

Figure 3.8: Schematic representation of spin flipping and multistep electron transfer process in $\mathrm{Fe}_{23} \mathrm{Pt}_{16}$ barcode wire

The number ' 9 ' comes from the total number of layers in the 39 atoms unit cell. To understand this approximate $\frac{1}{N(F e)}$ behavior of the $\mu_{a v}$, we have analyzed the individual magnetic moments associated with the $\mathrm{Fe}$ and $P t$ atoms in the $\mathrm{Fe} / \mathrm{Pt}$ nanowires. With an aim to ex- 
trapolate this mechanism further for a longer nanowire with large number of spacer layers, a model is proposed to explain the $\frac{1}{N(F e)}$ dependency of $\mu_{a v}$ of $F e$ in the next Section. Model for magnetic moment variation : Since the introduction of $F e / P t$ interface increases the local magnetic moment of the interfacial $F e$ atoms compared to that in pristine $\mathrm{Fe}$ nanowire, one could write the average magnetic moment per iron atom in $\mathrm{Fe} / \mathrm{Pt}$ hybrid nanowire as:

$$
\mu_{a v}=\mu_{a v}(\text { pristine })+d \mu_{a v}
$$

$\mu_{a v}$ (pristine) is the average magnetic moment per iron atom in pristine $\mathrm{Fe}_{39}$-nanowire. The average contribution from the interface structure can be approximated as:

$$
d \mu_{a v}=\frac{N_{F e-P t}}{N_{F e-a t o m s}}
$$

$N_{F e-P t}$ is the number of nearest neighbor $\mathrm{Fe}$ atoms of the $P t$ interface and $N_{F e-a t o m s}$ is the total number of $\mathrm{Fe}$ atoms in the barcode wire. Since $\mu_{a v}$ (pristine) is constant for all the representative wires, the variation observed comes from the interface structure. For example, in the studied nanowires with $N(F e)=1,2,3,4,5,6,7,8$; one can find $N_{F e-P t}=14,10,10,14,10,10,14,10 ;$ and $N_{F e-a t o m s}=7,10,13,20,23,26,33,36$. This can be explained as follows. In our 39 atom unit cell (ABCABCABC), for $N(F e)=1$, 
we have one interface between A Iron layer ( 7 atoms) and B platinum layer ( 3 atoms) and another interface between the $\mathrm{C}$ platinum layer ( 3 atoms) and $\mathrm{A}$ iron layer ( 7 atoms) in the next unit cell resulting $N_{F e-P t}=14$. The similar approach can be applied to obtain all elements of $N_{F e-P t}$ series. Calculating the $\mu_{a v}$ from the simple model in eqn. 3.3 and eqn. 3.4 and plotting them as a function of $N(P t)$ Figure. 3.9, we can find a similar trend as shown in Fig. ??. The small discrepancy of the trend between Figure. 3.7 and Figure. 3.9 is attributed to the exact nature of the interfacial electronic structure, which is absent in the simple model eqn. 3.3 and eqn. 3.4

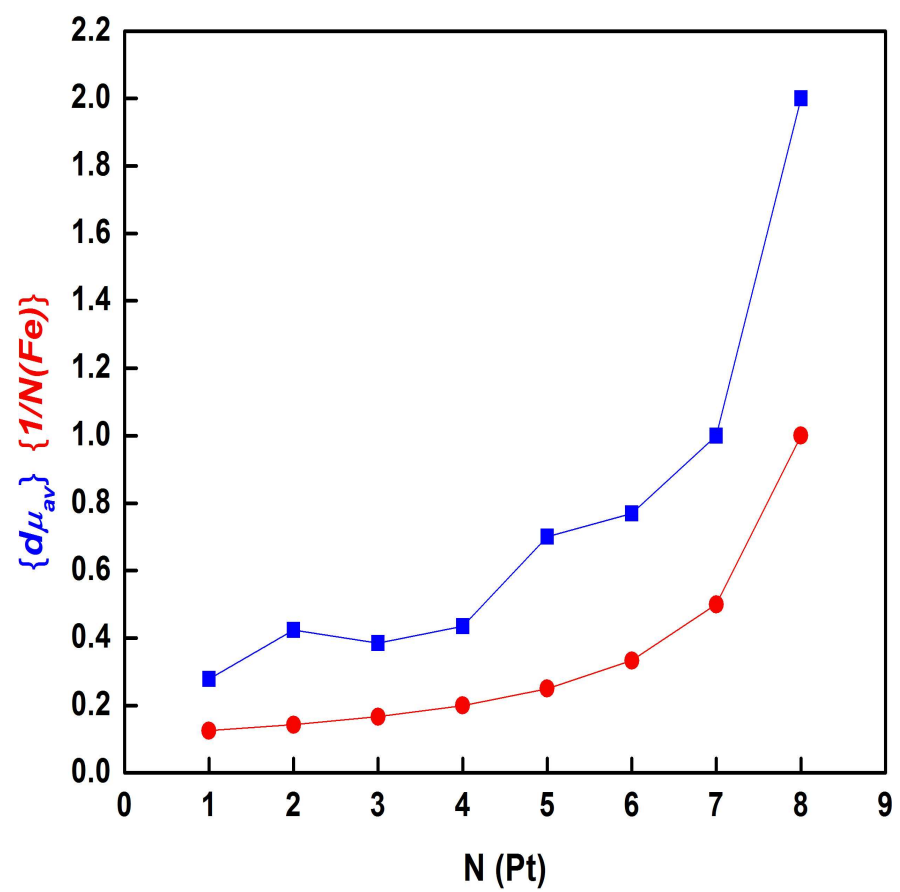

Figure 3.9: Inverse of number of Fe layers $N(F e)$, in the multilayer as a function of the number of Pt spacer layers, $N(P t)$.

Quantum Confinement effect: To understand the confinement effect, the diameter of the pristine $\mathrm{Fe}$-nanowire is increased from $0.4 \mathrm{~nm}$ to $1.1 \mathrm{~nm}$ Figure. 3.10, and the cohesive 
energy and magnetic moment are calculated using the same procedure as for the $\sim 0.4 \mathrm{~nm}$ diameter nanowire. By increasing the diameter of the $F e$-nanowire (for $\sim 1.1 \mathrm{~nm}$ diameter), we found a nearly $1 \mu_{a v}$ reduction in magnetic moment per atom and the stability of the $\sim 1.1 \mathrm{~nm}$ diameter $\mathrm{Fe}$-nanowire was enhanced by $\sim 0.9 \mathrm{eV}$ relative to $\sim 0.4 \mathrm{~nm}$ diameter nanowire. Through a deeper analysis, we found that the surface $F e$ atom in the nanowire has higher magnetic moment of $\sim 2.7 \mu_{B}$ as compared to $1 \mu_{B}$ for a core $F e$ atom. This clearly implies that the reduced coordination number and the confinement effect along the two directions perpendicular to the length of the wire lead to the increase in magnetic moment for the $0.4 \mathrm{~nm}$ diameter $\mathrm{Fe}$-nanowire.

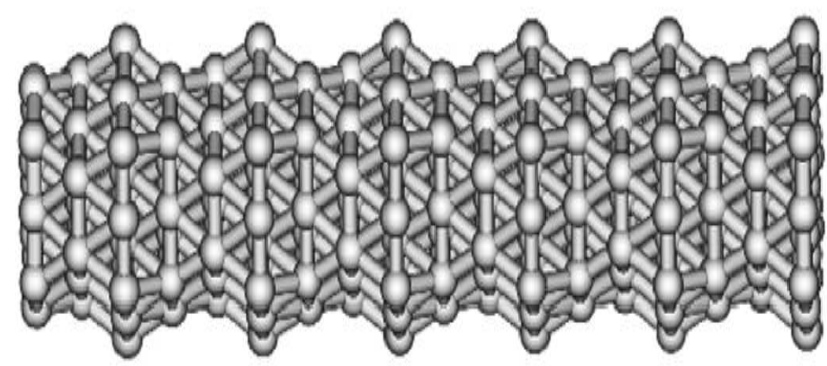

Figure 3.10: Equilibrium $\mathrm{Fe}_{43}$-nanowire structure of $\approx 1.1 \mathrm{~nm}$ diameter.6unit cells are presented for clarity.

\subsubsection{Spin polarized density of states}

To further understand the origin of enhanced stability and increase in the magnetic moment per iron atom in $\mathrm{Fe} / \mathrm{Pt} / \mathrm{Fe}$ hybrid nanowire, the spin polarized $d$-band density of states 
(PDOS) associated with $F e$ and $P t$ were plotted. The PDOS for iron in pristine $F e_{39}$ nanowire Figure. 3.11 shows strong exchange splitting with completely filled spin up $d$ bands. Spin down PDOS is plotted on the negative axis for visualization purposes.

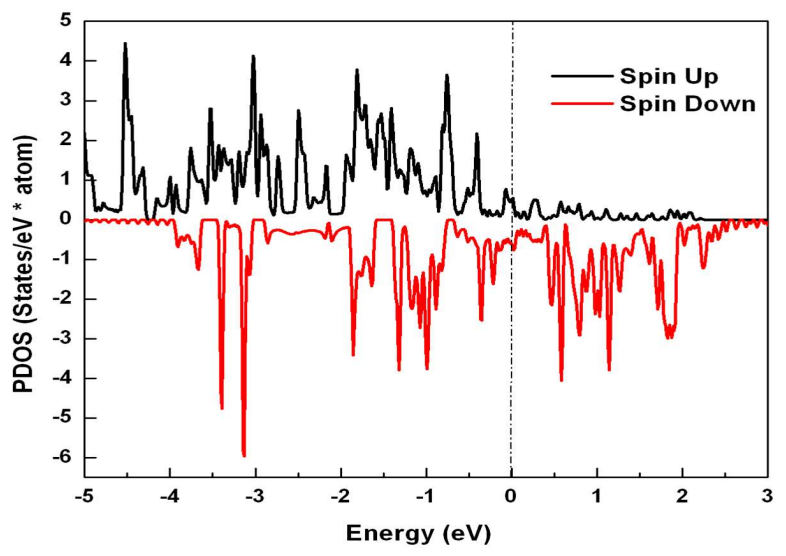

Figure 3.11: Projected spin-polarized d-band density of states (PDOS) of $\mathrm{Fe}$ in $\mathrm{Fe}_{39}$ barcode nanowires. Reprinted figure with permission from, Puspamitra.Panigrahi, Ranjit.Pati, Phys. Rev. B. 76, 024431(2007), (C)(2007) The American Physical society.

In $\mathrm{Fe} / \mathrm{Pt}$ hybrid structure, for example in $\mathrm{Fe}_{23} \mathrm{Pt}_{16}$ nanowire (4 layers of $\mathrm{Pt}$ ), the spin up $d$ bands are again completely filled for $\mathrm{Fe}$ (Figure. 3.12), and the $5 d$ bands for Pt (Figure. 3.13) are almost degenerate with the $3 d$ bands of Fe near the Fermi energy.

From this, a strong hybridization between $\mathrm{Fe}$ and $\mathrm{Pt}$ in $\mathrm{FePt}$ hybrid structure can be interpreted. The stronger hybridization between $F e$ and $P t d$ bands explains the enhanced stability in $\mathrm{Fe} / \mathrm{Pt}$ system. The asymmetry between the spin up and spin down bands of $\mathrm{Pt}$ in Figure. 3.13 explains the polarization of platinum $d$ band due to the exchange interaction. From the structural analysis it is evident that the hybridization between $\mathrm{Fe}$ and $\mathrm{Pt}$ at 


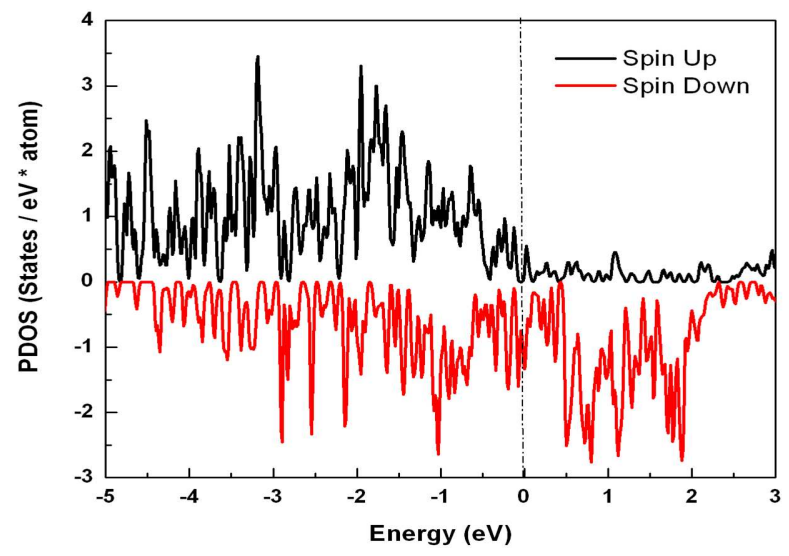

Figure 3.12: Projected spin-polarized d-band density of states (PDOS) of Fe in $F_{23} P t_{16}$ barcode nanowires. Reprinted figure with permission from, Puspamitra.Panigrahi, Ranjit.Pati, Phys. Rev. B. 76, 024431(2007), (c)(2007) The American Physical society.

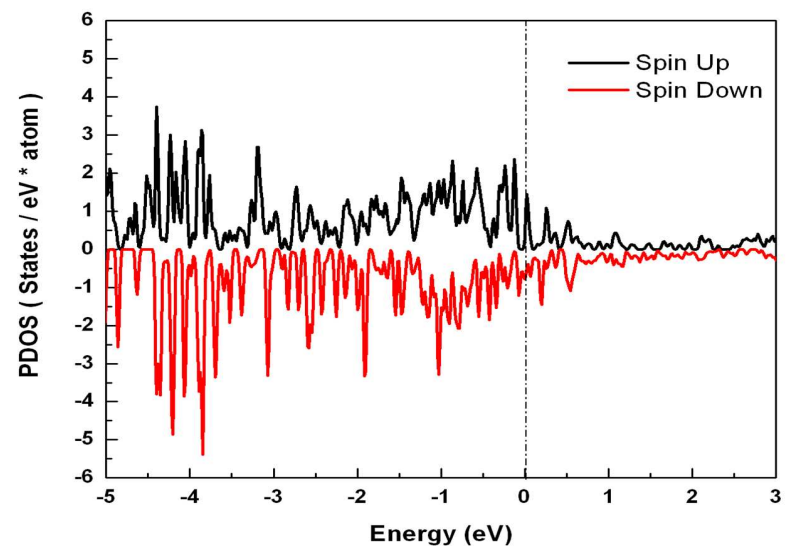

Figure 3.13: Projected spin-polarized d-band density of states (PDOS) of Pt in $\mathrm{Fe}_{23} \mathrm{Pt}_{16}$ barcode nanowires. Reprinted figure with permission from, Puspamitra.Panigrahi, Ranjit.Pati, Phys. Rev. B. 76, 024431(2007), (c)(2007) The American Physical society.

the interface weakens the hybridization between the $3 d$ bands of $F e$-atoms in the nanowire.

This results in a shorter $F e-F e$ bond distance in pristine $F e_{39}$ nanowire by $\sim 0.02 \AA-0.07$ 
$\AA$ compared to that in $\mathrm{Fe} / \mathrm{Pt}$ hybrid system. This increased $\mathrm{Fe}-\mathrm{Fe}$ bond distance leads to band narrowing and enhancement of Iron magnetic moment in $\mathrm{Fe} / \mathrm{Pt}$ hybrid system, especially at the interface.

\subsubsection{Spin polarized energy bands}

The spin-polarized Kohn-Sham energy bands of these nanowires are calculated for understanding the electronic properties. A strong exchange splitting in the band structures is clearly evident in the pristine $\mathrm{Fe}_{39}$-nanowire Figure. 3.14 In the vicinity of the Fermi energy,in the spin up and spin down case, both the valence and conduction bands show a dominant $F e(d)$ character. In the spin up case, the conduction band crosses the Fermi energy whereas in the spin down case, the valence band crosses the Fermi energy. This suggests both the spin up and down channels could contribute to the total conductance

of $G_{0} \approx \frac{2 e^{2}}{h}$ in this wire assuming an ideal transmission, $T \approx 1$ for each spin channel and ignoring spin flip scattering.

In the case of the $\mathrm{Fe}_{36} \mathrm{Pt}_{3}$-nanowire, the spin up valence band near the Fermi energy shows a clear hybridization of $\mathrm{Fe}(d, p)$ bands with the $P t(d, p)$ bands (Figure. 3.15). Where as the conduction bands near the Fermi energy are mostly $\operatorname{FePt}(s, p, d)$ hybrid bands. But as we move away from the high symmetry point, both the valence and conduction bands show dominant $F e(d)$ character. In the spin down case, the valance bands are mainly $F e(d, p)$ 

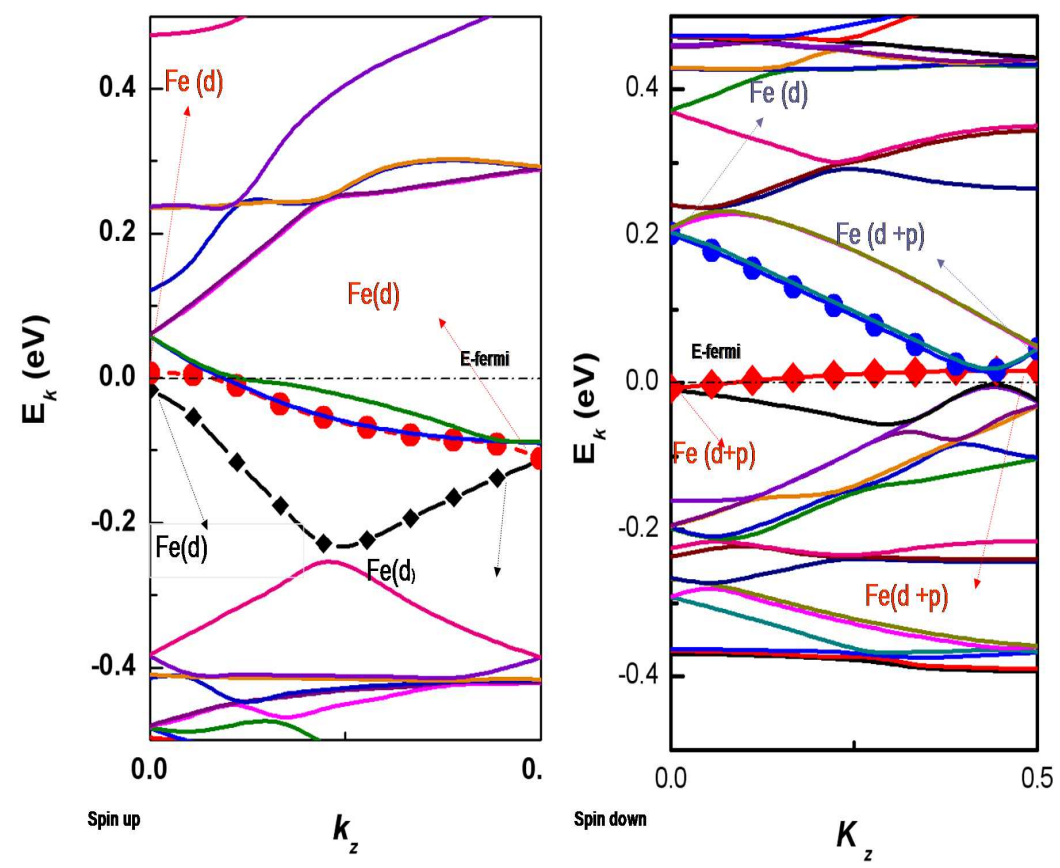

Figure 3.14: Spin polarized electronic band structures for (i)Fe 39 (spin up); (ii) $\mathrm{Fe}_{39}$ (spin down). Reprinted figure with permission from, Puspamitra.Panigrahi, Ranjit.Pati, Phys. Rev. B. 76, 024431(2007), (C)(2007) The American Physical society.

bands and the conduction bands are hybridization of $F e$ and $P t(d, p)$ bands around the Fermi energy. Furthermore, there is a gap opening in the spin down case, and only the spin up conduction band crosses the Fermi energy. Thus only the majority spin band is expected to contribute to the conductance near the Fermi energy, leading to a conductance of $\approx 0.5 G_{0}$.

For the $\mathrm{Fe}_{23} \mathrm{Pt}_{16}$ wire, the spin up conduction bands near the Fermi energy are predominantly $\mathrm{Fe}(s, p)$ bands with a little contribution from $\mathrm{Fe}$ and $\mathrm{Pt}(\mathrm{d})$ hybridization (Figure. 3.16). But the valence bands are mostly $\operatorname{Pt}(d)$ bands. As we move away from the high symmetry point, the conduction bands are mostly $F e$ and $P t(d)$ hybrid bands so also 

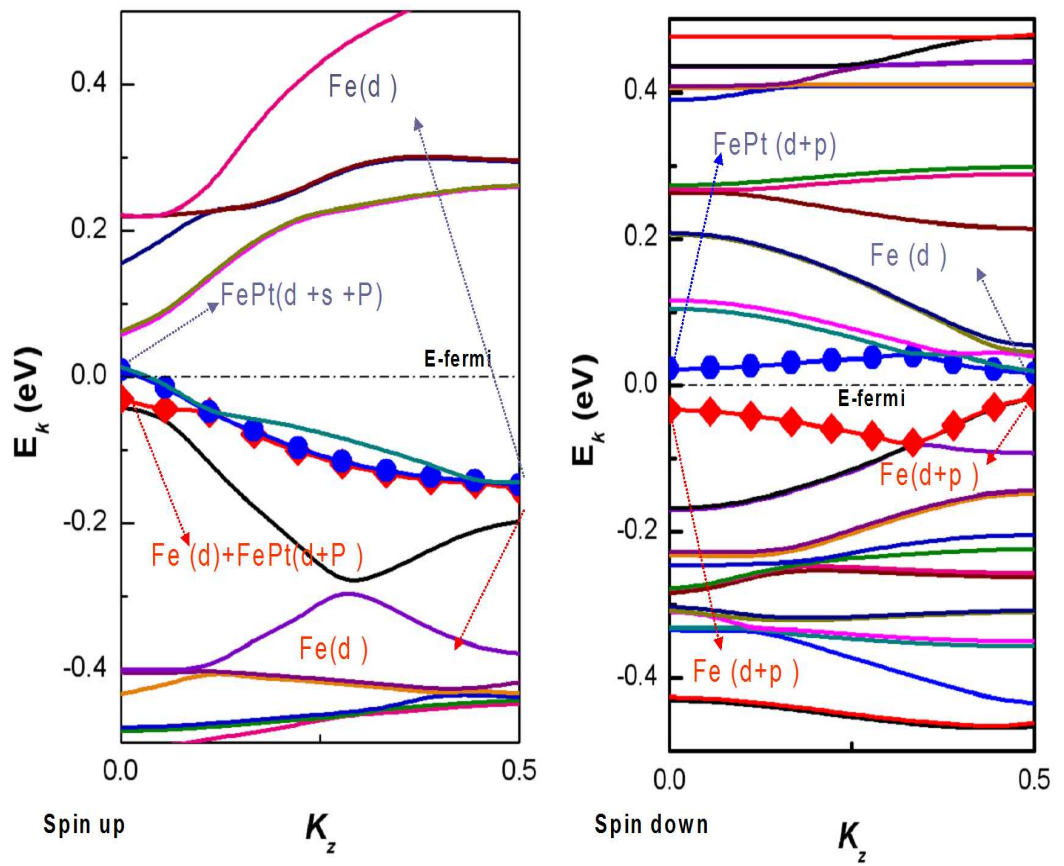

Figure 3.15: Spin polarized electronic band structures for (i) $\mathrm{Fe}_{36} \mathrm{Pt}_{3}$ (spin up); (ii)Fe ${ }_{39} \mathrm{Pt}_{3}$ (spin down).Reprinted figure with permission from, Puspamitra.Panigrahi, Ranjit.Pati, Phys. Rev. B. 76, 024431(2007), (C)(2007) The American Physical society.

the valance bands. In spin down case both valence bands and conduction bands are $F e(d)$ bands, and as we move away from the high symmetry point $P t(d)$ hybrid bands contribute. The observed spin up conduction band crossing the Fermi energy and a gap closure in the spin down channel establishes that both spin up and spin down channels would contribute to the conduction as in the case of pristine $F e_{39}$ nanowire.

In the case of the $\mathrm{Fe}_{10} \mathrm{Pt}_{29}$-nanowire, in the spin up case, both valence and conduction bands show dominant $P t(d)$ character where as in the spin down case both bands are mostly FePt $(d)$ hybrid bands (Figure. 3.17). Also, here one can notice a small gap opening between the spin down conduction and valence bands as compared to that in the $F e_{23} P t_{16^{-}}$ 

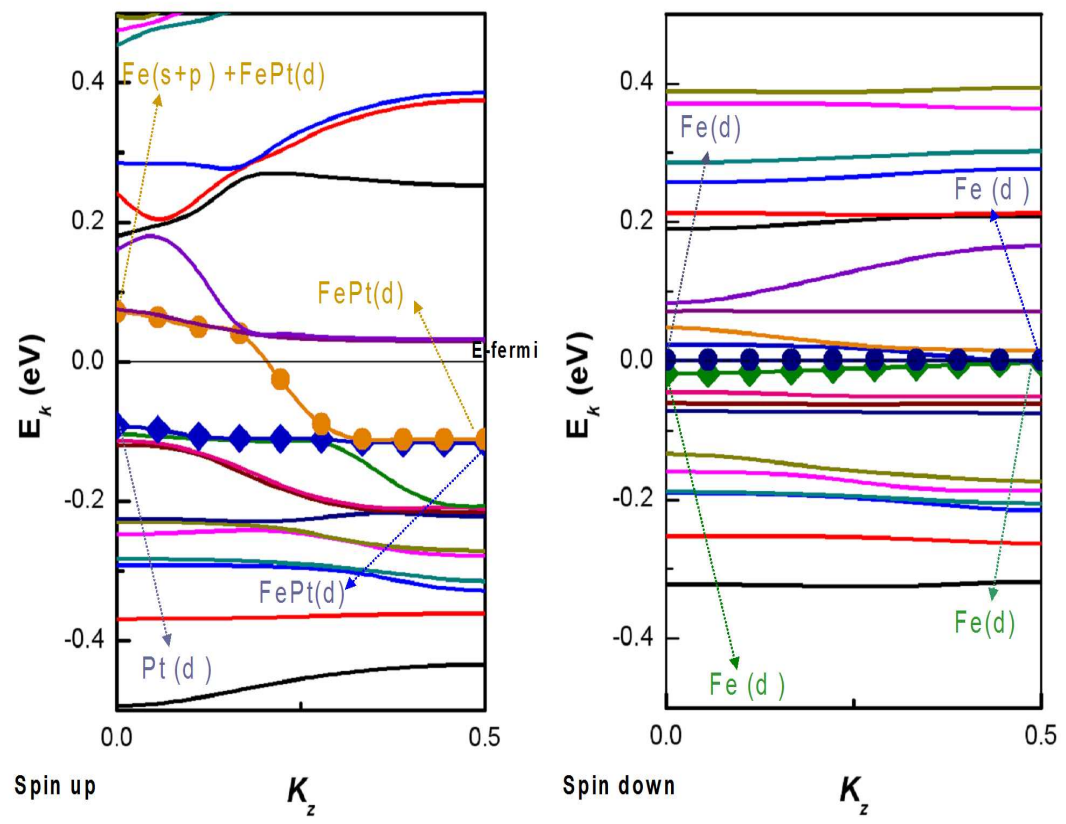

Figure 3.16: Spin polarized electronic band structures for (i) $\mathrm{Fe}_{23} \mathrm{Pt}_{16}$ (spin up); (ii) $\mathrm{Fe}_{23} \mathrm{Pt}_{16}$ (spin down). Reprinted figure with permission from, Puspamitra.Panigrahi, Ranjit.Pati, Phys. Rev. B. 76, 024431(2007), (C)(2007) The American Physical society.

nanowire. This suggests that the spin polarized conductance in these barcode wires can be controlled by controlling the number of $P t$ spacer layers.

Hence, this dependence of spin polarized conductance on the number of Pt layers in the barcode wire confirm the possibilities in controlling magneto-conductance properties of these wires via precise control of the number of ferromagnetic and non-magnetic layer sequences in the wire, which is a prerequisite for their noble applications in magnetoelectronics. 

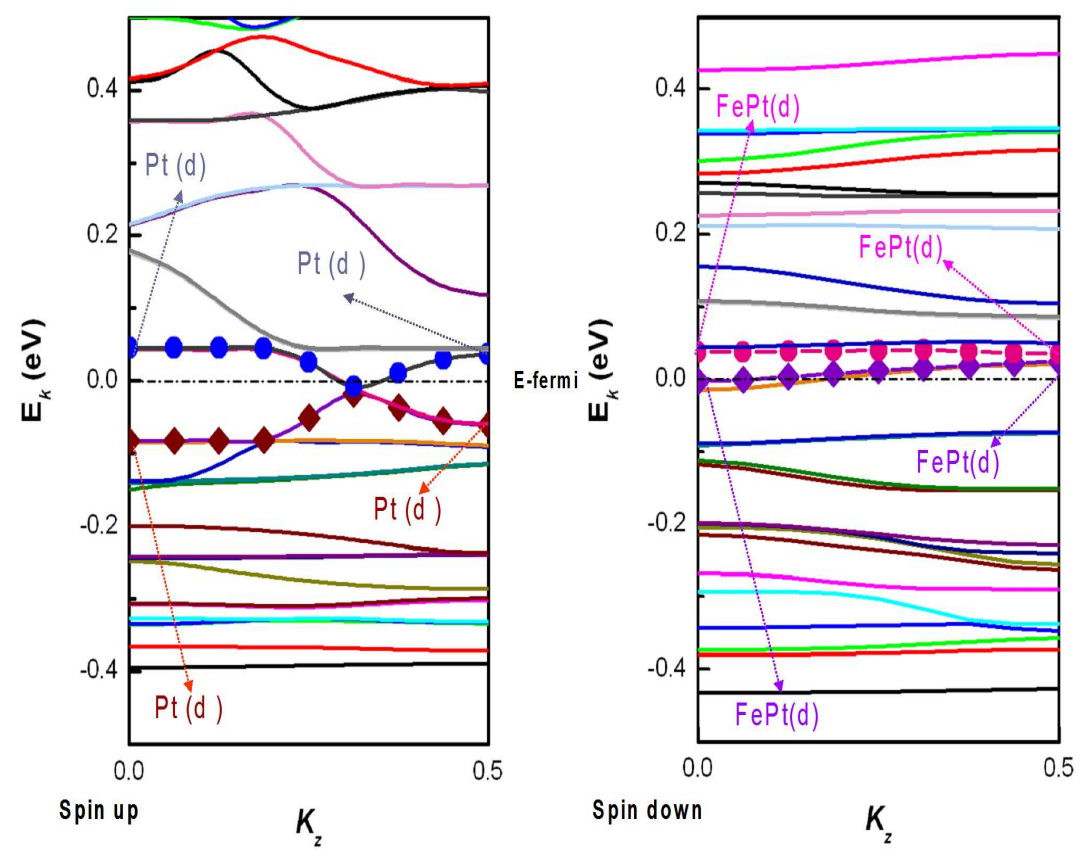

Figure 3.17: Spin polarized electronic band structures for (i) $\mathrm{Fe}_{10} \mathrm{Pt}_{29}$ (spin up); (ii) $\mathrm{Fe}_{10} \mathrm{Pt}_{29}$ (spin down). Reprinted figure with permission from, Puspamitra.Panigrahi, Ranjit.Pati, Phys. Rev. B. 76, 024431(2007), (C)(2007) The American Physical society.

\subsubsection{Comparison with $\mathrm{Ni} / \mathrm{Cu}$ and $\mathrm{Ni} / \mathrm{Al}$ System}

Now the question arises whether the trend observed in the $\mathrm{Fe} / \mathrm{Pt}$ nanowire system discussed before is universal. To answer this question, I have compared my results with the recently published [64] $\mathrm{Ni} / \mathrm{Cu}$ and $\mathrm{Ni} / \mathrm{Al}$ nanowire having similar atomic configurations. Both these systems have equal number of atoms in the unit cell as that of $F e / P t$ system. The new system is with magnetic $\mathrm{Ni}$ nanowire in which the non magnetic $\mathrm{Cu}$ as well as $A l$ was introduced as spacer in a similar fashion as $P t$ was introduced as the spacer in $F e$ nanowire system. Also the structure optimization was done under exactly the same criteria 
and magnetic properties as well as band structure calculation were performed in a similar manner using VASP code. The stability of both the nanowires was calculated as that for $\mathrm{Fe} / \mathrm{Pt}$ nanowire. The stability in both $\mathrm{Ni} / \mathrm{Cu}$ and $\mathrm{Ni} / \mathrm{Al}$ found to increases with increase in the concentration of magnetic $N i$ layers Figure. 3.18, Figure. 3.19. This result is in contrast to that observed increase of stability in $\mathrm{Fe} / \mathrm{Pt}$ nanowire with increase in the concentration of nonmagnetic $\mathrm{Pt}$ layer. The magnetic properties in $\mathrm{Ni} / \mathrm{Cu}$ and $\mathrm{Ni} / \mathrm{Al}$ nanowire showed decreasing trend like a steady decrease of $\mu_{a v}$ with increase of $C u$ spacer layer and a nonmonotonic decrease of $\mu_{a v}$ with $A l$ spacer layer thickness respectively, whereas the $F e / P t$ system showed a monotonic increasing trend in $\mu_{a v}$ with increase of $P t$ spacer layer thickness. The interfacial covalent bonding found to play an important role in reducing magnetic properties in $\mathrm{Ni} / \mathrm{Cu}$ and $\mathrm{Ni} / \mathrm{Al}$ nanowire systems. In $\mathrm{Ni} / \mathrm{Al}$ system, (Figure. 3.20), the directional nature of $N i(d)$ and $A l(p)$ hybridization forces $N i$ to have higher coordination number with $A l$ resulting in a decreasing trend of $\mu_{a v}$. Here the quenching of magnetic moment in $N i$ atom in the $N i / A l$ system is proportional to the number of $A l$ atoms at the $N i-A l$ interface. So in the ABCABC packing series when three atoms $N i$ layer is followed by seven atoms $A l$ layer the quenching effect is more significant compared to seven atoms $N i$ layer followed by three atoms $A l$ layer. The stronger $N i(d)$ and $A l(p)$ hybridization, higher will be the $N i$ coordination number, resulting in decrease in magnetic moment. This explains the non monotonic decreasing trend of magnetic moment in $\mathrm{Ni} / \mathrm{Al}$ nanowire system. Whereas, in $\mathrm{Ni} / \mathrm{Cu}$ system, (Figure 3.21) the strong hybridization between $\mathrm{Ni}(\mathrm{d})$ and $C u(s)$ states, leads to reduction in the number of unoccupied $N i(d)$ down states, which 
results in a decreasing trend of $\mu_{a v}$. In contrast, in the $F e / P t$ system, the spin flip and multi step electron transfer is found to be the main mechanism behind the increasing trend of $\mu_{a v}$ with $P t$ spacer which is discussed at length before.

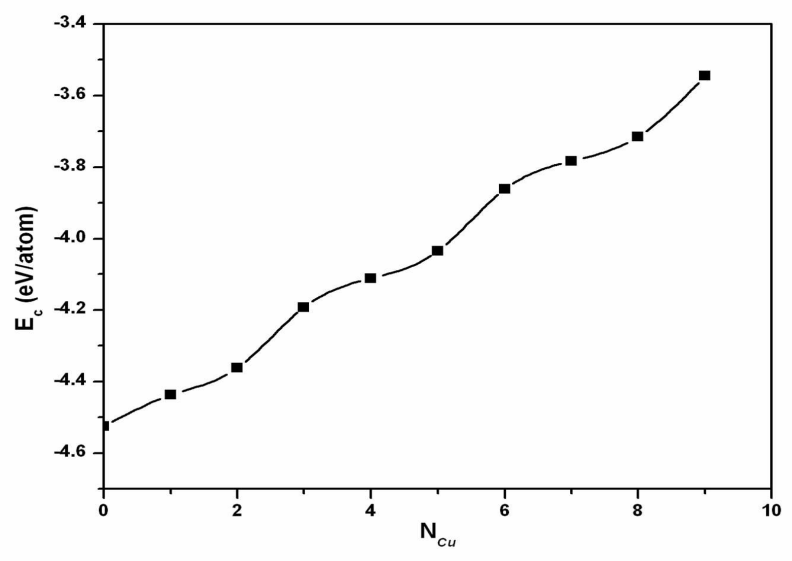

Figure 3.18: Calculated cohesive energy $E_{c}$ as a function of Number of $C u$ layers $\left(N_{C u}\right)$ in $\mathrm{Ni} / \mathrm{Cu}$ multilayered nanowire (Reprinted figure with permission from, Partha.Pratim.Pal, Ranjit.Pati, Phys. Rev. B. 77, 144430(2008), C(2008) The American Physical society: refer Appendix C for permission)

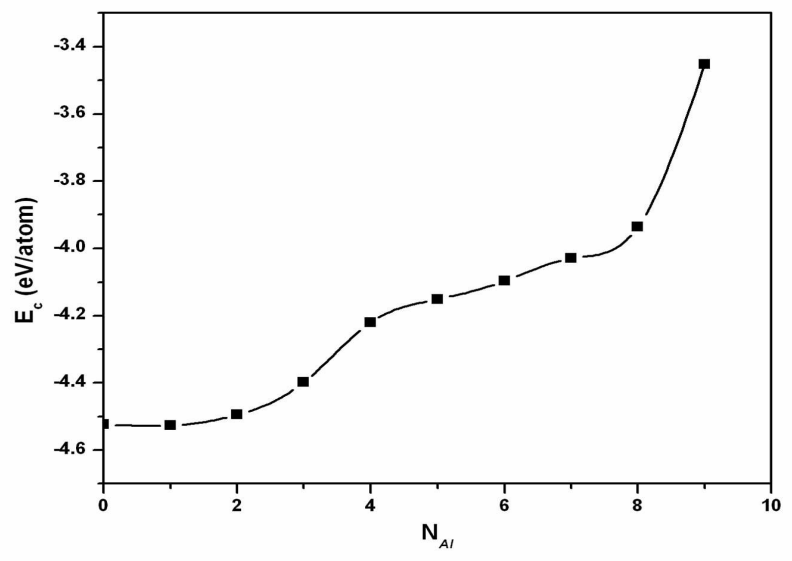

Figure 3.19: Calculated cohesive energy $E_{c}$ as a function of Number of Al layers $\left(N_{A l}\right)$ in $\mathrm{Ni} / \mathrm{Al}$ multilayered nanowire(Reprinted figure with permission from, Partha.Pratim.Pal, Ranjit.Pati, Phys. Rev. B. 77, 144430(2008), C(2008) The American Physical society: refer Appendix C for permission) 


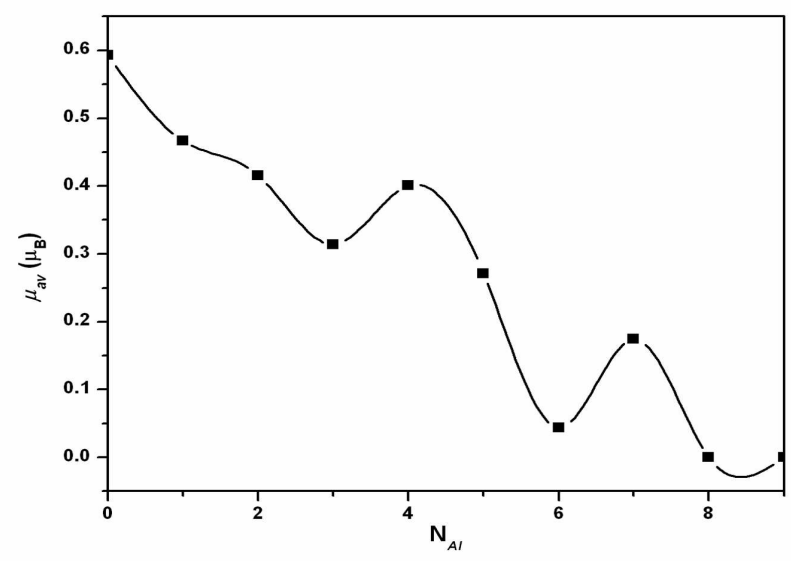

Figure 3.20: Calculated magnetic moment per Ni atom $\left(\mu_{a v}\right)$ as a function of number of Al layers $\left(N_{A l}\right)$ in Ni/Al multilayered nanowire.(Reprinted figure with permission from, Partha.Pratim.Pal, Ranjit.Pati, Phys. Rev. B. 77, 144430(2008), (C)(2008) The American Physical society: refer Appendix C for permission)

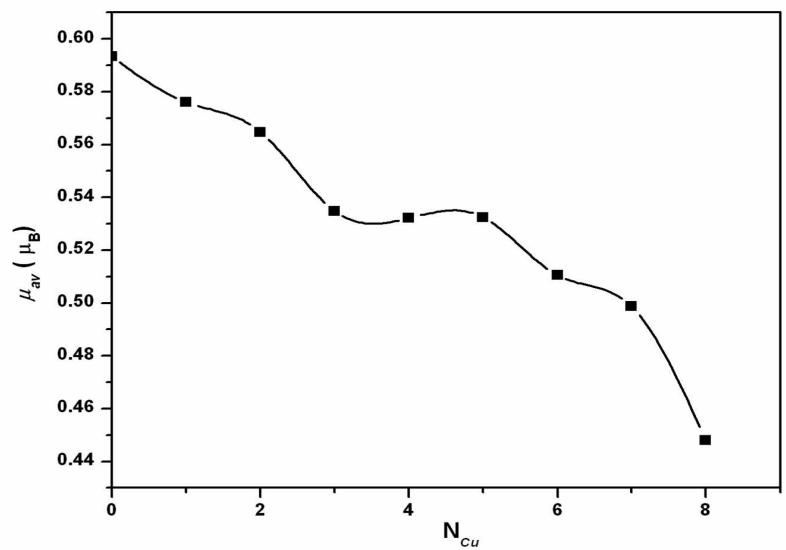

Figure 3.21: Calculated magnetic moment per Ni atom $\left(\mu_{a v}\right)$ as a function of Number of $\mathrm{Cu}$ layers $\left(\mathrm{N}_{\mathrm{Cu}}\right)$ in $\mathrm{Ni} / \mathrm{Cu}$ multilayered nanowire(Reprinted figure with permission from, Partha.Pratim.Pal, Ranjit.Pati, Phys. Rev. B. 77, 144430(2008), (C)(2008) The American Physical society: refer Appendix C for permission) 


\subsection{Summary}

The findings of this present study clearly explain the role of non-magnetic spacer layer thickness in modulating the magnetic and electronic properties of multilayer barcode nanowire. Particularly, our first-principles calculations suggest that increasing the thickness of the $P t$ layer and consequently, reducing the thickness of the $\mathrm{Fe}$ layer in the $\mathrm{Fe} / \mathrm{Pt} / \mathrm{Fe}$ barcode nanowire increases the stability of the nanowire system. Furthermore, this induces a monotonically increasing effect in the average magnetic moment per $\mathrm{Fe}$ atom quantitatively from $2.49 \mu_{B}$ in a pristine $F e_{39}$-nanowire to $2.7 \mu_{B}$ in $F e_{13} P t_{26}$-nanowire and then to $2.95 \mu_{B}$ for $\mathrm{Fe}_{7} \mathrm{Pt}_{32}$-nanowire. This is referred as the barcode layer effect. A simple model based

on the interface structure is proposed which could explain the $\frac{1}{N_{F e}}$ dependence trend in $\mu_{a v}$ obtained from the first-principles density functional calculations. A new mechanism based on the spin flip and multi-step electron transfer process is proposed to explain the enhancement of magnetic moments with the increase of non magnetic spacer layer in $\mathrm{Fe} / \mathrm{Pt}$ system. Thus, the role of interfacial bonding in modulation of the magnetic characters is established thoroughly. The decrease in magnetic moment in a higher diameter nanowire system is also well explained by the quantum confinement effect. Analyzing the spin polarized band structure, a strong dependence of spin-polarized energy bands in the vicinity of the Fermi energy on the non-magnetic layer thickness was also observed. This suggests the potential applications of this nanowire structure in magneto-electronics or spintronics. In the case of $\mathrm{Ni} / \mathrm{Al}$ and $\mathrm{Ni} / \mathrm{Cu}$ system a different trend in magnetic behavior is observed. 
This different magnetic property is attributed to their dissimilar interfacial bonding. For example in $N i / A l$ system the directional character of $N i-A l(d-p)$ hybridization forces $\mathrm{Ni}$ to have higher coordination number with $A l$ resulting in a decreasing but non-monotonic trend in $\mu_{a v}$. In $\mathrm{Ni}-\mathrm{Cu}$, the $s-d$ hybridization reduces the unoccupied $\mathrm{Ni}(d)$ down state leading to a decreasing trend in $\mu_{a v}$. This study again reveals the role of non magnetic spacer in modulating magnetic property but in a dissimilar fashion. So it is evident that by controlling the spacer layer thickness in multilayered magnetic and non magnetic nanowire system, one can tune the ferromagnetic properties. This opens up the possibility for their applications in nanoscale magnetic-barcodes. 


\section{Chapter 4}

\section{Controlling Interlayer Exchange}

\section{Coupling in one dimensional $\mathrm{Fe} / \mathrm{Pt}$}

\section{Multilayered nanowire}

\subsection{Introduction}

The previous chapter dealt with the tuning of ferromagnetic behavior in $\mathrm{Fe} / \mathrm{Pt}$ multilayered nanowire by controlling non-magnetic spacer layer thickness. The magnetic moment of the interfacial $\mathrm{Fe}$ atoms in the $\mathrm{Fe} / \mathrm{Pt}$ multilayered nanowire is found to be higher than that of the $\mathrm{Fe}$ atoms away from the interface. However, the interlayer exchange coupling (IEC) 
needs to be explored in the lower dimensional multilayer nanowire system for their potential application in ultra-high density memory device and magnetic sensors. The magnitude of giant magnetoresistance (GMR) has been found to oscillates with the thickness of nonmagnetic spacer in magnetic/non-magnetic heterostructures. This oscillation in GMR is caused by the alteration in the sign of the interlayer exchange coupling between ferromagnetic layers. Moreover, the exchange coupling is found to oscillate between ferromagnetic and anti-ferromagnetic depending upon the magnetic moment of successive ferromagnetic layers. The exchange coupling $J$, that is obtained from the energy difference between the AFM and FM configurations, corresponds to the strength of the inter layer coupling energy, $J=E_{d}^{\uparrow \downarrow}-E_{d}^{\uparrow \uparrow}$. The quantitative prediction of $J$ and its damped oscillatory behavior with spacer in bulk heterostructures are well explained by the ab initio density functional calculations [9, 10, 11, 12], which is also in good agreement with the experimental results [13, 14, 15]. Though we have started to witness serge in theoretical interest towards multilayered nanowires [68, 69, 70] and tunnel junctions [71] in recent years, only limited calculations have been reported in magnetic nanowires to understand the crucial atomic scale structural heterogeneity at the magnetic/non-magnetic interface and its role on IEC. The ab initio calculations are essential for the accurate determination of exchange energy $J$ for the nanowire systems. To achieve this, one needs to consider both the ferromagnetic (FM) and anti-ferromagnetic (AFM) configuration between the magnetic layers. However, obtaining AFM configuration in nanowire system is a challenging task. At the outset, it is required to explore whether the oscillation of IEC with increase in spacer thickness is 
possible in nanowire system so that the $\mathrm{Fe} / \mathrm{Pt}$ nanowire can be used as a building block in device miniaturization process. In case the answer is in affirmative, whether the RKKY model or the QW model [72, 73, 74, 75] which were able to give a clear explanation for the physics behind the universal oscillation of IEC as a function of spacer in bulk heterostructure, holds good for the multilayer nanowire system. In this project, both FM and AFM coupling between the $F e$ layers for different $P t$ spacer widths are considered and the switching behavior of $J$ is observed while ascending from two to five $P t$ spacer layers. Again increasing the number of spacer layer from five to eight, switching in the sign of $J$ value is observed, but the magnitude of $J$ is found to be significantly smaller. I discuss the oscillatory magnetic properties of Fe/Pt multilayered nanowires as function of Pt spacer width in this Chapter which is organized with the different sections comprising Section 4.2 summarizes the theoretical approach, Section 4.3 presents the results and discussions in details and Section 4.4 describes the summary.

\subsection{Theoretical Approach}

The atomic level structural details are not available a priori for the proposed magnetic nanowires and so the structure determination from the zero score throw up an arduous challenge. The bulk experimental structure was considered as the guiding point for constructing the nanowire structure as described in our previous study [67]. Both $\mathrm{Fe}$ and $P t$ have $f c c$ bulk structures. From the atomic arrangements in the $f c c$ bulk structure, the 
nanowire is constructed along the (111) direction whose unit cell repeats in every three planes $(A B C A B C)$. For two layers of $P t$ spacer (Figure4.1), a unit cell of 26 atoms from the $(A B C A B C A B C)$ periodic series is engineered in the form of a tetragonal lattice with a lattice parameter $12.12 \AA$. The other two sides of the unit cell are taken as $15 \AA$ to ensure negligible interaction of the nanowire with its image in the $\mathrm{x}$ and the $\mathrm{y}$ direction. From this $A B C A B C$ periodic series, $A$ layer accommodates $7 F e$ atoms; $B$ and $C$ layers have $3 P t$ atoms each. To achieve the ferromagnetic coupling, the spins of two $A$ layers are kept in parallel configuration with each other. The first principle density functional approach [35] is used with local spin density approximation for exchange and correlation. The optimized structures in the FM and AFM configurations are obtained using the stringent force criterion of $0.01 \mathrm{eV} / \AA$ for individual atom. During the self-consistent calculation the convergence criterion for energy is taken to be $10^{-6} \mathrm{eV}$. For the calculation, the plane wave basis set and ultra-soft pseudo potential (USPP) is used. The inter layer exchange coupling $(J)$ is calculated from the difference in total energy between the FM and AFM configurations as:

$$
J=\frac{E_{d}^{\uparrow \downarrow}-E_{d}^{\uparrow \uparrow}}{n} .
$$

$\mathrm{n}$ is the number of atoms in the unit cell. In a representative nanowire with two $P t$ spacer layers in the unit cell, we have also used the projected augmented wave (PAW) potential [35] to test the validity of the results for $J$ with respect to the choice of the potential. It is found that the use of PAW potential with $(1 \times 1 \times 1) k$ point sampling within the Monkhorst 
pack (MP) scheme for structure optimization gives a $J$ value of $19.6 \mathrm{meV}$ as compared to 15.4 meV from USPP with the same $k$-point sampling of the Brillouin zone(BZ). Though the use of PAW potential yields a higher $J$ value, the sign of the $J$ value, which is of interest to us, remains unchanged. The sensitiveness of the $J$ is also tested by optimizing the structure for two $P t$ spacer nanowire using $(1 \times 1 \times 3),(1 \times 1 \times 5)$ and $(1 \times 1 \times 7) k$-point samplings of the Brillouin zone within the MP scheme. Use of (1 x 1 x 3 ) $k$-point sampling of the BZ during structural optimization gives the $J$ value of $9.6 \mathrm{meV}$, which changes to 9.5 meV and $9.3 \mathrm{meV}$ for $1 \times 1 \times 5$ and $1 \times 1 \times 7$ k-point samplings respectively. The relative difference in $J$ value between $1 \times 1 \times 5$ and $1 \times 1 \times 7$ is only $2 \%$. It is also important to note that the sign of the $J$ value remains unchanged with respect to the choice of $k$-point sampling. To check the perceptivity of $J$ with respect to number of atoms in the unit cell, the 2 spacer nanowire is also tested in a bigger unit cell with fifty two atoms. It is found that, irrespective of number of atoms in the unit cell the sign of the $J$ remains unchanged. Considering the spin-polarized nature of the problem and relatively larger size of the unit cell, and the excellent convergence in $J$ value in two $P t$ spacer nanowire, we have used $1 \times 1 \times 5 k$-point sampling of the BZ during geometry optimization for the five and eight spacer layer nanowires. For five spacer layers (Figure. 4.1), the 1st and the 3rd $A$ layers are chosen to be the magnetic $F e$ layers whereas the $B C A B C$ layers in between, are chosen as the nonmagnetic $P t$ layers. In the case of eight spacer layers (Figure. 4.1), the 1st and the 10th $A$ layers are the magnetic $F e$ layers whereas the $B C A B C A B C$ layers in between are the nonmagnetic $P t$ layers. The spins in the magnetic layers are aligned in parallel 
and anti-parallel configurations to obtain the FM and AFM coupling between the magnetic layers. Similar procedures as discussed above for two spacer layers are used to obtain the IEC as a function of the spacer length. The $1 \times 1 \times 5 k$-point mesh was used for calculating the electronic band structure and magnetic moments. A large plane wave cut-off of 237.6 $\mathrm{eV}$ is taken to include reasonably large number of plane waves in the basis set and kept fixed for all subsequent calculations. To calculate the local magnetic moment of individual atom, the Wigner-Seitz radii for $F e$ and $P t$ are taken as 2.46 and 2.75 a.u. respectively.

\subsection{Results and discussion}

The results are organized as follows: First stability and magnetic properties are discussed in Sec.4.3.1., following which the variation of IEC with $P t$ spacer is discussed in Sec.4.3.2 while Sec 4.3.3 describes the spin polarized band structures. In Sec 4.3.4 the calculation of conductance at different spacer width is described.

\subsubsection{Stability and magnetic properties}

In case of two spacer layers system, only a minor relaxation from the $A B C A B C$ packing of the $f c c$ structure is noted. The structures are optimized for both FM and AFM configurations. The optimized nanowire structures for different spacer lengths are depicted in 
Figure.4.1

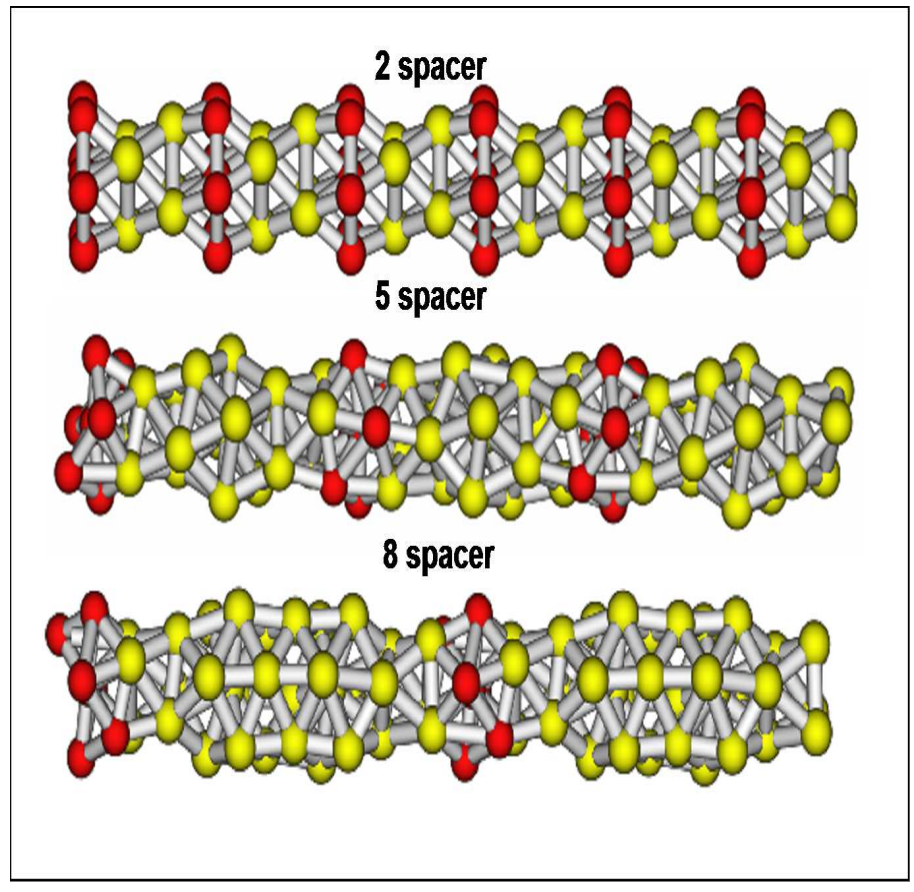

Figure 4.1: Optimized structures for the Fe/Pt nanowire with two Pt spacer, five Pt spacer, and eight Pt spacer layers respectively. Notations: dark gray (red), Fe; light gray (yellow), Pt. Reprinted figure with permission from, Puspamitra.Panigrahi, Ranjit.Pati, Phys. Rev. B. 79, 014411(2009), (c)(2009) The American Physical society.

In the AFM configuration, the atomic level structural relaxations due to spin flips are explicitly included. Any increase in the Pt spacer width can obviously mark a significant distortion from the $A B C A B C$ packing of the fcc due to a strong buckling in the $A$ plane. A similar structural configuration is obtained for the AFM configuration. An increase in $\mathrm{Fe}$ magnetic moment at the $\mathrm{Fe} / \mathrm{Pt}$ interfacial site compared to the $\mathrm{Fe}$ atom at the interface is evident from the analysis of individual magnetic moments in the FM and AFM configurations. Also it is important to note that the magnetic moment of $F e$, far away from the 
interface is almost same as that obtained in the pristine $F e$-nanowire. This trend is also noticed in our first part of the project [67] where a similar increase in magnetic moment at the interfacial $\mathrm{Fe}$ atoms is observed. Furthermore, in $\mathrm{Fe} / \mathrm{Pt}$ bulk structure [62], magnetic moment enhancement for the interfacial $F e$ atom is also reported. To understand the cause of this increase in magnetic moments of $\mathrm{Fe}$ atom at the interface, the magnetic moments of the individual $F e$ atoms at the most affected inter-facial $A$ layer from the $A B C A B C$ series are summarized in Table 4.1

Table 4.1: Comparison of individual magnetic moment (unit $\mu_{B}$ of $\mathrm{Fe}$ atom in 2spacer,5-spacer,8-spacer layer multilayered nanowire systems in both FM and AFM configuration with that of corresponding $\mathrm{Fe}$ atoms in the pristine $\mathrm{Fe}$ nanowires

\begin{tabular}{|c|c|c|c|c|c|c|c|}
\hline Atom & \multirow{2}{*}{ Pristine } & \multicolumn{2}{|c|}{ 2-spacer-layer } & \multicolumn{2}{c|}{ 5-spacer-layer } & \multicolumn{2}{c|}{ 8-spacer-layer } \\
\cline { 3 - 8 } & & ferro & anti-ferro & ferro & anti-ferro & ferro & anti-ferro \\
\hline Fe1 & 0.990 & 2.257 & 1.877 & 2.231 & 2.137 & 2.197 & 2.196 \\
Fe2 & 2.746 & 3.085 & 2.911 & 3.175 & 3.165 & 3.088 & 3.088 \\
Fe3 & 2.743 & 3.085 & 2.911 & 2.997 & 3.090 & 3.088 & 3.089 \\
Fe4 & 2.746 & 3.107 & 3.037 & 3.054 & 3.067 & 3.087 & 3.089 \\
Fe5 & 2.743 & 3.107 & 3.037 & 2.725 & 3.074 & 3.065 & 3.064 \\
Fe6 & 2.743 & 3.108 & 3.037 & 2.725 & 3.056 & 3.065 & 3.063 \\
Fe7 & 2.745 & 3.108 & 3.037 & 2.720 & 3.012 & 3.087 & 3.088 \\
\hline
\end{tabular}

A substantial change in magnetic moment for the core $F e$ atom is noted between pristine and multilayered nanowire (Table 4.1). The increase in magnetic moment can be explained by the spin flip and multi-step electron transferprocess in the similar way as explained in Chapter 1. 


\subsubsection{Switching of IEC with Platinum spacer}

The Figure. 4.2 depicts the calculated IEC as a function of spacer layer thickness. It shows that the magnitude of $J$ value decreases with the increase in spacer width. Both $1 \times 1 \times 1$ and $1 \times 1 \times 5 k$-point sampling of the $B Z$ yield similar trend in IEC.

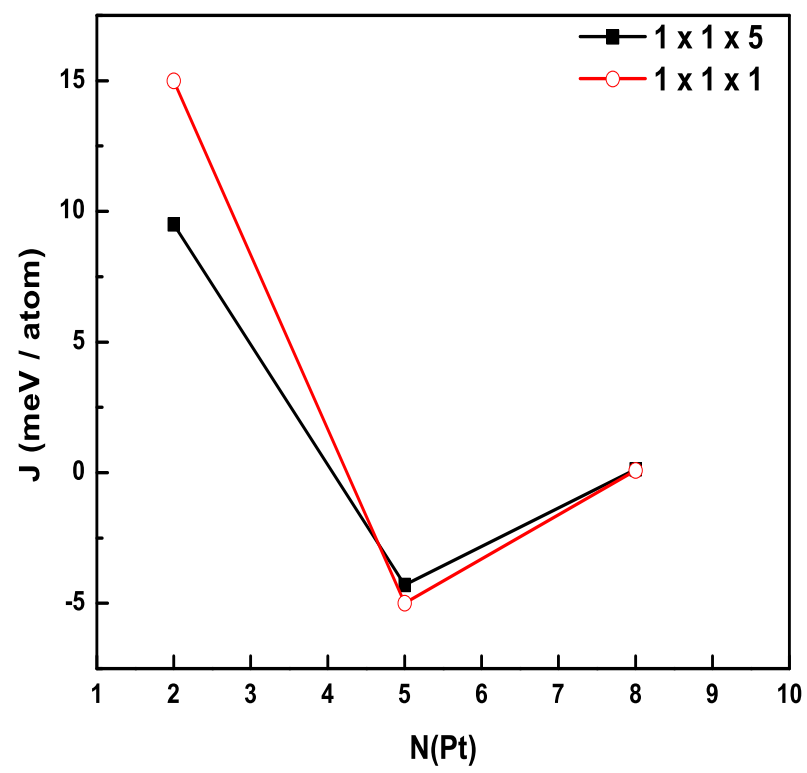

Figure 4.2: Calculated inter layer exchange coupling ( $J$ ), as a function of number of non-magnetic Pt spacer layers $N(P t)$ in the nanowire structures. $1 \times 1 \times 1$ and $1 \times 1 \times 5$ represent the k-point sampling of the BZ used to obtain the respective results. Reprinted figure with permission from, Puspamitra.Panigrahi, Ranjit.Pati, Phys. Rev. B. 79, 014411(2009), (C)(2009) The American Physical society.

Again the $J$ value is found to be substantially smaller for the eight $P t$ spacer layer in the unit cell. However, the most exciting aspect in this Figure. 4.2 is the switching in the sign of $J$. Increasing the number of Pt spacer layers from two to five bring about a sign change in the $J$ value i.e. becoming negative. The AFM configuration is stable for two spacer layer 
system. Where as the FM configuration is found to be more stable for five spacer layer. Further, increasing the number of spacer layer from five to eight again lead to switching in the sign of $J$ value suggesting the stability of the AFM configuration over the FM ordering. In bulk multilayered system, similar outcome in $J$ value switching takes place with the increase in spacer width [5, 6, 7], has been elucidated invoking RKKY and QW model. It is worth to mention that, very recently, Bruno and colleagues [76] have demonstrated a similar oscillation in the exchange coupling between two magnetic adatoms by varying the size of the atomic spacer chain. The magnetic moment per atom were analyzed layer wise to explore the concept of switching in $J$ value in the nanowires. The results are summarized in Figure. 4.3

It is evident from this figure that for two $P t$ spacer system, because of the strong overlap between the inter layer $P t$ wave functions, Pauli's exclusion principle requires the spins in $P t$ layer to be in anti parallel alignment. Thus, the negative direct exchange interaction is favored over the positive direct exchange interaction (parallel spin alignment) between $P t$ layers. These magnetic arrangements in $P t$ layers favor indirect RKKY type exchange interaction between the $\mathrm{Fe}$ layers resulting in a stable AFM coupling. Whereas, as the distance between spin polarized $P t$ layers (2 and 5;2 and 6;3 and 5;3 and 6) increases in case of $5 P t$ spacer system (Figure. 4.4), the positive direct exchange interaction is favored over the negative direct exchange.

Thus, the FM coupling between $F e$ layers is favored over the AFM coupling. The mag- 


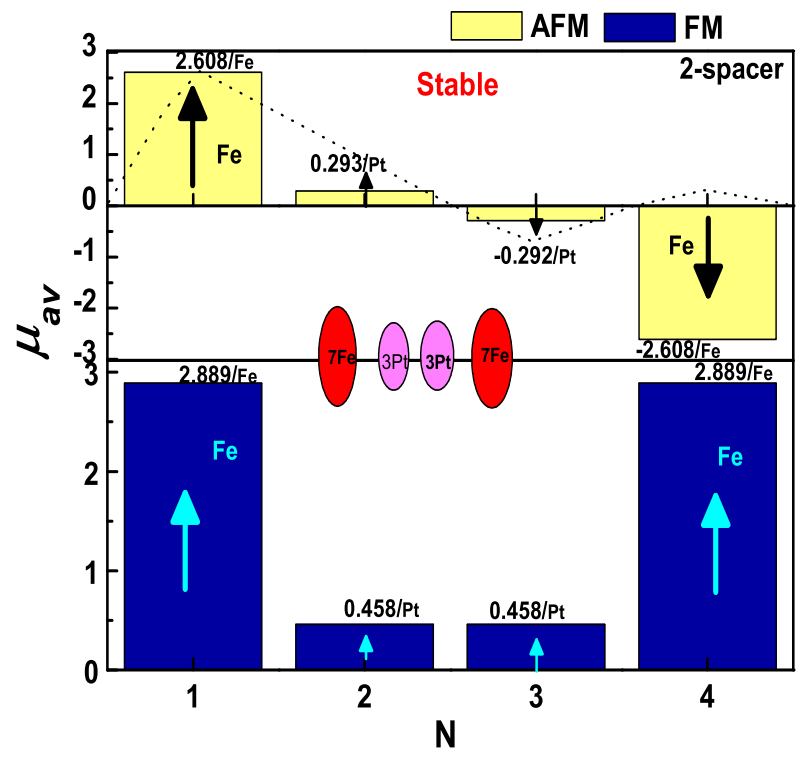

Figure 4.3: Histogram plot for the average magnetic moment per atom $\left(\mu_{a v}\right)$ in ferromagnetic (FM) and anti-ferromagnetic (AFM) configurations for two Pt spacer. Reprinted figure with permission from, Puspamitra.Panigrahi, Ranjit.Pati, Phys. Rev. B. 79, 014411(2009), (C)(2009) The American Physical society

nitude of magnetic moment per atom in the fourth $P t$ layer is substantially smaller (Figure. (4.4) to initiate the negative direct exchange interaction between the $P t$ atoms within the layers as seen for two $P t$ spacer. Indirect RKKY exchange interaction of a different period can also give explanation for the stability of the FM coupling in the case of five layer $P t$ spacer. It is also noteworthy to point out that the small asymmetry in the magnitude of average magnetic moment between the $\mathrm{Fe}$-A layers, shown in Figure. 4.4 is due to small local structural asymmetry around the $A$ layer in the optimized structure. Going in the ascending order with eight $P t$ spacer layers in the unit cell of the wire, with the increase of distance between $\mathrm{Fe}$ layers, the super exchange interaction plays the dominant role in 


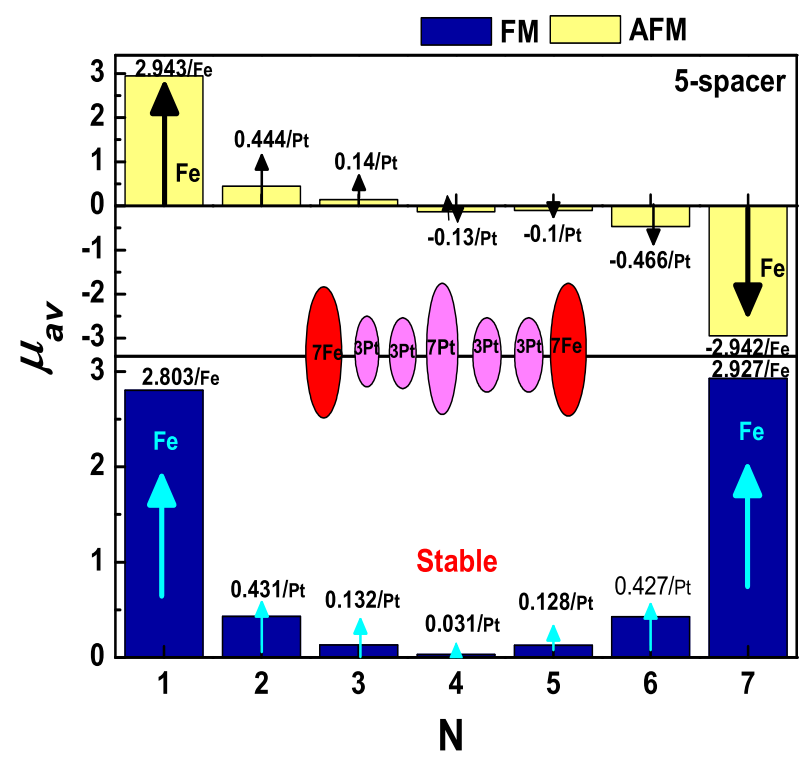

Figure 4.4: Histogram plot for the average magnetic moment per atom $\left(\mu_{a v}\right)$ in ferromagnetic (FM) and anti-ferromagnetic (AFM) configurations for five Pt spacer. Reprinted figure with permission from, Puspamitra.Panigrahi, Ranjit.Pati, Phys. Rev. B. 79, 014411(2009), (C)(2009) The American Physical society.

favoring the AFM coupling over the FM coupling between the $F e$ layers. The layers 4, 5, 6, and 7, as shown in Figure. 4.5 have almost zero magnetic moment per atom. These $P t$ atoms are covalently bonded resulting in the stability of the AFM coupling mediated by these non-magnetic $P t$ spacer atoms.

\subsubsection{Spin polarized energy bands}

To conceptualize an atomic level phenomenon of the switching of $J$ and its role in the electronic properties of the nanowire, the spin polarized energy bands are calculated for the 


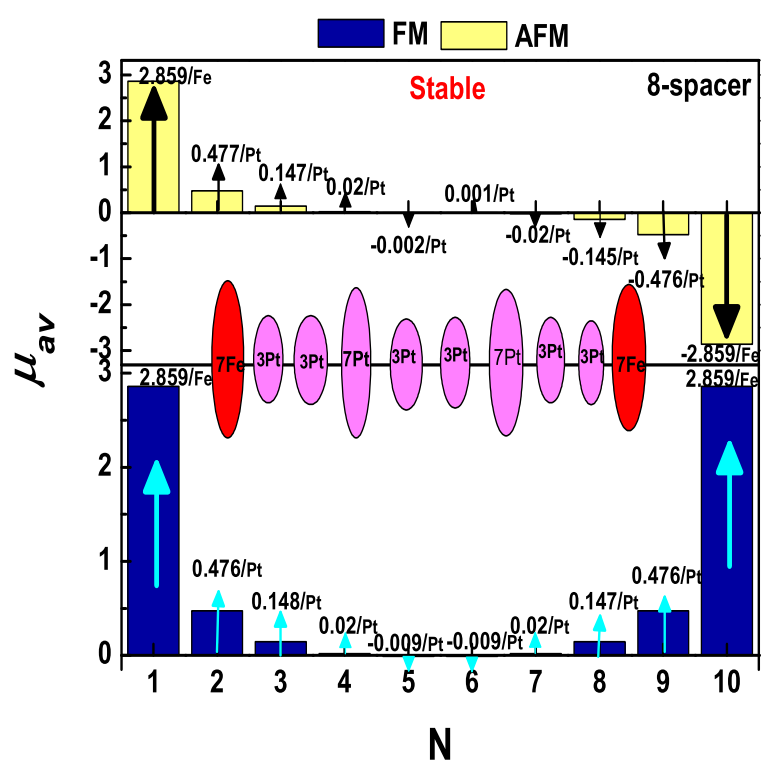

Figure 4.5: Histogram plot for the average magnetic moment per atom $\left(\mu_{a v}\right)$ in ferromagnetic (FM) and anti-ferromagnetic (AFM) configurations for eight Pt spacer. Reprinted figure with permission from, Puspamitra.Panigrahi, Ranjit.Pati, Phys. Rev. B. 79, 014411(2009), (C)(2009) The American Physical society.

three representative nanowires. The results obtained using $1 \times 1 \times 5 k$-point sampling of the BZ are summarized in Figure 4.6 .

In the case of two spacer layers in the unit cell, for the FM configuration (Figure. 4.7), the $\alpha$ valence band and conduction band near the Fermi energy illustrate a clear $F e(s, p, d)$ with $\operatorname{Pt}(s, p, d)$ hybridization; the $\beta$ valence band is mostly $F e(d)$ and $P t(d)$ hybrid band, and $\beta$ conduction band illustrates a dominant $F e(d)$ character. Wherever there is a stable AFM configuration, both the valence band and conduction band near the Fermi energy are mostly $F e(d, p)$ and $P t(d, p)$ hybrid bands. Thus the strong $d-p$ hybridization favors the AFM coupling over the FM coupling in the case of two Pt spacer layers in the unit cell. 


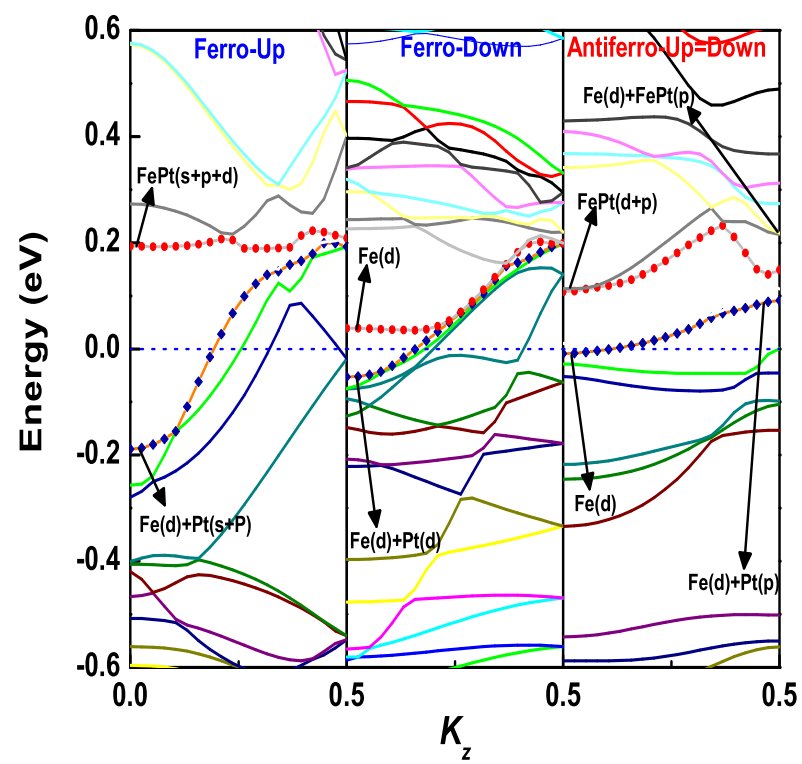

Figure 4.6: Spin polarized energy band structures for 2-spacer system.The Fermi energy is set at $E=0$. Notations: gray (red) circle, conduction band; gray (blue) diamond, valence band.Reprinted figure with permission from, Puspamitra.Panigrahi, Ranjit.Pati, Phys. Rev. B. 79, 014411(2009), (C)(2009) The American Physical society.

Similar $d-p$ hybridization favoring the AFM configuration over the FM configuration was reported in NiAl nanowire [64].

For the five spacer layer nanowire in the FM (stable) configuration, both spin up valence and conduction bands near the Fermi energy (Figure. 4.7) are found to have $P t(d)$ character.

But as we move away from the high symmetry point the bands develop $F e / P t(d, p)$ hybrid character contributing to the stability of the FM configuration. In the spin down case, both valence and conduction bands are $F e(d)$ and $P t(d)$ hybrid bands with dominant $F e(d)$ character at the high symmetry point. In the case of the AFM configuration both valence 


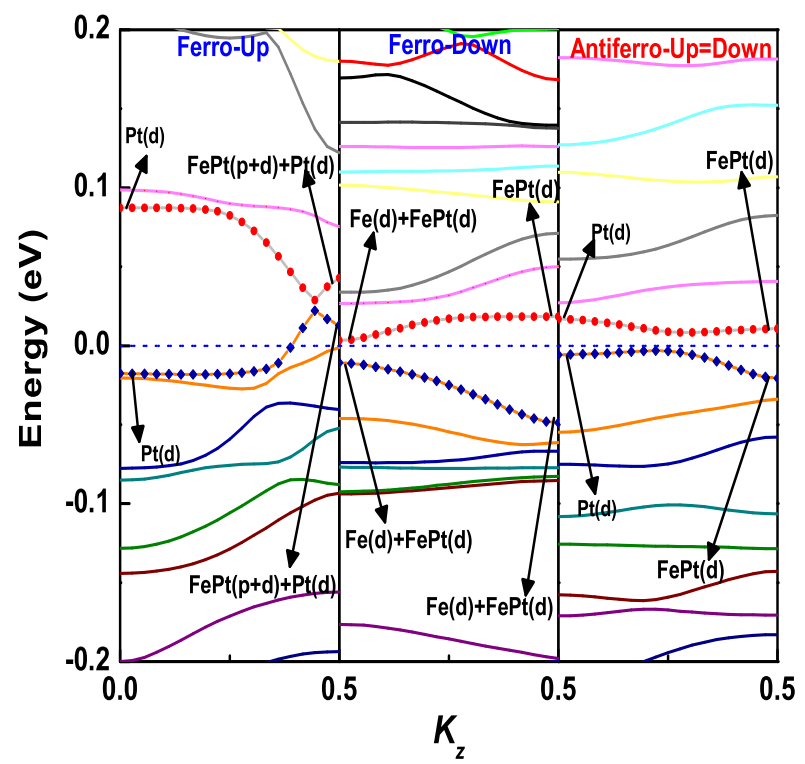

Figure 4.7: Spin polarized energy band structures for 5-spacer system. The Fermi energy is set at $E=0$. Notations: gray (red) circle, conduction band; gray (blue) diamond, valence band. Reprinted figure with permission from, Puspamitra.Panigrahi, Ranjit.Pati, Phys. Rev. B. 79, 014411(2009), (C)(2009) The American Physical society.

and conduction bands are primarily $P t(d)$ bands. Thus, the weakening of the $F e-P t$ hybridization in the AFM configuration case is resulting in an unstable AFM ordering. In the case of eight $P t$ spacer layers in the unit cell, the valence and conduction bands near the Fermi energy are mostly $P t(d)$ bands (Figure. 4.8) with very little $F e(d)$ character. Here bonding between $P t$ atoms is mostly covalent in nature.

Thus the strong covalency within the $P t$ layer favors the AFM coupling between $\mathrm{Fe}$ via the super exchange interaction. 


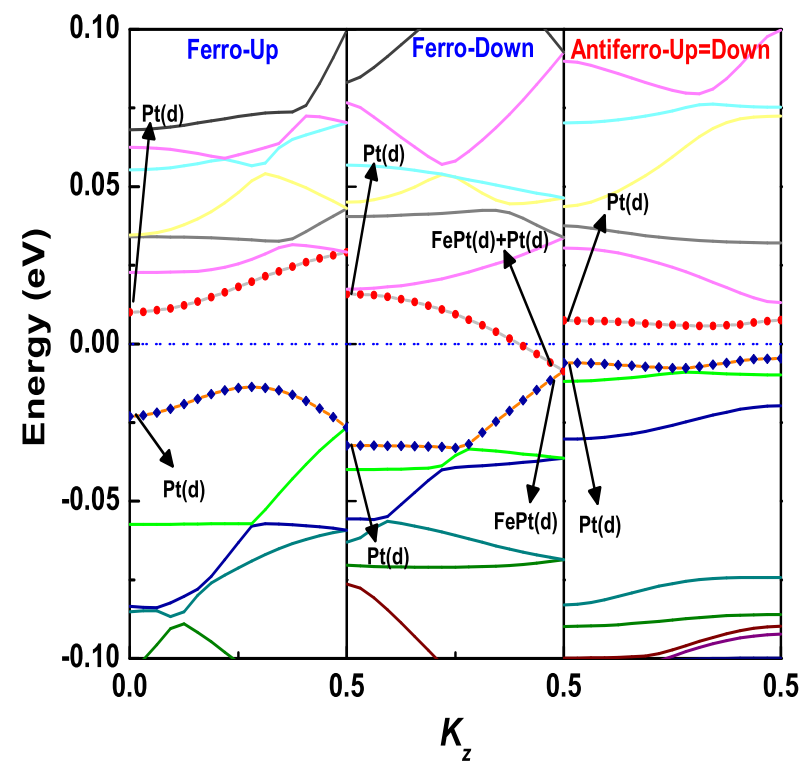

Figure 4.8: Spin polarized energy band structures for 8-spacer system.The Fermi energy is set at $E=0$. Notations: gray (red) circle, conduction band; gray (blue) diamond, valence band. Reprinted figure with permission from, Puspamitra.Panigrahi, Ranjit.Pati, Phys. Rev. B. 79, 014411(2009), (C)(2009) The American Physical society.

\subsubsection{Conductance}

To further understand the $J$ switching and its implication on magneto resistance for practical applications, we have calculated the polarization, conductance and magneto resistance for different spacer length. These results obtained using $1 \times 1 \times 5 k$-point sampling of the BZ are summarized in Table 4.2 From Table 4.2, one can notice switching in the sign of the polarization at the Fermi energy between two and five spacer system. Increasing from five to eight spacer layers in the unit cell, the polarization at the Fermi energy again changes sign. This polarization switching is expected to affect the magneto conductance of the wire. 
Table 4.2: Calculated Polarization, Conductance, and GMR in the nanowire for different spacer lengths.

\begin{tabular}{|c|c|c|c|c|c|c|}
\hline \multirow{2}{*}{ Parameters } & \multicolumn{2}{|c|}{ 2-spacer } & \multicolumn{2}{c|}{ 5-spacer } & \multicolumn{2}{c|}{ 8-spacer } \\
\cline { 2 - 7 } & ferro & anti-ferro & ferro & anti-ferro & ferro & anti-ferro \\
\hline Polarization(p) & -0.9192 & 0 & 0.3385 & 0 & -0.3 & 0 \\
Conductance(G) & 1.2713 & 0.544 & 0.898 & 1.125 & 0.7848 & 0.58 \\
\hline GMR & \multicolumn{2}{|c|}{$57 \%$} & \multicolumn{2}{c|}{$-27 \%$} & \multicolumn{2}{c|}{$26 \%$} \\
\hline
\end{tabular}

The Polarization(p) is defined as

$$
p=\frac{\operatorname{Dos}_{E_{f}}(\alpha)-\operatorname{Dos}_{E_{f}}(\beta)}{\operatorname{Dos}_{E_{f}}(\alpha)+\operatorname{Dos}_{E_{f}}(\beta)}
$$

The $\operatorname{Dos}_{E_{f}}(\alpha)$ corresponds to the $\operatorname{spin} \operatorname{up}(\alpha)$ density of states at the Fermi energy and $\operatorname{Dos}_{E_{f}}(\beta)$ corresponds to spin down $(\beta)$ density of states at the Fermi energy. Using Jullier's model [77], we have calculated the conductances. The Conductance $(\mathrm{G})$ in the FM case is:

$$
G_{F M}=\left[\operatorname{Dos}_{E_{f}}(\alpha)\right]^{2}+\left[\operatorname{Dos}_{E_{f}}(\beta)\right]^{2}
$$

In the case of AFM, the conductance is given by:

$$
G_{A F M}=2 \operatorname{Dos}_{E_{f}}(\alpha)^{2} \operatorname{Dos}_{E_{f}}(\beta)=2\left[\operatorname{Dos}_{E_{f}}(\alpha)\right]^{2},
$$

The GMR value is calculated from the conductance $G_{F M}$ and $G_{A F M}$ as:

$$
G M R=\frac{G_{F M}-G_{A F M}}{G_{F M}} \times 100 \%
$$


The GMR value obtained for different spacer configurations also shows switching in sign as noted for $J$.

\subsection{Summary}

In summary, using first principles density functional approach, we report a comprehensive study on the role of atomic scale structural heterogeneity at the magnetic/non-magnetic interface in modulating the IEC in $1 \mathrm{D} \mathrm{Fe} / \mathrm{Pt}$ nanowire. We found enhancement in the magnetic moment of the $\mathrm{Fe}$ at the $\mathrm{Fe} / \mathrm{Pt}$ interface as compared to the magnetic moment of the $\mathrm{Fe}$ atom away from the interface. A mechanism based on multi-step electron transfer and spin-flip is proposed to explain the increased magnetic moment of the interfacial $\mathrm{Fe}$ atom. The $J$ value as well as GMR is found to switch sign as the spacer width in the nanowire increases. Magnitude of $J$ value is found to decrease substantially for larger spacer width. The competition among short range and long range direct exchange, indirect

exchange, and super exchange depending upon the spacer width is found to be responsible for the non-monotonous sign in $J$. 


\section{Chapter 5}

\section{Role of spacer on Interlayer Exchange}

\section{coupling in multilayered nanowire}

\subsection{Introduction}

Deciphering the interlayer exchange coupling (IEC) and other functional characteristics in $\mathrm{Fe} / \mathrm{Pt}$ multilayered nanowire system in our previous studies, our inquisitiveness drive us to look into the other nanowire systems to further our understanding. In this part of this project, the $\mathrm{Fe} / \mathrm{Pd}, \mathrm{Fe} / \mathrm{Cu}, \mathrm{Fe} / \mathrm{Ag}, \mathrm{Fe} / \mathrm{Au}$ nanowire systems were analyzed with respective spacer layer of Palladium (Pd), Copper $(\mathrm{Cu})$, Silver $(\mathrm{Ag})$, and Gold $(\mathrm{Au})$ by varying their thicknesses. The oscillatory behavior of IEC with regard to various systems includ- 
ing all these combinations were studied in depth in bulk heterostructures. In addition, the IEC in different magnetic/non-magnetic bulk heterostructures eg. $(\mathrm{Co} / \mathrm{Pt}, \mathrm{Co} / \mathrm{Cu}, \mathrm{Co} / \mathrm{Cr}$, $\mathrm{Co} / \mathrm{Ru}, \mathrm{Fe} / \mathrm{Cr}$ ), displaying damped oscillatory behavior were also reported [5, 4, 6]. The RKKY model and the QW model were the basis for explaining the theoretical aspect of the damped oscillatory behavior of these systems. For $f c c C o / C u$ bulk structure, the density of states and magnetic coupling as well as the giant magnetoresistance found to have almost same period of oscillation. The oscillatory magnetic coupling of these systems are explained on the basis of QW model by calculating the total energy of all occupied QW states and observing its periodic jump with increase in spacer thickness as and when a new state crosses the Fermi level. Subsequently, detailed theoretical predictions have also been made for long and short period oscillation of IEC along certain crystallographic direction, for the $\mathrm{Fe} / \mathrm{Au} / \mathrm{Fe}, \mathrm{Fe} / \mathrm{Cu} / \mathrm{Fe}$ and $\mathrm{Co} / \mathrm{Cu}$ sandwiches [78, 79, 80]. To realize the universality of damped oscillatory behavior of IEC with regard to other qualified spacer systems mentioned above, we have extended our study further into multilayered nanowire structure involving $P d, C u, A g$ and $A u$ as spacer. This will give insight into the functionality of these multilayered nanowire systems in comparison to $\mathrm{Fe} / \mathrm{Pt}$ nanowires studied in depth in the previous Chapter. The underlying mechanism involving the sign and magnitude of exchange coupling energy, $J$, as a function of nature and dimension of spacer which are of interest are unraveled with regard to these unexplored nanowire systems. The exchange coupling energy and its switching pattern between the ferromagnetic layers with respective spacers like $P d, C u, A g, A u$ with varying thicknesses were discussed, which further 
our perception about these multilayered nanowire systems. This Chapter is organized with the following Sections. Section 5.2 briefly describes the theoretical approach; Section 5.3 discusses the results and discussion; Section 5.4 summarizes our main conclusions.

\subsection{Theoretical Approach}

In the line with the structure of the $\mathrm{Fe} / \mathrm{Pt}$ nanowire, the atomic arrangement in studied $\mathrm{Fe} / \mathrm{Pd}, \mathrm{Fe} / \mathrm{Cu}, \mathrm{Fe} / \mathrm{Ag}, \mathrm{Fe} / \mathrm{Au}$ multilayered nanowires are engineered along the $\left(\begin{array}{lll}1 & 1 & 1\end{array}\right)$ direction with units cell repeating in every three planes $(A B C A B C)$. Here, we have emphasized on the nature of the non-magnetic spacers sandwiched in between ferromagnetic $\mathrm{Fe}$ layers. For this purpose, we have considered two and five layers of non-magnetic spacers in all the four representative nanowire systems. In case of two spacer systems, the units cell consists of 26 atoms from the $(A B C A B C A B C)$ periodic series. The tetragonal unit cell of each representative nanowire has lattice parameter $12.12 \AA$ the other two sides of the unit cells are $15 \AA$, ensuring negligible interaction of the nanowire with its images along other two axes. The geometrical structure optimization for both FM and AFM coupling between the $F e$ layers with specific spacer layer of choice is performed. Both the criteria for the force on individual atom and energy convergence are same as that of the $\mathrm{Fe} / \mathrm{Pt}$ system discussed earlier to have a broad comparison between the systems. The exchange coupling energy, $J$, the energy difference between FM and AFM configurations is also calculated in the similar fashion. As discussed in the previous chapter, the sign of $J$ value remains un- 
changed with respect to the choice of $k$ point sampling of the BZ. We have taken $1 \times 1 \times 5$ $k$-point sampling for the geometrical structure optimization of all representative nanowires. The same $1 \times 1 \times 5 k$-point sampling of the $\mathrm{BZ}$ is considered for electronic band structure and magnetic moment calculations. For calculation of local magnetic moment of individual atom, the Wigner Seitz radii for $F e, P d, C u, A g, A u$ are taken as 2.46, 2.71, 2.48, 2.84, 2.84 a.u respectively. The ultra-soft pseudo potential (USPP) is used for all the calculations.

\subsection{Results and Discussion}

The results are presented as follows. Section 5.3.1 discusses structure, stability, and magnetic properties of nanowires; Sec 5.3.2 discusses with variation of $\mathrm{J}$ with spacer length along with the spin polarized band structuresof the multilayered nanowire.

\subsubsection{Structure stability and magnetic properties}

For $\mathrm{Fe} / \mathrm{Pd}, \mathrm{Fe} / \mathrm{Cu}, \mathrm{Fe} / \mathrm{Au}, \mathrm{Fe} / \mathrm{Ag}$ systems, the optimized nanowire structure with 2-spacer and 5 spacer length are presented in Figure. 5.1 For the AFM configurations, the atomic level structural relaxation due to spin flip is explicitly considered. Similar structural configurations is observed for both the FM and AFM configurations irrespective of spacer length and nature. In 5-spacer system, the increase in concentration of $P d, C u, A g, A u$ leads to 
significant structural distortion due to lattice mismatch. similar structural features were noted in previously studied $\mathrm{Fe} / \mathrm{Pt}$ system.

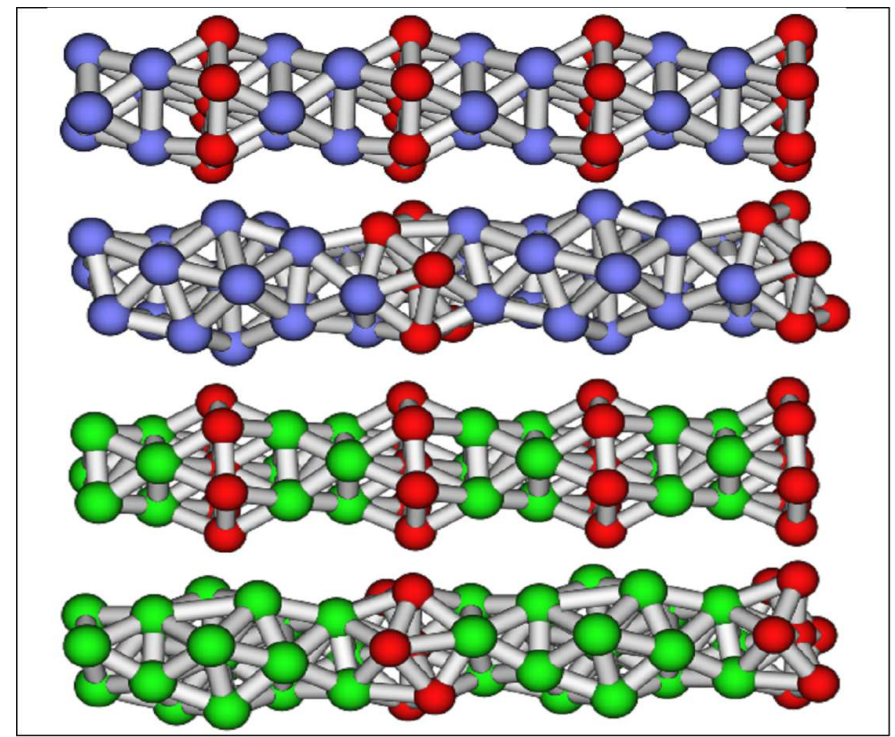

Figure 5.1: Optimized structures for the Fe/spacer nanowire with two spacer, and five spacer layers respectively in the FM and AFM configuration. The top two wires correspond to the structure in FM configuration; the bottom two figures correspond to the structure in AFM configuration. Notations: dark gray (red), Fe; light gray (green and blue), spacers. Spacer atoms: $\mathrm{Pd}, \mathrm{Cu}, \mathrm{Au}, \mathrm{Ag}$.

For the $\mathrm{Fe} / \mathrm{Pd}$ nanowire, the stability is found to increases when compared to that of the pristine $\mathrm{Fe}$ nanowire. A similar trend was observed in the case of $\mathrm{Fe} / \mathrm{Pt}$ nanowire system. For $\mathrm{Fe} / \mathrm{Cu}, \mathrm{Fe} / \mathrm{Ag}, \mathrm{Fe} / \mathrm{Au}$ systems, the stability shows rather a decreasing trend with the increase of spacer size. This can be explained by comparing the bulk cohesive energy of the spacers with that of the bulk Iron. For $C u, A g$ and $A u$, the bulk cohesive energies are lower compared to that of the Iron. Thus, these spacer rich nanowires are less stable than the pristine $F e$ nanowire. In $F e / P d$ nanowire, the increase in stability is due to strong $F e-P d$ hybridization. The magnetic properties of these representative nanowires are also 
assessed, and compared to that with the magnetic properties of the pristine $\mathrm{Fe}$ nanowire system. A significant increase in magnetic moment of the $F e$ atom at the interfacial layer is observed as found in $\mathrm{Fe} / \mathrm{Pt}$ multilayered nanowire. This increase in magnetic moment can be explained by the proposed spin flip and multi-step electron transfer process explained in Chapter 1.

\subsubsection{The role of spacers on IEC}

The cohesive energy, $E_{c}$, of the $\mathrm{Fe} / \mathrm{Pd}, \mathrm{Fe} / \mathrm{Cu}, \mathrm{Fe} / \mathrm{Ag}$, and $\mathrm{Fe} / \mathrm{Au}$ nanowires are calculated in the similar fashion as described in Chapter 3. The exchange coupling energy, $J$, the difference in energy between FM and AFM is also calculated as mentioned in Chapter 4. To understand the behavior of IEC as a function of nature and thickness of spacer width, the $E_{c}$ and $J$ for all four nanowire systems are presented in Table. 5.1 for $(1 \times 1 \times 1) \mathrm{k}-$ point sampling of the Brillouin zone. Table. 5.2 represents results obtained with $(1 \times 1 \times 5)$ k-point sampling of the BZ.

Table 5.1: Results for exchange coupling energy $J$ in 2-spacer, and 5-spacer multilayered nanowire systems obtained with $(1 \times 1 \times 1)$ k-point sampling of the Brillouin zone.

\begin{tabular}{|c|c|c|c|c|c|c|}
\hline \multirow{2}{*}{ spacer } & \multicolumn{3}{|c|}{ 2-spacer-layer } & \multicolumn{3}{c|}{ 5-spacer-layer } \\
\cline { 2 - 7 } & $E_{c(f m)}(\mathrm{eV})$ & $E_{c(a f m)}(\mathrm{eV})$ & $\mathrm{J}(\mathrm{meV})$ & $E_{c(f m)}(\mathrm{eV})$ & $E_{c(a f m)}(\mathrm{eV})$ & $\mathrm{J}(\mathrm{meV})$ \\
\hline $\mathrm{Fe} / \mathrm{Pd}$ & -4.825 & -4.845 & 20.61 & -5.047 & -5.044 & -2.71 \\
$\mathrm{Fe} / \mathrm{Cu}$ & -4.0131 & -4.0011 & -12.00 & -3.8063 & -3.8042 & -2.096 \\
$\mathrm{Fe} / \mathrm{Ag}$ & -3.615 & -3.608 & -6.584 & -3.259 & -3.2592 & -0.307 \\
$\mathrm{Fe} / \mathrm{Au}$ & -4.055 & -4.052 & -3.692 & -3.823 & -3.827 & 4.4038 \\
\hline
\end{tabular}


Table 5.2: Results for exchange coupling energy $J$ in 2-spacer,and 5-spacer multilayered nanowire systems obtained with $\left(\begin{array}{llll}1 & \text { x } & 1 & \text { x }\end{array}\right)$ k-point sampling of the Brillouin zone.

\begin{tabular}{|c|c|c|c|c|c|c|}
\hline \multirow{2}{*}{ spacer } & \multicolumn{3}{|c|}{ 2-spacer-layer } & \multicolumn{3}{c|}{ 5-spacer-layer } \\
\cline { 2 - 7 } & $E_{c(f m)}(\mathrm{eV})$ & $E_{c(a f m)}(\mathrm{eV})$ & $\mathrm{J}(\mathrm{meV})$ & $E_{c(f m)}(\mathrm{eV})$ & $E_{c(a f m)}(\mathrm{eV})$ & $\mathrm{J}(\mathrm{meV})$ \\
\hline $\mathrm{Fe} / \mathrm{Pd}$ & -4.8325 & -4.8414 & 8.880 & -5.0466 & -5.0455 & -1.101 \\
$\mathrm{Fe} / \mathrm{Cu}$ & -3.9857 & -3.9841 & -0.811 & -3.8046 & -3.8036 & -1.005 \\
$\mathrm{Fe} / \mathrm{Ag}$ & -3.6074 & -3.6088 & 1.434 & -3.2655 & - & - \\
$\mathrm{Fe} / \mathrm{Au}$ & -4.0489 & -4.0506 & 1.669 & - & -4.0506 & - \\
\hline
\end{tabular}

Here $E_{c(f m)}$ and $E_{c(a f m)}$ represents the cohesive energy in ferromagnetic and anti-ferromagnetic configurations respectively. To understand the role of non-magnetic spacer in switching behavior of $J$ in different nanowire system, the average magnetic moment $\mu_{a v}$ (per layer) is calculated for all the four. The result for average magnetic moment, $\mu_{a v}$, associated with spacer layers in the representative nanowire systems are presented in Table. 5.3

Table 5.3: Comparison of individual average magnetic moment (unit $\mu_{B}$ associated with soacer layers in 2-spacer multilayered nanowire systems.

\begin{tabular}{|c|c|c|c|c|c|c|c|c|}
\hline 2-spacers & \multicolumn{2}{|c|}{$\mathrm{Fe} / \mathrm{Pd}\left(\mu_{a v}\right)$} & \multicolumn{2}{c|}{$\mathrm{Fe} / \mathrm{Cu}\left(\mu_{a v}\right)$} & \multicolumn{2}{c|}{$\mathrm{Fe} / \mathrm{Ag}\left(\mu_{a v}\right)$} & \multicolumn{2}{c|}{$\mathrm{Fe} / \mathrm{Au}\left(\mu_{a v}\right)$} \\
\cline { 2 - 9 } Layer & $\mathrm{FM}$ & $\mathrm{AFM}$ & $\mathrm{FM}$ & $\mathrm{AFM}$ & $\mathrm{FM}$ & $\mathrm{AFM}$ & $\mathrm{FM}$ & $\mathrm{AFM}$ \\
\hline $1^{\text {st }}$ & 0.303 & 0.266 & -0.021 & -0.006 & 0.123 & 0.161 & 0.124 & -0.018 \\
$2^{\text {nd }}$ & 0.300 & -0.262 & -0.021 & -0.006 & 0.108 & -0.073 & -0.006 & -0.120 \\
\hline
\end{tabular}

Explanation for each of the multilayered nanowire systems is presented in a separate subheading. 


\subsubsection{Fe/Pd Nanowire}

In the Fe/Pd system, the magnitude of exchange coupling energy, $J$, changes inversely with that of the $P d$ spacer width and simultaneously, it switches from a positive value to negative value as the spacer length increases from two to five. For two $P d$ spacer, the $J$ value is $20.61 \mathrm{meV}$, however reduces to $-2.71 \mathrm{meV}$ for five spacer layer (Table. 5.1) for $(1 \mathrm{x} 1 \mathrm{x}$ 1) $k$-point sampling. In the case $(1 \times 1 \times 5) k$-point sampling of the $\mathrm{BZ}$, the value $J$ 's are $8.9 \mathrm{meV}$ and $-1.1 \mathrm{meV}$ for 2-spacer and 5-spacer system respectively. This trend exactly follows that of the $\mathrm{Fe} / \mathrm{Pt}$ system studied before. To understand the switching of $J$ value in the $\mathrm{Fe} / \mathrm{Pd}$ nanowire system, the magnetic moment per atom were analyzed layer wise. As the non-magnetic $P d$ is the key element, the average magnetic moment of $P d$ atoms per layer is presented in Table. 5.3, From the Table. 5.3, in two $P d$-spacer system, the negative direct exchange interaction favors over the positive direct exchange interaction. Thus, the AFM configuration is more stable than the FM configuration. In five Pd-spacer system, the distance between $(1,5),(1,4),(2,4)$, and $(2,5) P d$ layers increases. Thus, the positive direct exchange interaction favors over the negative exchange interaction between the $P d$ layers. This in turn stabilizes the FM configuration over the AFM configuration.

\section{Spin polarized energy bands:}

The spin polarized energy bands are calculated for the $F e / P d$ nanowires using $1 \times 1 \times 5$ $k$-point sampling of the BZ for two and five spacer system and are depicted in Figure. 5.2 
and Figure. 5.3 respectively. In two-spacer system, for the FM configuration (Figure. 5.2) the $\alpha$ valence band and conduction band near the Fermi energy illustrate a clear Fe with Pd (s, p, d) hybridization. Whereas, the $\beta$ valence band is mostly $F e(d)$ bands.

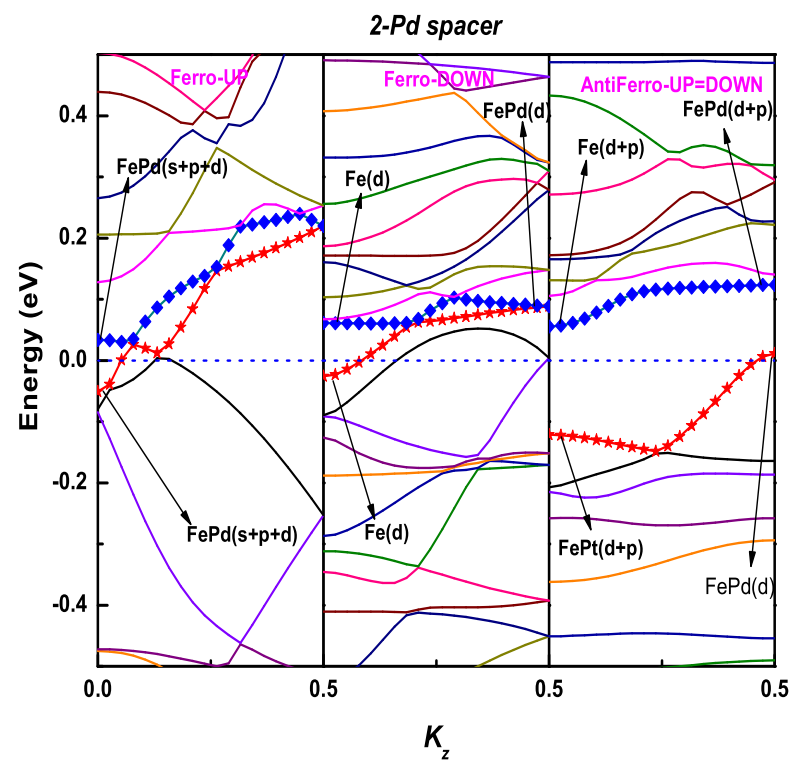

Figure 5.2: Spin polarized energy band structures for 2-spacer Fe/Pd system.The Fermi energy is set at $E=0$. Notations: gray (blue) diamond, conduction band; gray (red) star, valence band.

But, as we move from high symmetry point, the $F e$ and $P d(d)$ hybrid bands dominate. In the stable AFM configuration both the valence and conduction bands are mostly $F e P t(d, p)$ hybrid bands. According to Kramers-Anderson exchange rule, [82, 83] when there is a strong $d-p$ hybridization between magnetic and non-magnetic ions, the AFM coupling between the magnetic ions sitting next to nearest non-magnetic ions favors over the FM coupling. Thus, for 2-spacer systems, the strong $\mathrm{FePd}(d-p)$ hybridization favors the stable AFM coupling. Similar effect has also been observed for $\mathrm{Fe} / \mathrm{Pt}$ and $\mathrm{Ni} / \mathrm{Al}$ multilay- 
ered nanowire system [64, 81]. For both the system, the strong d-p hybridization between magnetic ion and spacer makes the AFM coupling favorable for 2-spacer system. In case of the five-spacer in the unit cell, for FM configuration (Figure. 5.3), the $\alpha$ valence band mostly have $P d(s, p)$ bands and $F e(d)$ characters; the $\alpha$ conduction band is $F e P d(s, p)$ hybrid band. For the $\beta$ valence and conduction bands mostly $F e P d(d)$ character dominates. In case of the AFM configuration, both valence and conduction bands are $F e$ and $P d(d)$ bands.

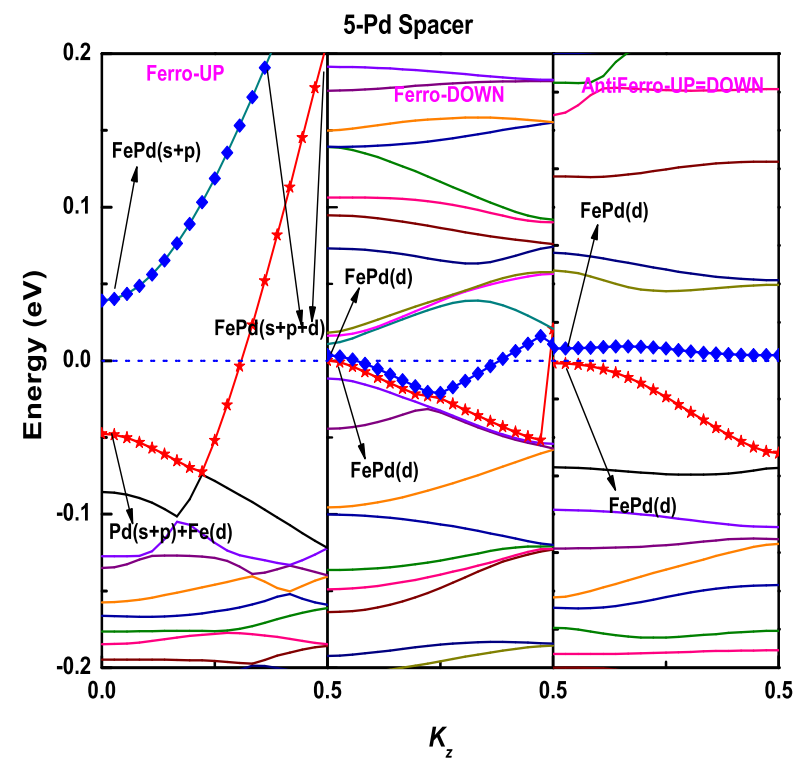

Figure 5.3: Spin polarized energy band structures for 5-spacer Fe/Pd system.The Fermi energy is set at $E=0$. Notations: gray (blue) diamond, conduction band; gray (red) star, valence band.

According to Goodenough-Kanamori [84, 85] rule, superexchange interactions favors antiferromagnetic ordering when the virtual electron transfer is between overlapping orbitals that are each half-filled, but they favor ferromagnetic ordering when the virtual electron 
transfer is from a half-filled to an empty orbital or from a filled to a half-filled orbital. Thus, in the $5 p d$-spacer system, for both the spin up valence and conduction bands in the FM configuration, the strong hybridization between half filled $\left[\mathrm{Fe}\left(3 d^{6}\right]\right.$ and empty $[\mathrm{Pd}$ $\left(5 s^{0}\right)$ ] bands favors the strong FM exchange coupling over the AFM coupling.

\subsubsection{2 $\mathrm{Fe} / \mathrm{Cu}$ Nanowire}

In the $\mathrm{Fe} / \mathrm{Cu}$ system the most striking observation is that unlike the $\mathrm{Fe} / \mathrm{Pt}$ and $\mathrm{Fe} / \mathrm{Pd}$ system, the magnitude of $J$ increases with increase in the $C u$ spacer thickness and it doesn't switch sign. For two and five $\mathrm{Cu}$-spacer systems, the calculated $J$ values are -12.00 and $2.096 \mathrm{meV}$ respectively, obtained using $(1 \times 1 \times 1) k$-point sampling of the BZ (Table. 5.1). For $(1 \times 1 \times 5) k$-point sampling of the $\mathrm{BZ}$, the respective $\mathrm{J}$ values are -0.811 and -1.005 meV (Table. 5.2). As shown in Table. 5.3 in two and five $\mathrm{Cu}$-spacer systems, the direct exchange interaction play a dominant role in stabilizing the FM configuration over the AFM configuration. To understand the role of hybridization we look into the spin polarized band structure in $\mathrm{Fe} / \mathrm{Cu}$ nanowire for both two and five spacer nanowires.

\section{Spin polarized band structure:}

The spin polarized energy bands are calculated for the $\mathrm{Fe} / \mathrm{Cu}$ nanowires using 1 x 1 x 5 $k$-point sampling of the BZ for two and five spacer system. The results are depicted in Figure. 5.4 and Figure. 5.5 respectively. In two-spacer system, for the FM configuration, (Fig. 5.4) the $\alpha$ valence band near the Fermi energy illustrate a clear $\mathrm{FeCu}(s, p)$ hybrid 
band character with some contribution from $F e(d)$.

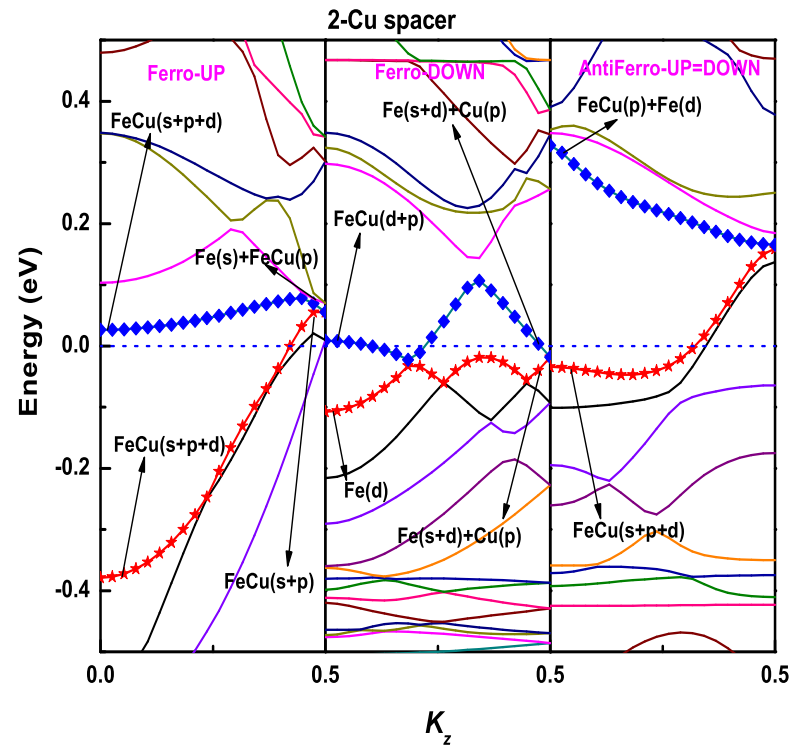

Figure 5.4: Spin polarized energy band structures for 2-spacer $\mathrm{Fe} / \mathrm{Cu}$ system.The Fermi energy is set at $E=0$. Notations: gray (blue) diamond, conduction band; gray (red) star, valence band.

The $\alpha$ conduction band is also mostly $\mathrm{FeCu}(s, p)$ hybrid band with some contribution from $F e(d)$. The $\beta$ valence band is mostly $F e(d)$ band; the conduction band is $F e C u(d, p)$ hybrid band. But, as we move away from the high symmetry point, the $F e(s, d)$ and $C u(p)$ characters contribute to both valence and conduction bands. Here, the same GoodenoughKanamori rule explains the stability of the FM configuration. The hybridization between half filled $\left[\mathrm{Fe}\left(3 d^{6}\right)\right]$ band and filled $\left[\mathrm{Cu}\left(3 p^{6}\right)\right]$ band in spin down case favors strong FM exchange interaction. Also, the half filled $\left[C u\left(4 s^{1}\right)\right]$ hybridizes with completely filled $[F e$ $\left.\left(3 p^{6}\right)\right]$ in spin up case favors the strong FM coupling. In the case of five-spacer in the 
unit cell, for FM configuration, ( Figure. 5.5) the $\alpha$ valence band and conduction band are mostly $\mathrm{FeCu}(s, p)$ hybrid bands. For the spin down case, valence band and conduction band are mostly $\mathrm{Fe}(\mathrm{d})$ bands. But as we move away from the high symmetry points both the valence and conduction bands are found to have $C u(p)$ character.

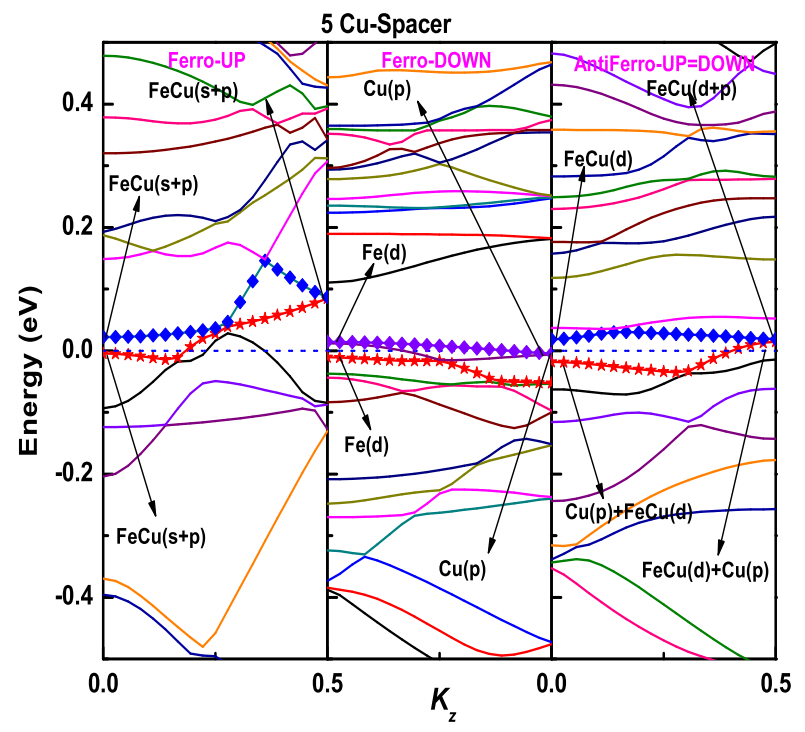

Figure 5.5: Spin polarized energy band structures for 5-spacer Fe/Cu system.The Fermi energy is set at $E=0$. Notations: gray (blue) diamond, conduction band; gray (red) star, valence band.

Thus, the hybridization between half filled $\left[\mathrm{Cu}\left(4 s^{1}\right)\right]$ band and filled $\left[\mathrm{Fe}\left(3 p^{6}\right)\right]$ band in spin down case favors the strong FM exchange interaction. In case of the AFM configuration, the valence band has $C u(p)$ bands and $\mathrm{Fe} / \mathrm{Cu}(\mathrm{d})$ hybrid characters; the conduction band is mostly $\mathrm{FeCu}(d)$ band. Away from the high symmetry point, the $\mathrm{Fe} / \mathrm{Cu}(d, p)$ hybrid characters contributes to both valence and conduction band. 


\subsubsection{Fe/Ag and Fe/Au Multilayered Nanowires}

Unlike the $\mathrm{Fe} / \mathrm{Cu}$ nanowire the trend for two spacer system in $\mathrm{Fe} / \mathrm{Ag}$ and $\mathrm{Fe} / \mathrm{Au}$ nanowires are similar to that of the $\mathrm{Fe} / \mathrm{Pt}$ and $\mathrm{Fe} / \mathrm{Pd}$ systems. Here the calculations are done for both the multilayered nanowires with only 2 layers of nonmagnetic spacers. As used for other nanowires both $(1 \times 1 \times 1)$ k-point and ( $1 \times 1 \times 5)$ k-point grid are used to sample the BZ. From (1 x 1 x 5) k-point sampling results (Table. 5.2); it can be noted that, for both the nanowires with 2-spacer, the AFM coupling favors over the FM coupling. The magnitude of $J$ s for two spacer and five spacer system with (1 x 1 x 1 ) k-point sampling of the $\mathrm{BZ}$ are shown in the table (Table. 5.1). To understand the role of non-magnetic spacer on $J$ value in both the nanowire system, the average magnetic moment per spacer atoms are presented in Table. 5.3. From Table. 5.3, it can be noted that for both $A g$ and $A u$ systems, the negative direct exchange interaction favors over the positive direct exchange interaction. Thus the AFM coupling is more stable than the FM coupling in both $\mathrm{Fe} / \mathrm{Ag}$ and $\mathrm{Fe} / \mathrm{Au}$ multilayered nanowire systems. To identify the role of the hybridization, the spin polarized band structures are studied in both $\mathrm{Fe} / \mathrm{Ag}$ and $\mathrm{Fe} / \mathrm{Au}$ multilayered nanowires with two layers of non-magnetic spacers. Spin polarized band structure:

The calculated spin polarized band structure for the 2-spacer $\mathrm{Fe} / \mathrm{Ag}$ nanowire is presented in Figure 5.6, In the FM configuration, the $\alpha$ valence and conduction bands near the Fermi energy are mostly $\mathrm{FeAg}(s, p)$ hybrid bands. Whereas, the $\beta$ valence band is mostly $\mathrm{FeAg}$ (d) band and the $\beta$ conduction band shows predominantly $F e(d)$ character. In the stable 
AFM configuration, both the valence band and the conduction band are mostly $F e A g(d, p)$ hybrid bands.

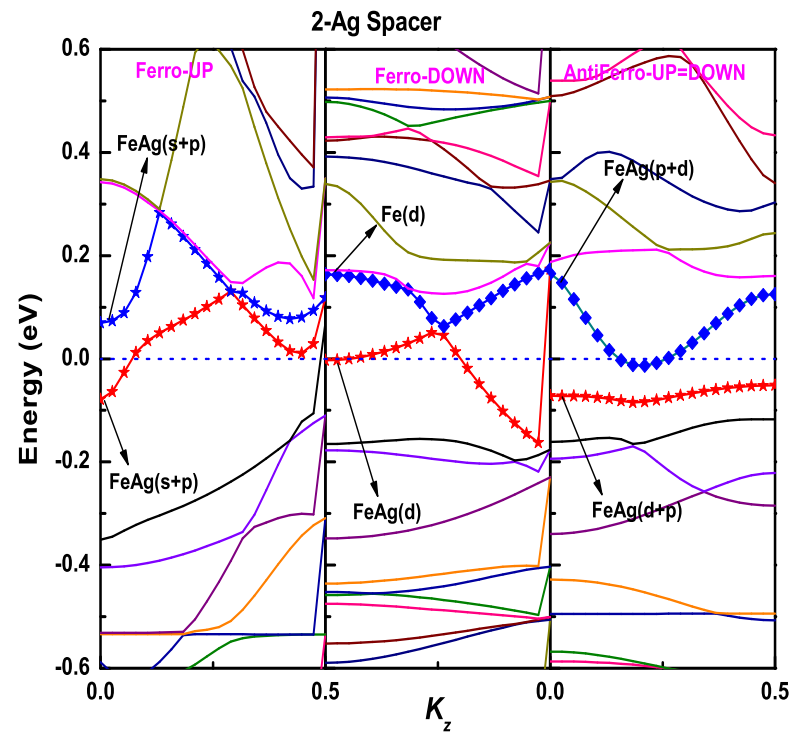

Figure 5.6: Spin polarized energy band structures for 2-spacer Fe/Ag system.The Fermi energy is set at $E=0$. Notations: gray (blue) diamond, conduction band; gray (red) star, valence band.

From the Kramers-Anderson exchange rule, the strong $d-p$ hybrid bands favors the AFM coupling over the FM coupling in 2-spacer $\mathrm{Fe} / \mathrm{Ag}$ multileyered nanowire system. The spin polarized band structure for the 2-spacer $\mathrm{Fe} / \mathrm{Au}$ nanowire is depicted in Figure. 5.7 In the FM configuration, the $\alpha$ valence band near the Fermi energy is mostly $F e A u(s, p, d)$ hybrid band and the conduction band is mostly $F e A u(s, p)$ hybrid band. But near the L-point(away from the high symmetry $\gamma$ point, both valence and conduction band are $F e A u(s, p)$ hybrid bands. The $\beta$ valence band is mostly $F e A u(d)$ hybrid band. The $\beta$ conduction band shows 
predominantly $F e(d)$ character with some contributions from $F e A u(d, p)$ hybrid band. In the stable AFM configuration, both the valence band and conduction band are mostly FeAu $(d, p)$ hybrid bands (Figure. 5.7). Here also, the same Kramers-Anderson exchange rule explains the stability of the AFM configuration over the FM configuration.

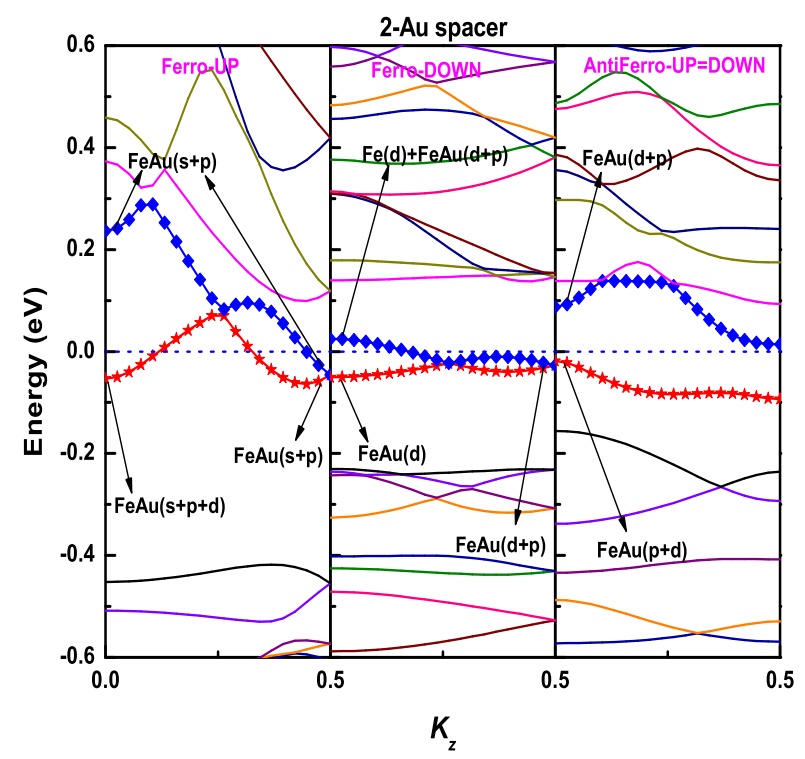

Figure 5.7: Spin polarized energy band structures for 2-spacer Fe/Au system.The Fermi energy is set at $E=0$. Notations: gray (blue) diamond, conduction band; gray (red) star, valence band.

\subsection{Summary}

In this chapter, a wide range of systems are studied and it elucidates the role of different non magnetic spacers in controlling IEC and the behavior of the spin polarized electronic struc- 
ture. Conclusively, the role of atomic scale structural heterogeneity at the magnetic/nonmagnetic interface, in modulating the IEC in $\mathrm{Fe} / \mathrm{Pd}, \mathrm{Fe} / \mathrm{Cu}, \mathrm{Fe} / \mathrm{Ag}$ and $\mathrm{Fe} / \mathrm{Au}$ multilayered nanowire systems, is identified. These systems behave differently depending on the nature and extent of the spacer material used. The mechanism of multi-step electron transfer and spin-flip, proposed in the previous chapter, holds good in explaining the increased magnetic moment of the interfacial magnetic atoms. The $J$ value switches sign with the increase in spacer width in case of $\mathrm{Fe} / \mathrm{Pd}$ system; in case of $\mathrm{Fe} / \mathrm{Cu}$ system it doesn't change sign. The magnitude of $J$ value is found to decrease with the increase in $F e / P d$ nanowire. In case od $\mathrm{Fe} / \mathrm{Cu}$ nanowire an anomalous behavior is noted. Where as $\mathrm{Fe} / \mathrm{Ag}$ and $\mathrm{Fe} / \mathrm{Au}$ nanowires show similar trend as that in $\mathrm{Fe} / \mathrm{Pd}$ and $\mathrm{Fe} / \mathrm{Pt}$ nanowires. These striking similarities and obvious differences between these nanowires and corresponding spacer layers suggest that different strategies need to be adopted for their effective utilization in sprintonic devices. 


\section{Chapter 6}

\section{General Conclusions, Perspectives and}

\section{Future Directions}

\subsection{Conclusions}

The sequence of studies undertaken here deals with the calculation of tunable electronic structure and magnetic properties of multilayered nanowires as a function of size and the nature of the spacer structure. It is evident from the first part of the study that the nonmagnetic spacer layer thicknesses have a role in modulating the magnetic and electronic properties of multilayered nanowire. The stability of nanowires is found to increase by increasing the thickness of the $P t$ layer and consequently, reducing the thickness of the $F e$ 
layer in the $\mathrm{Fe} / \mathrm{Pt} / \mathrm{Fe}$ multilayered nanowire. This in turn, induces a monotonically increasing effect in the average magnetic moment per $F e$ atom quantitatively from $2.49 \mu_{B}$ in a pristine $\mathrm{Fe}_{39}$-nanowire to $2.7 \mu_{B}$ in $\mathrm{Fe}_{13} \mathrm{Pt}_{26}$-nanowire and then to $2.95 \mu_{B}$ for $\mathrm{Fe}_{7} \mathrm{Pt}_{32^{-}}$ nanowire, which is referred as the barcode layer effect. A simple model based on the interface structure is proposed explaining the $\frac{1}{N(F e)}$ dependence trend in $\mu_{a v}$ obtained from the first-principles density functional calculations. A new mechanism based on the spin flip and multi-step electron transfer process is proposed to explain the enhancement of magnetic moments with the increase of non magnetic spacer layer in $\mathrm{Fe} / \mathrm{Pt}$ system. Thus, the role of interfacial bonding in modulation of the magnetic characters is established. The decrease in magnetic moment in a higher diameter nanowire system is also well explained by the quantum confinement effect. Analyzing the spin polarized band structure, a strong dependence of spin-polarized energy bands in the vicinity of the Fermi energy on the nonmagnetic layer thickness was also observed. This suggests the potential applications of these nanowires structure in magneto-electronics or spintronics. To ascertain the trend in the magnetic behavior and if it holds good with other test system, $\mathrm{Ni} / \mathrm{Al}$ and $\mathrm{Ni} / \mathrm{Cu}$ system is investigated. However, this revealed a different trend in magnetic behavior. This different magnetic property is attributed to their dissimilar interfacial bonding. As a matter of fact, the directional character of $N i-A l(d-p)$ hybridization forces $N i$ to have higher coordination number with $A l$ resulting in a decreasing but non-monotonic trend in $\mu_{a v}$. In $N i-C u$, the $s-d$ hybridization reduces the unoccupied $N i d$ down state leading to a decreasing trend in $\mu_{a v}$. This study strongly put forward the role of non magnetic spacer in 
modulating magnetic property but in a dissimilar fashion. In the next part of our study, the role of atomic scale structural heterogeneity at the magnetic/non-magnetic interface in modulating the IEC in $1 \mathrm{D} \mathrm{Fe} / \mathrm{Pt}$ nanowire is unraveled using first principles density functional approach. We also report an enhancement in the magnetic moment of the $\mathrm{Fe}$ atom at the $\mathrm{Fe} / \mathrm{Pt}$ interface, when compared to the magnetic moment of the corresponding $\mathrm{Fe}$ atom in the pristine Iron nanowire. Once again, a mechanism based on multi-step electron transfer and spin-flip is used to explain the increased magnetic moment of the interfacial $\mathrm{Fe}$ atom. The $J$ value as well as GMR is found to switch sign as the spacer width in the nanowire increases. Magnitude of $J$ value is found to decrease substantially for larger spacer width. The competition among short range and long range direct exchange, indirect exchange, and super exchange depending upon the spacer width is found to be responsible for the non-monotonous sign in $J$. Looking at the other multilayered structures with different combinations involving various spacer systems like, $\mathrm{Fe} / \mathrm{Pd}, \mathrm{Fe} / \mathrm{Cu}, \mathrm{Fe} / \mathrm{Ag}$ and $\mathrm{Fe} / \mathrm{Au}$, the IEC is studied in relation to specific spacer layer thickness. For the Fe/Pd nanowire system the magnetic properties as well as the switching behaviour of IEC is observed to be in the similar fashion to that of $\mathrm{Fe} / \mathrm{Pt}$ system. Where as the $\mathrm{Fe} / \mathrm{Cu}$ multilayered nanowire system behaves differently. 


\subsection{Future directions:}

Till now, our effort is to explain the magnetization of a spintronic device and it's controlled magnetic properties with non-magnetic spacer. From the series of studies we performed, it is evident that there is an advantage of multilayered nanowires structures in ultra high density magnetic memory devices. However in the currently used hard drive disk, the information is stored in the form of small magnetic domains with a particular direction of magnetization. In the process of writing data in the disk, when a pulse of current is applied to scan the disk, there is a magnetic field exists between pulse of current and manipulated magnetization causing waste of energy. To overcome these limitations, an alternative way is proposed to pass electric current directly through the nano-device to switch the magnetization direction, either by spin transfer from the spin polarized current or by induced field, instead of an external pulse current to generate the magnetic field. This unique feature is called spin torque effect and it can lead to development of ultra high density magnetic memory device which can run with low power consumption. Experimentally, it is already reported that this spin transfer torque can switch the direction of magnetization in multilayer pillars [87, 88]. It is also possible to push the domain wall (DW) (boundary of two different magnetic domains with different magnetization directions) along the length of the nanowire [89, 90, 91] which gives rise to a new type of domain wall based data storage device called racetrack memory device [92]. Since the currently used hard drive disks are two dimensional arrays of magnetic bits, it is reasonable to look for the three dimensional 
racetrack memory $(\mathrm{RM})$ device as an alternative approach to develop a much faster and cheaper ultra high density memory device. The RM constitute the information stored in magnetic domains being stacked in a tall column of magnetic material. Each domain has a positive (north) and a negative (south) pole and the bit length is decided by the spacing between consecutive DWs. Thus RM is basically a shift resister, in which the DWs i.e the bits can move to and fro along the reading and writing elements integrated in the system. Mostly the DWs can be read with magnetic tunnel junction (MTJ) magnetoresistive sensing device $[93]$, while the writing can be carried out using the spin momentum transfer torque effect [94, 95] from the current injected to racetrack from magnetic nano-elements. Also it can be achieved by using the fringing fields given by controlled motion of magnetic DW in a proximal nanowire.

The external uniform magnetic field can't be used to shift the series of DWs along the race track because the neighboring DW would start to move in opposite directions and will start to annihilate each other causing energy loss. Furthermore, the use of non-uniform local magnetic field to manipulate the DW is found to be expensive and inconvenient. On the other hand in RM, the current is allowed to pass through a magnetic material and gets spin polarized, because of spin dependent diffusive scattering, thus carries spin angular momentum. When this spin polarized current passes through the race track, the spin angular momentum of the current gets transferred to the DW applying a torque to the moment of the DW [96]. This results into the shift the DW along the race track. But the magnetic column should be sufficiently narrow so that the spin momentum transfer interaction of the 
current to the DW dominates over the self field of the current. It is rational to assume that the race tracks are needed to be composed of narrow magnetic nanowires. But the ability to set the DW polarity in a layered nanowire system by choosing appropriate current direction for a current pulse is yet to be explored. However, the most challenging aspect of the racetrack memory is the controlled and reliable motion of these DWs and the required pulse of spin polarized current density to move the DW along the race track. Further research is necessary to understand the current induced domain wall motion in multilayered nanowire including that of the Fe/Pt nanowire system. 


\section{Appendix A}

\section{Derivation of Thomas Fermi kinetic}

\section{energy}

The number of energy states between energy levels $E$ to $E+d E$ is:

$$
n_{g}=\frac{\pi}{4}\left(\frac{8 m l^{2}}{h^{2}}\right)^{\frac{3}{2}} \varepsilon^{\frac{1}{2}} \delta \varepsilon
$$

With $\varepsilon_{f}$ is the Fermi energy and all the states with energy smaller than $\varepsilon_{f}$ are the occupied state and contributes to the total energy of the system. Thus the total energy is the 
contributions from all the occupied states and can be presented as:

$$
\Delta E=2 \int_{0}^{\varepsilon_{f}} \varepsilon\left(\frac{8 m l^{2}}{h^{2}}\right)^{\frac{3}{2}} \varepsilon^{\frac{1}{2}} \delta \varepsilon
$$

The factor 2 comes as each energy state is doubly occupied with both spin-up and spindown electrons.

$$
\Delta E=\frac{8 \pi}{5}\left(\frac{2 m}{h^{2}}\right)^{\frac{3}{2}} l^{3} \varepsilon_{f}^{\frac{5}{2}}
$$

The Fermi energy is related to the total number of electrons is:

$$
\Delta N=2 \int n_{g}(\varepsilon) d \varepsilon
$$

using eqn.1.1 it becomes,

$$
\Delta N=\frac{8 \pi}{3}\left(\frac{2 m}{h^{2}}\right)^{\frac{3}{2}} l^{3}\left(\varepsilon_{f}\right)^{\frac{5}{2}}
$$

In terms of $\Delta N$ the eqn. 1.3 becomes:

$$
\Delta E=\frac{3}{5} \Delta N \varepsilon_{f}
$$




$$
\begin{array}{r}
\frac{3 h^{2}}{10 m}\left(\frac{3}{8 \pi}\right)^{\frac{2}{3}} l^{3}=\left(\frac{\Delta N}{l^{3}}\right)^{\frac{5}{3}} \\
\left(\frac{\Delta N}{l^{3}}\right)=n
\end{array}
$$

$n$ is the electron density. rearranging the above equation we get

$$
\Delta E=\frac{3}{10}\left(3 \pi^{2}\right)^{\frac{2}{3}}\left(\frac{h^{2}}{8 \pi^{2} m}\right) l^{3} \Delta N n^{\frac{5}{3}}
$$

in atomic unit

$$
\left(\frac{h^{2}}{8 \pi^{2} m}\right) l^{3} n^{\frac{5}{3}}=1
$$

kinetic energy density is

$$
t[n]=\frac{\Delta E}{l^{3}}=\frac{3}{10}\left(3 \pi^{2}\right)^{\frac{2}{3}} n(r)^{5} 3
$$

The total kinetic energy is

$$
T[n]=\int t[n] d r=\frac{3}{10}\left(3 \pi^{2}\right)^{2} 3 \int n(r)^{5} 3 d r
$$




$$
T[n]=C_{f} \int n(r)^{5} 3 d r
$$

The derivation of Tomas-Fermi energy gives an clear explanation of kinetic energy of the N-electron system. 


\section{Appendix B}

\section{$\mathrm{N}$ representability and $\mathrm{V}$}

\section{representability}

An electron density $n(r)$ is said to be V-representable if it corresponds to the ground state Hamiltonian with a suitable local potential $V(r)$. This potential $V(r)$ should be unique[86]. That means although a local potential $V(r)$ can give multiple ground state with different densities but two different potential can't give the same ground state density as represented in Figure. 2.1Even for a single particle system there are densities that don't correspond to ground state wave function.

But the N-representable condition can be satisfied for any reasonable density. The density 


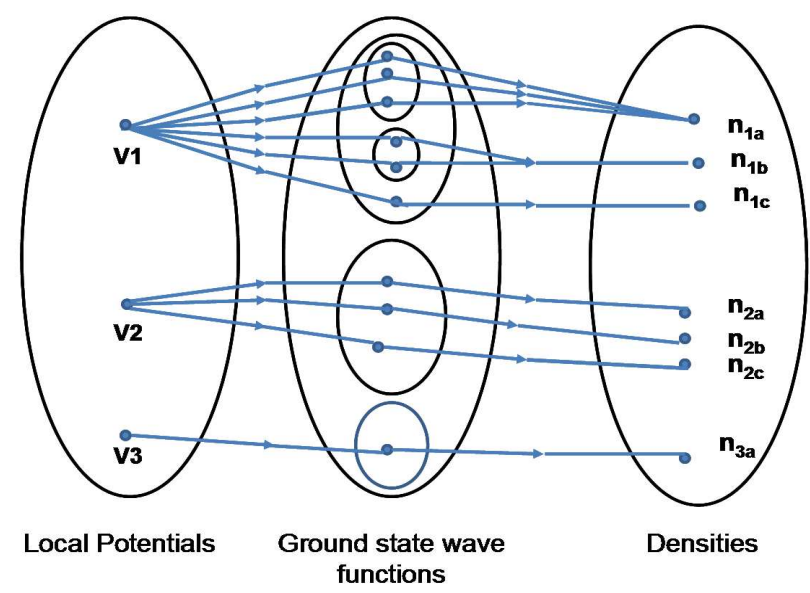

Figure 2.1: A mapping between potential and ground state density. Though the local potential can have multiple ground states, the two different local potential will never give the same ground state density.

is $\mathrm{N}$-representable if

$$
\begin{array}{r}
n(r) \geq 0 \text { nonumber } \\
\int n(r) d r=N \text { nonumber } \\
\int\left|\nabla n(r)^{\frac{1}{2}}\right|^{2} d r<\infty
\end{array}
$$

This gives a clear idea of $V$-representable and $N$-representable density. 


\section{Appendix C}

\section{Time Line}

All the calculated results presented in my thesis are performed on paticluster with the CPU using Dual Intel Xenon 2.8 GHz, Intel Pentium-4.3.0 GHz and Intel Xenon Quad Core 2.5 GHz with 2 GB RAM in each node.Here I have only used VASP code but with different pseudo-potentials and different set of grid point sampling of the Brillouin zone. Performance of different k-point sampling is presented in the Table. 3.1

Table 3.1: comparison between different k-point sampling.

\begin{tabular}{|c|c|c|c|c|c|}
\hline k-point & 111 & 113 & 115 & 116 & 117 \\
Total energy & 178.964 & 178.551 & 178.529 & 178.532 & 178.534 \\
CPU Time & 1907.00 & 660.31 & 930.18 & 772.39 & 1137.18 \\
\hline
\end{tabular}




\section{Appendix D}

\section{Copyrights}

The copyright permission for Figure. 1.3 is distributed under GFDL. GNU Free Documentation License(GFDL)

Version 1.2, February 2006

The copyright permission for Figure. 1.2 is distributed under GFDL.

GNU Free Documentation License(GFDL) Version 1.2, 9th October 2007

The copyright permission for Figure. 2.1] is distributed under GFDL.

GNU Free Documentation License(GFDL)

22nd November 2006

Permission is granted to copy, distribute and/or modify this document under the terms of the GNU Free Documentation License, Version 1.2 or any later version published by the Fre e Software Foundation; with no Invariant Sections, no Front-Cover Texts, and no Back- 
Cover Texts.

A copy of the license is included in the section entitled "GNU Free Documentation License". GNU Free Documentation License(GFDL)

Author: Wolfram Quester: I, the copyright holder of this work, hereby release it into the public domain. This applies worldwide. In case this is not legally possible,I grant any entity the right to use this work for any purpose, without any conditions, unless such conditions are required by law A guide line to follow public domain is described in the section entitled "Wikipedia:Public domain"

The GNU Free Documentation License (GNU FDL or simply GFDL) is a copyleft license for free documentation, designed by the Free Software Foundation (FSF) for the GNU Project. It is similar to the GNU General Public License, giving readers the rights to copy, redistribute and modify a work and requires all copies and derivatives to be available under the same license. Copies may also be sold commercially, but, if produced in larger quantities (greater than 100), the original document or source code must be made available to the work's recipient. The GFDL was designed for manuals, textbooks, other reference and instructional materials, and documentation which often accompanies GNU software. However, it can be used for any text-based work, regardless of subject matter. For example, the free online encyclopedia Wikipedia uses the GFDL for all of its text. Wikipedia:Public domain For all practical purposes on Wikipedia, the public domain comprises copyrightfree works: anyone can use them in any way and for any purpose. Proper attribution to the author or source of a work, even if it is in the public domain, is still required to avoid 
plagiarism. The public domain is generally defined (e.g. by the U.S. Copyright Office) as the sum of works that are not copyrighted, i.e. * that were not eligible for copyright in the first place, or * whose copyright has expired. However, there is no such thing as the public domain on the Internet. International treaties, like the Berne Convention, are not self-executing and do not supersede local law. There is no globally valid "International Copyright Law" that would take precedence over local laws. Instead, signatory countries of the Berne Convention have adapted their laws to comply with the minimum standards set forth by the treaty, often with stronger provisions than required. Whether or not something is copyright-free in some country depends on the laws of individual countries. Wikipedia, and the Wikimedia Foundation, its legal body, are based in Florida, United States. Although legislation is sometimes unclear about which laws are to apply on the Internet, the primary law relevant for Wikipedia is that of the United States. For re-users of Wikipedia content, it is the laws of their respective countries. In the U.S., any work published before January 1, 1923 anywhere in the world[1] is in the public domain. Other countries are not bound to that 1923 date, though. Complications arise when special cases are considered, such as trying to determine whether a work published later might be in the public domain in the U.S., or when dealing with unpublished works. When a work has not been published in the U.S. but in some other country, that other country's copyright laws also must be taken into account. Re-users of Wikipedia content also might find the explanations here useful.

The copyright permission for Figure. 3.18, Figure. 3.19, Figure. 3.20, Figure. 3.20 is taken from APS and attached as a figure in Figure.4.1. 


\section{APS physics}

April 9, 2009

Puspamitra Panigrahi

Michigan Tech Univ

1914 A Woodmar Dr

Houghton, MI 49931 USA

\section{$\underline{\text { Ref \# } 7549}$}

Thank you for your permission request dated on April 6, 2009. We are pleased to grant you a non-exclusive, nontransferable permission, English rights, limited to print and electronic format, provided you meet the criteria outlined below. Permission is for a one-time use and does not include permission for future editions, updates, databases, translations, or any other matters. Permission must be sought for each additional use. This permission does not include the right to modify APS material.

Please print the required copyright credit line on the first page that the material appears: "Reprinted (abstract/excerpt/figure) with permission from [FULL REFERENCE CITATION] as follows: authors names, journal title, volume number, page number and year of publication. Copyright (YEAR) by the American Physical Society.

The following language must appear somewhere on the website: "Readers may view, browse, and/or download material for temporary copying purposes only, provided these uses are for noncommercial personal purposes. Except as provided by law, this material may not be further reproduced, distributed, transmitted, modified, adapted, performed, displayed, published, or sold in whole or part, without prior written permission from the American Physical Society."

Provide a hyperlink from the reprinted APS material (the hyperlink may be embedded in the copyright credit line). APS's link manager technology makes it convenient and easy to provide links to individual articles in APS journals. For information, see: http://publish.aps.org/linkfaq.html

You must also obtain permission from at least one of the authors for each separate work, if you haven't done so already. The author's name and address can be found on the first page of the published Article.

Use of the APS material must not imply any endorsement by the American Physical Society.

Permission is granted for use of the following APS material only: Fig. 2a,2b,3a,3b, Phys Rev. B, Vol. 77, 144430(2008)

Permission is limited to the single title specified or single edition of the publication as follows:

A PhD thesis entitled "Controlling magnetic Properties of Ultra narrow multilayered nanowires" to be published by Michigan Tech. University.

If you have any questions, please refer to the Copyright FAQ at: http://forms.aps.org/author/copyfaq.html or contact me at assocpub@aps.org.

Sincerely,

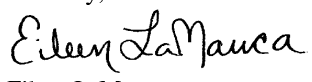

Eileen LaManca

Publications Marketing Coordinator

Figure 4.1: The photo copy of the permission letter showing permission is granted for the figures.(Reprinted from, Partha.Pratim.Pal, Ranjit.Pati, Phys. Rev. B. 77, 144430(2008), (C)(2008) The American Physical society.) 


\section{References}

[1] P. Grünberg, R.Schreiber, Y.Pang, M.B.Brodsky, H.Sowers, Phys. Rev. Lett. 57, 2442 (1986).

[2] M. N. Baibich, J.M.Broto, A.Fert, F.N.Dau, F.Petroff, P.Eitenne, G.Creuzet, A.Friederich, J.Chazelas, Phys. Rev. Lett. 61, 2472 (1988).

[3] G. Binasch, P.Grünberg,F.Saurenbach, W.Zinn, Phys. Rev. B 39, 4828 (1988).

[4] S.S.Parkin, N.More, K.P.Roche, Phys. Rev. Lett. 64, 2304 (1990).

[5] S.S.Parkin, R.Bhadra, K.P.Roche, Phys. Rev. Lett. 66, 2152 (1991).

[6] S.T.Purcell, W.Flokerts, M.T.Jhonson, N.W.E.McGee, K.Jagar, J.Stegge, W.B.Zeper, W.Hoving, P.Grünberg, Phys. Rev. Lett. 67, 903 (1991).

[7] P.Bruno, C.Chapet, Phys. Rev. Lett. 67, 1602 (1991).

[8] P.Bruno, C.Chapet, Phys. Rev. B. 46, 261 (1992).

[9] P.Lang, L.Nordstrom, R.Zeller, P.H.Dederichs, Phys. Rev. Lett. 71, 1927 (1993). 
[10] R.Hafner, D.Spisak, R.Lorenz, J.Hafner, J.Phys. Condensed.Matter 14, 4297 (2002).

[11] J.Opitz, P.Zahn, J.Binder, I.Mertig, Phys. Rev. B. 63, 094418 (2001).

[12] J.Kudrnovsky, V.Drchal, I.Turek, P.Bruno, P.Weinberger, J. Phys. Condensed. Matter 13, 8539 (2001).

[13] A.Yoshihara, J.T.Wang, K.Takanashi, K.Himi, H.Pujimori, P.Grunberg, Phys. Rev. B. 63, 100405(2001).

[14] B.Heinrich, Z.Celinski, J.F.Cochran, W.B.Muir, J.Rudd, Q.M.Zhong, A.S.Arrott, K.Myrtle, J.Kirschner, Phys. Rev. Lett. 64, 673(1990).

[15] J.E.Mattson, C.H.Sowers, A.Berger, S.D.Bader, Phys. Rev. Lett. 68, 3252(1992).

[16] A.Blondel, J.P.Meier, B.Doudin, J.P.Ansermet, App. Phys. Lett. 65, 3019(1994).

[17] L.Piraux, J.M George, J.F.Despres, C.Leroy, E.Ferain, R.Legras, K.Ounadjela, A.Fert, App. Phys. Lett. 65, 2484(1994).

[18] M.Tanase, D.M.Silevitch, A.Hultgren, L.A.Bauer, P.C.Searson, G.J.Mayer, D.H.Reich, J. App. Phys. 91, 8549(2002).

[19] J.A.Katine, A.Palanisami, R.A.Bhrumn, App. Phys. Lett. 74, 1883(1999).

[20] K.Liu, K.Nagodawithana, P.C.Searson, C.L.Chien, Phys. Rev. B. Rapid Comm 74, 1883(1999). 
[21] C.L.Chien, L.Sun, M.Tanase, L.A.Bauer, A.Hultgren, D.M.Silevitch, G.J.Mayer, P.C.Searson, D.H.Reich, Magn. Magn. Matter. 249, 146(2002).

[22] H.S.Park, Nano. Lett. 6, 958(2006).

[23] F.Elhoussine, L.Vila, L.Piraux, G.Faini, Magn. Magn. Matter. 290, 116(2005).

[24] S.Urazhdin, W.P.Pratt, J.Bass, Magn. Magn. Matter. 282, 264(2004).

[25] C.Z.Wang, G.W.Meng, Q.Q.Fang, X.S.Peng, Y.W.Wang, Q.Fang, L.Zhang, J. Phys. D 35, 738(2002).

[26] S.D.Willoughby, J.M Maclern, T.Ohkubo, S.Jeong, M.McHenry, D.E.Laughlin, S.Choi, S.Kwon, J. App. Phys. 91, 8822(2002).

[27] Y.Wang, G.M.Stocks, A.Rusanu, D.M.C.Nicholson, M.Eisenbach, Q.Zhnag, J.P.Liu, IEEE. Trans. Magn. 43, 3103(2002).

[28] Zs.Major, S.B.Dugdale, T.Jarlborg, E.Bruno, B.Ginatempo, J.B.Stauunton, J.Poulter, J. Phys. Condensed. Matter 15, 3619(2003).

[29] S.Z.Chu, S.Inoue, K.Wade, Y.Kanke, K.Kurashima, J. Electrochem. Soc. 152, 42(2005).

[30] Y.Hou, H.Kondoh, R.Che, M.Takeguchi, T.Ohta, small. 238, 2(2005).

[31] J.Gao, Q.Zhan, W.He, D.Sun, Z.Cheg, App. Phys. Lett. 86, 230506(1999).

[32] Y.H.Huang, H.Okumura, G.C.Hadjipanayis, D.Weller, J. App. Phys. 91, 8549(2002). 
[33] J.Choi, S.J.Oh, H.Ju, J.Choen, Nano. Lett. 5, 2179(2005).

[34] Y.Peng, T.Cullis, G.Mobus, X.Xu, B.Inkson, Nanotech. 18, 485704(2007).

[35] R.G.Par and W.Yang, Density-Functional Theory of Atoms and Molecules. (Oxford Science Publication).

[36] M.Onink, Scripta Metall. Mater. 29, 1011(1993).

[37] C.Kittel, Introduction to solid state Physics.

[38] G.Kresse and J.Furthüller, Phys. Rev. B 54, 11169(1996).

[39] Vienna ab initio simulation package (Technische Universität Wien, 1999).

[40] E.Schrödinger, Ann. Physik. 79, 361( 1926).

[41] M.Born and R.Oppenheimer, Ann. Phys. 84, 457(1927).

[42] J.C.Slater, Phys. Rev. 81, 385(1951)

[43] D.R.Hartree, Proc. R. Soc. London. .A 113, 621(1928).

[44] V.Fock, Z. Phys. 61, 126(1930).

[45] R.O.Jones and O.Gunnarsson Rev. Mod. Phys. 61, 689(1989).

[46] Density Functional Theory of Atoms and Molecules. Oxford University Press, New York, 1989.

[47] W.Kohn, A.D.Becke, R.G.Parr, 100, 12974(1996). 
[48] R.M.Martin, Electronic Structure. (Cambridge University Press)

[49] L.H.Thomas, Proc. Cambridge Philos. Soc. 23, 542(1927).

[50] P.Hohenberg and W.Kohn, Phys. Rev. B. 136, 864(1964).

[51] M.Levy, Phys. Rev. A. 26, 1200(1982).

[52] L.D.Site, J.Phys. A. Math. Theor. 40, 2787(2007).

[53] W.kohn, L.J.Sham, Phys. Rev. 140, 1133(1965).

[54] J.Kohanhoff, Electronic Structure Calculations for solids and molecules. (Cambridge University Press).

[55] N.H. March, Electron Density Theory of Atoms and Molecules (Academic Press, 1992), ISBN 0-12-470525-1.

[56] N.W.Ashcroft, and N.D.Mermin, solid state Physics. Harcourt College Publishers, (1997).

[57] D.J.Chadi,and M.L.Cohen, Phys. Rev. B. 8, 5747(1973).

[58] H.J.Monkhorst, and J.D.Pack, Phys. Rev. B. 13, 5188(1976).

[59] J.C.Phillips, Phys. Rev. 112, 685(1958).

[60] J.C.Phillips, and L.Kleinman, Phys. Rev. 116, 287(1959).

[61] M.L.Cohen, and V.Heine, Phys. Rev. 24, 37(1970). 
[62] M.Podgorny, Phys. Rev. B. 43, 11300(1991).

[63] P.T.Jochym, K.Parlinski, A.M.Oles, Euro. Phys. B. 61, 173(2008).

[64] P.P.Pal, R.Pati, Phys. Rev. B. 77, 144430(2008).

[65] D.Repetto, T.Y.Lee, S.Rusponi, J.Honloka, K.Kuhnke, V.Sessi, U.Starke, H.Brune, P.Gambardella, C.Carbone, A.Enders, K.Kern, Phys. Rev. B. 74, 054408(2006).

[66] B.Stahl, N.S.Gajbhiye, G.Wilde, D.Kramer, J.Ellrich, M.

[67] P.Panigrahi, R.Pati, Phys. Rev. B. 76, 024431(2007).

[68] A.Delin, E.Tosatti, R. Weht, Phys. Rev. Lett. 92, 057201 (2004).

[69] B.Y.Yavorsky and I.Mertig, Phys. Rev. B. 74, 174402 (2006).

[70] J.C.Tung, G.Y.Guo, Phys. Rev. B. 76, 094413 (2007).

[71] K.M.Smelova, D.I.Bazhanov, V.S.Stepanyuk, W.Hergert, A.M.Saletsky, P.Bruno, Phys. Rev. B. 77, 033408 (2008).

[72] M.T.Johnson, S.T. Purcell, N.W.E.McGee, R.Coehoorn, J.Stegge, W.Hoving, Phys. Rev. Lett. 68, 2688 (1992).

[73] P.J.H.Bloemen, M.T.Johnson, M.T.H. van de Vorst, R.Coehoorn, J.J.de Vries, R.Jungbult, J.Stegge, A.Reinders, W.J.M.de Jonge, Phys. Rev. Lett. 72, 764 (1994).

[74] J.J.de Vries, A.A.P.Schudelaro, R.jungbult, P.J.H.Bloemen, A.Reinders, J.Kohlhepp, R.Coehoorn, W.J.M.de Jonge, Phys. Rev. Lett. 75, 4306 (1995). 
[75] J.Barnas, J.Magn. Magn. Mater. 111, 215 (1992).

[76] O.O.Brovko, P.A.Ignatiev, V.S.Stepanyuk, P.Bruno, Phys. Rev. Lett. 101, 036809 (2008).

[77] M.Julliere, Phys. Lett. 54A, 225 (1975).

[78] P.H.Dederichs, K.Wildberger, and R.Zeller, Physica. B. 237, 239 (1997).

[79] J.E.Ortega, and H.J.Himpsel, Phys. Rev. Lett. 69, 844 (1992).

[80] A.T.Costa, Jr., J.Albuquerque, R.B.Muniz, Phys. Rev. B. 5613697 (1997).

[81] P.Panigrahi, R.Pati, Phys. Rev. B. 79, 014411(2009).

[82] H.A.Kramers, Physica 1182 (1934).

[83] P.W. Anderson, Phys. Rev. 79350 (1950).

[84] J.B.Goodenough, Magnetism and the Chemical Bond, Wiley-Interscience, New York (1963).

[85] J.Kanamori, J. Phys. Chem. Solids 1087 (1959).

[86] G.F.Giuliani, and G.Vignale, Quantum Theory of Electron Liquid.(Cambridge University Press).

[87] K.Bussmann, G.A.Prinz, S.F.Cheng, D.Wand, App. Phys. Lett. 75, 2476 (1999).

[88] J.A.Katine, F.J.Albert, R.A.Buhrman, E.B.Myers, and D.C.Ralph, Phys. Rev. Lett. 101, 3149 (2000). 
[89] A.Yamaguchi, T.Ono, S.Nasu, K.Miyake, K.Mibu, T.Shinjo, Phys. Rev. Lett. 92, 077205 (2004).

[90] M.Kläui, J. Phys. Condens. Matter 20, 313001 (2008).

[91] M.Kläui, P-O.Jubert, R.Allenspach, A.Bischof, J.A.C.Bland, G.Faini, U.Rüdiger, C.A.F.Vaz, L.Vila, and C.Vouille, Phys. Rev. Lett. 95, 026601 (2005).

[92] S.S.S.Parkin, M.Hayashi, and L.Thomas, Science 320, 190 (2008).

[93] S.S.S.Parkin, Proc. IEEE 91, 661 (2003).

[94] J.Slonczewski, J.Magn. Magn. Mater. 159, L1 (1996).

[95] L.Berger, Phys. Rev. B. 54, 9353 (1996).

[96] A.Vanhaverbeke, A.Bischof, and R.Allenspach, Phys. Rev. Lett. 101, 107202 (2008). 